University of Louisville

ThinkIR: The University of Louisville's Institutional Repository

Electronic Theses and Dissertations

$12-2013$

\title{
Charge collection and surface recombination effects in organic bulk heterojunction solar cells.
}

Buddika KA. Abeyweera 1980-

University of Louisville

Follow this and additional works at: https://ir.library.louisville.edu/etd

Part of the Electrical and Computer Engineering Commons

\section{Recommended Citation}

Abeyweera, Buddika KA. 1980-, "Charge collection and surface recombination effects in organic bulk heterojunction solar cells." (2013). Electronic Theses and Dissertations. Paper 7.

https://doi.org/10.18297/etd/7

This Doctoral Dissertation is brought to you for free and open access by ThinkIR: The University of Louisville's Institutional Repository. It has been accepted for inclusion in Electronic Theses and Dissertations by an authorized administrator of ThinkIR: The University of Louisville's Institutional Repository. This title appears here courtesy of the author, who has retained all other copyrights. For more information, please contact thinkir@louisville.edu. 


\title{
CHARGE COLLECTION AND SURFACE RECOMBINATION EFFECTS IN ORGANIC BULK HETEROJUNCTION SOLAR CELLS
}

\author{
By \\ Buddika K. Abeyweera \\ B.Sc., University of Peradeniya, 2006 \\ M.S., University of Louisville, 2009

\begin{abstract}
A Dissertation
Submitted to the Faculty of the

J.B.Speed School of Engineering at the University of Louisville in Partial Fulfillment of the Requirements for the Degree of
\end{abstract} \\ Doctor of Philosophy \\ Department of Electrical \& Computer Engineering \\ University of Louisville \\ Louisville, KY 40292
}

December 2013 
Copyright 2013 by Buddika K. Abeyweera

All Rights Reserved 



\title{
CHARGE COLLECTION AND SURFACE RECOMBINATION EFFECTS IN ORGANIC BULK HETEROJUNCTION SOLAR CELLS
}

\author{
By \\ Buddika K. Abeyweera \\ BSc., University of Peradeniya, 2006 \\ MS., University of Louisville, 2009
}

A Dissertation approved on

December 05, 2013

By the following Thesis Committee:

$\overline{\text { Dr. Bruce Alphenaar (Thesis Director) }}$

Dr. Gamini Sumanasekera

Dr. Kevin Walsh

Dr. Sergio Mendes

Dr. Shamus McNamara 


\section{DEDICATION}

This dissertation is dedicated to my parents and to my family 


\section{ACKNOWLEDGMENTS}

I thank everyone who have supported me and helped me along the way to complete this dissertation. I am most grateful to my advisor, Dr. Bruce Alphenaar for all his support and patience. His unique ideas helped me develop some of the most interesting experiments that got to work on. I believe, the skills that I have learnt from him have largely contributed to make me a better researcher. I would also like to thank Dr. Gamini Sumanasekera for all his support since my master's degree. Research exposure I gained from my master's thesis helped me a lot to continue my Ph.D. research. Then I would like to thank Dr. Shamus McNamara for the useful discussions that we had over the years. His classes on Semiconductor principles proved to be vitally important for my research. I thank Dr. Sergio Mendes for his useful insights on my research. His ideas in experimental optics have helped me to improve my work. I thank Dr. Kevin Walsh for his useful evaluation of my dissertation and for being a part of my committee. The cleanroom, which functions under his administration at the University of Louisville, helped me a lot to continue my research.

I would like to thank the current and the former post docs, research engineers and students whom I had the pleasure to work with. Dr. Shavil Desai, Dr. Tanesh Bansal and Dr. Lin (George) helped me a lot with equipment training, techniques and related issues at the beginning of my research. I thank my lab mates for useful discussions we had over the years that helped me to improve and better understand my work. In the past 
few years, I have worked with almost every staff member in Schumaker Research Building and the cleanroom of the University of Louisville. I thank all of them for their help and guidance.

Lastly, I would like to thank my parents for their encouragement and support. I would not have gone this far in my education if it was not for their encouragement which has been generously given to me since my childhood. I am so thankful to my wife, Piyumika, for all her love, patience, encouragement and unwavering support given to me throughout these years, while successfully pursuing her own career goals, and fulfilling the responsibilities of Motherhood. I am so grateful to my son, Tharul, who is too young to understand what "thatta" is doing. He has been a constant source of joy and motivation. My family deserves most of the credit of this work. 


\section{ABSTRACT \\ CHARGE COLLECTION AND SURFACE RECOMBINATION EFFECTS IN \\ ORGANIC BULK HETEROJUNCTION \\ SOLAR CELLS}

\section{Buddika K. Abeyweera \\ December $05^{\text {th }}, 2013$}

Charge transfer and charge extraction mechanisms are two prevalent issues in the growing field of organic solar cells. Due to their complexity in nature, new methods need to be involved in addressing the fundamental properties associated with organic polymer solar cells. This dissertation has focused on developing a new method to estimate the charge collection lengths and surface recombination lengths of organic polymer solar cells.

Photocurrent spectra have been analyzed systematically to observe the dependence on thickness of the active material. A red shift of the peak of the normalized photocurrent with respect to the device thickness has been further analyzed for two major material systems used in organic polymer solar cells, namely MDMO-PPV: PCBM and P3HT: PCBM. A theoretical model that measures the charge extraction of bulk hetero junction solar cell structures has been used taking into account of three main parameters including charge carrier collection length, absorption variation and surface 
recombination. This model has led to estimate two important parameters associated with charge transfer, recombination and extraction of organic solar cells which will provide opportunities for improvements in the performance of organic electronic devices. Key results are summarized as follows.

A complete analysis of photocurrent spectra has been done to see its variation with active material thickness of well-known two material systems of bulk heterojunction organic solar cells. Results of these preliminary measurements suggest that peak of the photocurrent for both systems red shift with increasing thickness. Charge extraction model is introduced to explain the initial red shift of the photocurrent. This model fits well with the experimental results. Further analysis of the model suggests that the charge collection lengths can be estimated for organic polymer structures. Theoretical model gives higher collections lengths for MDMO-PPV solar cells while a lower collection length for P3HT solar cells.

This model also has the capability to estimate the surface recombination length of organic bulk heterojunction solar cells. Different interfacial layers have been used to fit to the model calculation. These results suggest that the least surface recombination lengths were achieved with solar cells of PEDOT-PSS. This method can be used to optimize the interfacial layers to improve the efficiency in organic solar cells.

AC photocurrent measurements have been carried out to observe the frequency dependence of organic solar cells. Main results show that increasing response time from the light source increases the performance of the solar cells. Further analysis of these 
results suggests that thicker devices of organic polymer solar cells can be applied with longer response time light sources.

Degradation of organic polymer solar cells over a period of several days has been carried out to observe the stability of solar cells with device thickness. Results suggest that the degradation occurs regardless of device thickness of the active material. A new probing technique called capacitive photocurrent spectroscopy (CPS) has been carried out with P3HT solar cells to observe the charge dissociation efficiency. The CPS measurements suggest that sub band gap states have higher dissociation efficiencies that may not contribute to overall photocurrent of the solar cells. 


\section{TABLE OF CONTENTS}

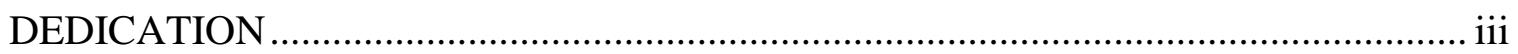

ACKNOWLEDGMENTS _...................................................................................... iv

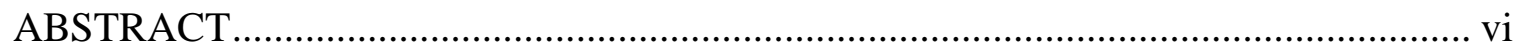

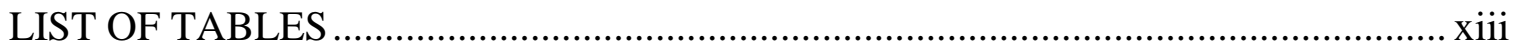

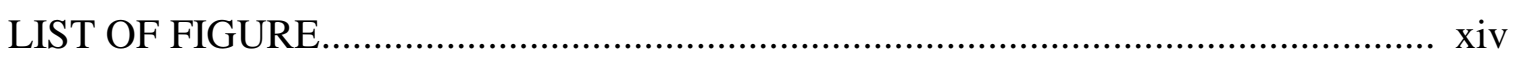

$\begin{array}{ll}\text { CHAPTERS } & \text { PAGE }\end{array}$

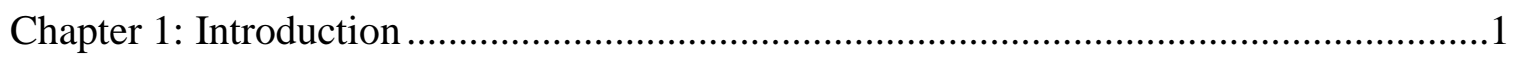

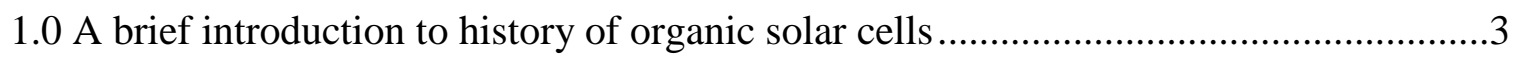

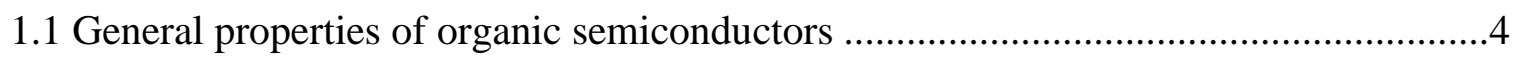

1.2 Photogeneration mechanism in organic polymer solar cells......................................

1.3 Materials used in fabricating organic solar cells......................................................12

1.4 Recombination losses in organic solar cells ……...................................................14

1.5 Mobility of the charge carriers in the organic solar cells..........................................15

1.5.1 Dark Injection (DI) Technique...............................................................16

1.5.2 Space charge limited current-voltage measurement ...................................16 
1.5.3 Time of Flight Technique (TOF) …………………................................16

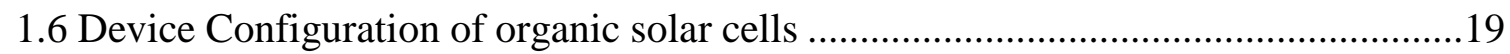

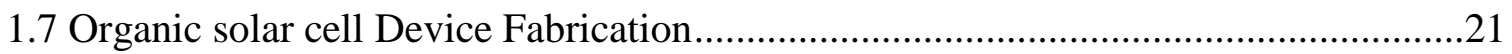

Chapter 2: Initial characterization of organic solar cells ...............................................26

2.1 Optical excitation sources used in characterization of the solar cells.........................26

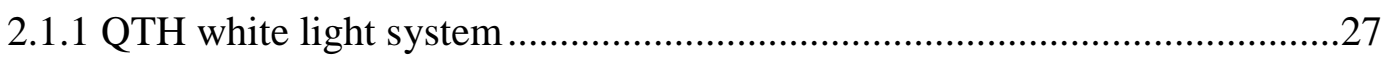

2.1.2 Spectra Physics ultra-fast pulse laser system............................................28

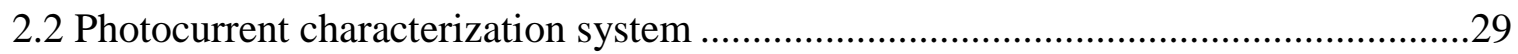

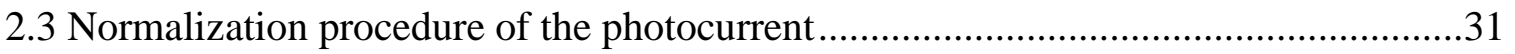

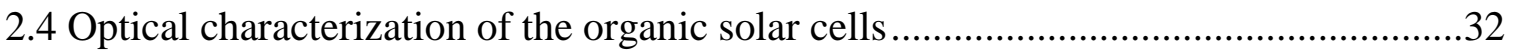

2.4.1 Atomic force microscopy measurements of organic solar cells ....................32

2.4.2 Absorbance measurements of organic solar cells .........................................33

2.5 Photoluminescence spectra of organic solar cells .........................................................37

$2.6 \mathrm{~J}-\mathrm{V}$ characterization of organic solar cells ..................................................................38

2.7 Mobility measurements of P3HT: PCBM solar cells (Time of flight technique) .......40

2.8 Initial photocurrent measurements on organic blends ...............................................4 
Chapter 3: Charge extraction model

3.1 Photoinduced charge generation in semiconductors 48

3.2 Derivation of the charge extraction model .54

3.2.1 Charge collections in model derivation for front illumination .54

3.2.1.1 Dependence of Charge extraction on absorption coefficient for P3HT and MDMO-PPV solar cells .57

3.2.1.2 Charge collection dependence on absorption coefficient and thickness .59

3.2.1.3 Charge collections spectra for P3HT and MDMO-PPV samples..61

3.2.1.4 Absorption coefficient dependence on thickness for different charge collection lengths .63

3.2.1.5 Peak absorption coefficient dependence on thickness for different mobilities. .64

3.2.2 Derivation of the model for back illumination .65

3.3. Front/Back Illumination effect on charge collection .67 
3.4 Charge collection dependence on surface recombination in P3HT samples

Chapter 4: Experimental photocurrent results

4.1 Introduction .71

4.2. Effect of interfacial layers on charge collection efficiency

4.3. Photocurrent dependence on thickness for P3HT and MDMO-PPV solar cells

4.4 Charge collection dependence on composition of P3HT: PCBM solar cells .82

4.5 Charge collection variation in front/back illumination .84

Chapter 5: AC photocurrent spectroscopy of organic solar cells .88

5.1 AC photocurrent spectroscopy of organic solar cells .87

5.2 Degradation measurements of P3HT: PCBM solar cells .92

5.3 Capacitive photocurrent Spectroscopy .94

Chapter 6: Conclusions and Discussion. .99

Future work 103

References .105

Curriculum Vitae .114 


\section{TABLE OF CONTENTS}

TABLE

PAGE

2.1 Summary of J-V characteristics of MDMO-PPV and P3HT organic solar cells.........40

2.2 The summary of mobilities of P3HT: PCBM samples for different weight ratios ......44 


\section{LIST OF FIGURES}

FIGURE

PAGE

1.0 Evolvement of the Efficiency of the solar cells from 1975 to 2010 ............................

$1.1 \pi$-bond formation in $\mathrm{sp}^{2}$-hybridized carbon atoms in simplest conjugation system ......5

1.2 Monomer structures of the conjugated polymers used in this work .........................6

1.3 Monomer structure of the fullerene derivative used in organic solar cells ..................

1.4 Band alignment of polymer (p type) and PCBM (n type) ....................................10

1.5 Band alignment of MDMO-PPV: PCBM (left) and P3HT: PCBM (right) systems....12

1.6 Experimental setup used in time of flight (TOF) measurement .............................18

1.7 Transient currents using TOF measurements .........................................................19

1.8 Device configuration of single layer and bilayer devices .................................... 20

1.9 Device configuration of bulk hetero junction-BHJ and bilayer devices....................21

1.10 Flow of the fabrication of layers in organic bulk heterojunction solar cells ............24

1.11 Basic device configuration of the organic solar cell .........................................25 
2.1 Spectral photon flux of the sun

2.2 Spectral irradiance of QTH white light source measured by Silicon photo detector...28

2.3 Schematic setup used to measure the photocurrent spectra of organic solar cells.......29

2.4 No. of photons in visible spectrum by the light absorption for the QTH light source.32

2.5 Atomic force microscopy pictures of P3HT: PCBM organic solar cells

2.6 Absorption coefficient spectrum of P3HT: PCBM

2.7 Absorption coefficient spectrum of MDMO-PPV: PCBM .35

2.8 Absorbance spectrum of MDMO-PPV: PCBM at different temperatures .36

2.9 Photoluminescence spectra of MDMO-PPV: PCBM and P3HT: PCBM .37

2.10 J-V characteristic of thickness of 100nm P3HT: PCBM solar cell .38

$2.11 \mathrm{~J}-\mathrm{V}$ characteristic of thickness of $100 \mathrm{~nm}$ and $800 \mathrm{~nm}$ of P3HT: PCBM solar cells...39

2.12 The transient current measurements for P3HT: PCBM sample of 5:4 wt $\%$ .42

2.13 The transient measurements for P3HT: PCBM sample different compositions. .43

2.14 Absorption percentage of P3HT: PCBM and MDMO-PPV: PCBM .45

2.15 Photocurrent vs. thickness dependence of P3HT: PCBM organic solar cell .46

3.1 Charge generation at a distance $\mathrm{x}$ from the front .47 
3.2 Attenuation of light in a slab as a function of absorption coefficient

3.3 Decay of charge generated along the distance of the active layer .50

3.4 Diffusion and Drift in a semiconductor medium .51

3.5 Drift and Diffusion components of decay per unit charge. .53

3.6 Schematic of the charge collection lengths for electrons and holes .55

3.7 Generation of population of charge $\mathrm{Q}(\mathrm{x})$ at a distance $\mathrm{dx}$ from the front contact.......56

3.8 Fraction of extracted charge as a function of the abs. coeff. for thicknesses .58

3.9 The charge collection plotted as a function of abs. coeff. for different thicknesses....59

3.10 Abs. coeff. of peak positions of P3HT as a function of device thickness. .60

3.11 Fraction of charge collected spectra different thickness of P3HT and MDMO

3.12 Abs. coeff. for charge peak for various collection lengths for P3HT .63

3.13 Peak abs. coeff. for charge peak for $d_{e}>d_{h}, d_{e}<d_{h}$ and $d_{e}=d_{h}$ of P3HT. .64

3.14: Generation of charge $Q(X)$ at a distance $d x$ from the front contact .65

3.15 Abs. coeff. at extracted charge peak for the front/back illumination in P3HT .68

3.16 Abs. coeff. measured at charge peak for different recombinations in P3HT .70

4.1 Absorption and Photoluminescence spectra of MDMO and P3HT solar cells .72 
4.2 J-V curves of P3HT solar cells with three different interfacial conditions .74

4.3 Norm. Photocurrent spectra for different thicknesses of interfacial layers .75

4.4 Abs. coeff. measured at photocurrent charge peak for different interfacial layers......76

4.5 Norm. Photocurrent spectra for different active layer thickness of MDMO-PPV ......77

4.6 Norm. Photocurrent spectra for four different active layer thickness of P3HT. .78

4.7 Abs. coeff. for peak positions of MDMO-PPV as a function of device thickness .......80

4.8 Abs. coeff. for peak positions of P3HT as a function of device thickness

4.9 Peak abs. coeff. as a function of thickness of P3HT for different weight ratios .82

4.10 Abs. coeff. at extracted photocurrent peak for front/back illumination in P3HT

4.11 Electron mobility as a function of PCBM\% .85

5.1 The schematic diagram of the AC photocurrent setup

5.2 The AC photovoltage for P3HT: PCBM 5:4 wt\% samples for different frequencies .90

5.3 Norm. photovoltage peak for P3HT 5:4 wt \% samples for different frequencies .91

5.4 The frequency for photovoltage peak vs. thickness for P3HT samples of wt $\%$ ratios 92

5.5 Norm. Integrated photocurrent versus device thickness for P3HT solar cells .93

5.6 Degradation of P3HT solar cells for different thicknesses for seven days .94 
5.7 Schematic design of capacitive photocurrent spectroscopy...

5.8 Capacitive photocurrent spectroscopy and abs. measurement of P3HT solar cells.....97 


\section{CHAPTER I: INTRODUCTION}

Organic solar cells have attracted much attention among researchers in past few decades due to many reasons. Ease of fabrication, mechanical flexibility and low cost are major advantages of organic solar cells over conventional photovoltaics. However, the main hurdle for further development of organic solar cells is the low efficiency associated with it. The highest efficiency the organic solar cells have achieved so far is about $10 \%$ with multi-layer devices. The efficiencies are still improving due to the introduction of new materials and engineering of more sophisticated device structures. Even though the efficiencies of organic Photovoltaics have not yet reached those of the inorganic counterparts $(\sim 40 \%)$, the technologies derived from organic photovoltaics such as light emitting diodes have recently reached the commercial market.

The highest efficiencies of current organic solar cells have shown by tandem and Bulk Hetero-junction (BHJ) device configurations, in which the donor-acceptor material are mixed to enhance the interfacial area to capture more free charge carriers for electricity generation[1]. Unlike p-n junction solar cells, organic solar cells create electron-hole pairs (EHPs) upon light absorption which are bound to each other. In order to create a barrier-free electron (or hole) flow across an external load, these e-h pairs have to transport to an interface, dissociate and finally collected by the electrodes. A detailed explanation of these excited carriers will be discussed in later sections. Even though there are many articles published about the above-mentioned phenomena, there is lack of 
understanding on how exactly the carriers are generated in organic solar cells, and the mechanisms of e-h pairs separation and transportation to the electrodes. To improve the overall efficiencies of these types of thin-film solar cells, one has to understand a comprehensive mechanism of this technology.

This dissertation work focuses on the photocurrent measurements of bulk heterojunction (BHJ) solar cells with different active layer thicknesses. It is observed that the main photocurrent peak that corresponds to conjugated polymer shifts towards higher wavelengths when the thickness of the active layer is increased. The optical absorption of these devices is matched with the photocurrent peak for thin active layer devices. This behavior is majorly due to time variations of electron and holes charge transport lengths and surface recombination effect that reduces the high energy excitons when the device thickness is increased.

The last chapters will discuss the photocurrent analysis experiments with AC light, to observe this variation of photocurrent peak in thick devices. In addition, the capacitive photocurrent technique will be discussed, a technique that is useful in determining the sub band gap states in organic polymer solar cells. Capacitive photocurrent has been done to bulk heterojunction blend and compared with optical absorbance to observe the effect of sub band gap states that are not clearly significant in existing measurement techniques. Degradation of organic polymer solar cells is also discussed with the measurements done for range of thicknesses. 


\subsection{A brief introduction to history of organic solar cells}

The conventional solar cells were first developed in 1950s and been commercialized in 1960s and 70s[2]. These have many advantages as a clean, renewable energy source while increasing the efficiency and reducing the cost associated with it. As shown in the Figure 1.0, the photovoltaic technology has been evolved to a greater extent in efficiencies since mid 1970s.

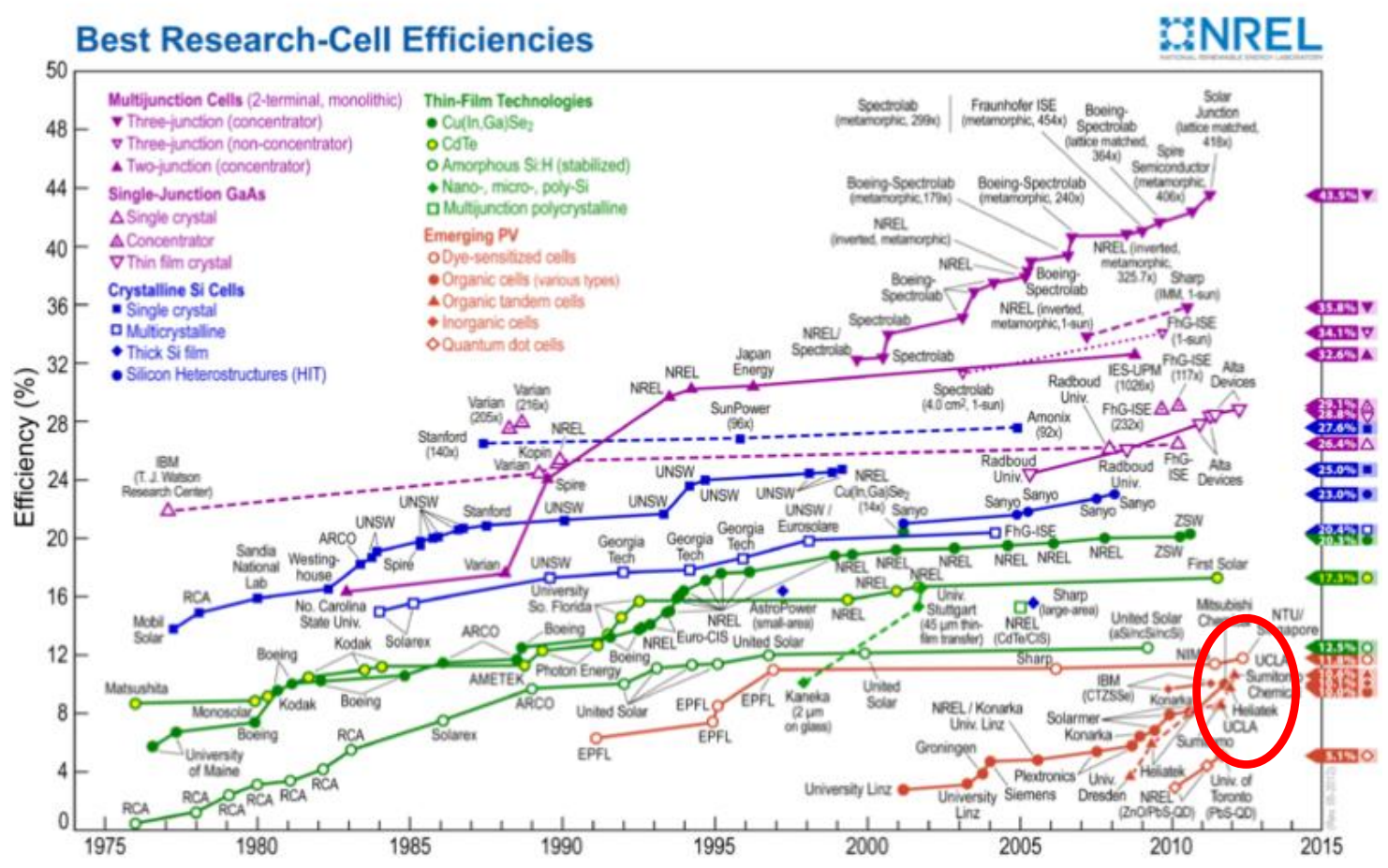

Figure 1.0: Evolvement of the Efficiency of the solar cells from 1975 to 2010.

Organic solar cells consist of semiconducting organic molecules. They are most famous among other materials because of easy fabrication and low cost manufacturing capabilities. This dissertation is focused on the development of organic solar cells denoted by the red circle at the right bottom of Figure 1.0. Even though the current efficiencies of organic solar cells are low, its potential to obtain higher efficiencies by tailor the energy levels with new organic materials make this a highly demanding area 
among researchers. The first successful organic based polymer solar cell has been reported by C.W.Tang in 1986 at the Kodak research laboratories in New York. In this work, a thin film photovoltaic cell using two-layer organic materials fabricated from copper phthalocyanine and a perylene tetracarboxylic derivative. A power conversion efficiency of about $1 \%$ has been achieved by Tang[3]. A breakthrough design by smart device physics came in 1995 using the organic bulk heterojunction structure. This simple method of mixing one type of polymer with another type simply enhances the interface which creates more e-h pairs (EHPs)[1]. Then another major breakthrough occurred in 2007 by the introduction of tandem structure concept that absorbs a broader range of solar spectrum[4] using a multi-layer device. Since then a positive trend has been shown in increasing efficiencies that currently claims $10 \%$ efficiency [5].

\subsection{General properties of organic semiconductors}

Organic semiconductors are the main backbone of the development of the organic solar cells. The applications of organic semiconductors depend on the molecular structure which has important electronic properties. Previously, these semiconductors have been extensively used in organic electronics such as organic field effect transistors (OFETs)[6]. Organic semiconductors can be divided into two classes according to their molecular structure: the low molecular weight materials and high molecular ones. High molecular weights are the polymers: the raw material of organic polymer solar cells. Both have a $\pi$ electron system formed by $\mathrm{p}_{\mathrm{z}}$-orbitals of $\mathrm{sp}^{2}$-hybridized $\mathrm{C}$-atoms in the molecules. Figure 1.1 shows the $\sigma$ and $\pi$ bonds of the simplest conjugated organic system: ethylene. The schematic diagram on the right shows the corresponding energy levels of $\pi$-conjugated molecule. The $\sigma$-bonds generate strong covalent chemical bonds 
by electron sharing from two carbon atoms. But $\pi$-bonds formed by $\mathrm{sp}^{2}$ orbitals create weak interactions. Due to this weak bonding strength, the lowest electronic excitations are the $\pi-\pi^{*}$ transitions (Figure 1.1) with an energy gap typically between 1.5 and $3 \mathrm{eV}$.

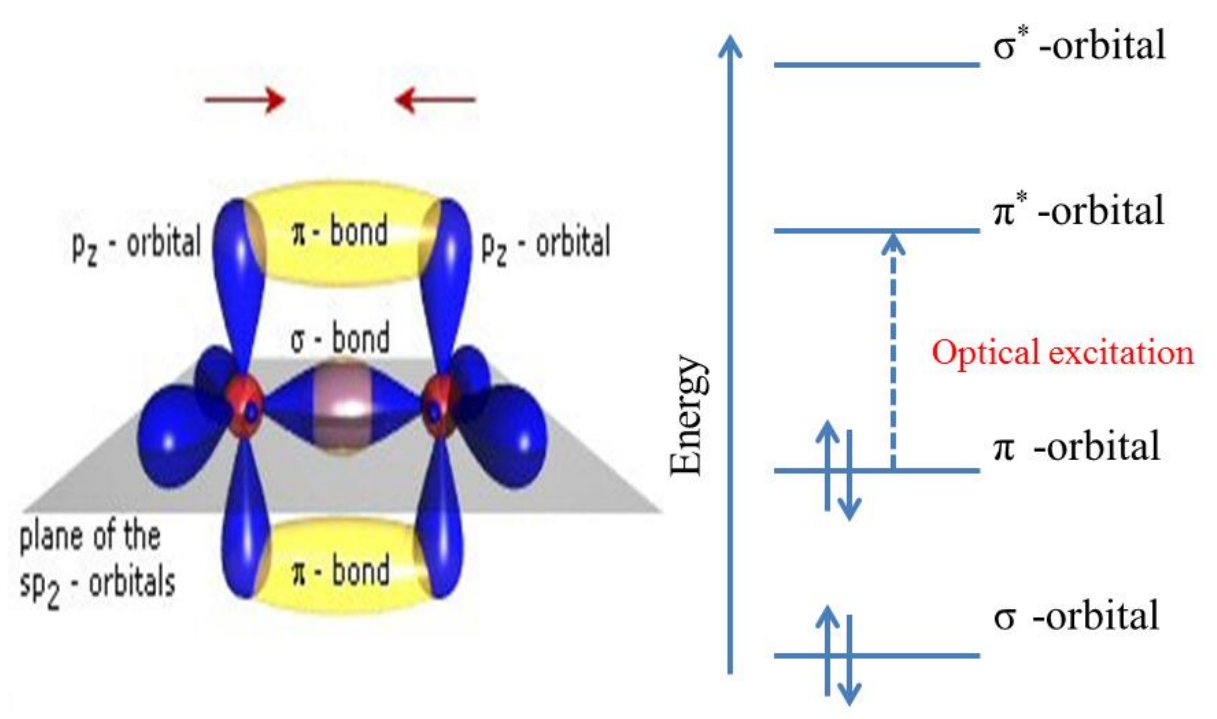

Figure 1.1: $\pi$-bond formation in $\mathrm{sp}^{2}$-hybridized carbon atoms in simplest conjugation system: ethylene $\left(\mathrm{CH}_{2}=\mathrm{CH}_{2}\right) \cdot \pi-\pi{ }^{*}$ optical transition shows the lowest energy excitation result in these conjugated polymers.

The $\pi$-bond orbital of highest occupied electrons called as HOMO and the $\pi^{*}$ orbital of lowest unoccupied electrons called as LUMO. This is similar to valence-conduction bands in inorganic semiconductors. This energy transition corresponds to the light absorption or emission in the visible region of the solar spectrum. The synthesizing techniques of these molecules lead to the degree of conjugation by which the energy gap can be controlled. The molecular structures (monomers) of the main organic 
semiconducting materials used in this work are shown in Figure 1.2. Poly-(3hexylthiophene) also commonly shortened as P3HT has an optical band gap of 1.9 eV[7].

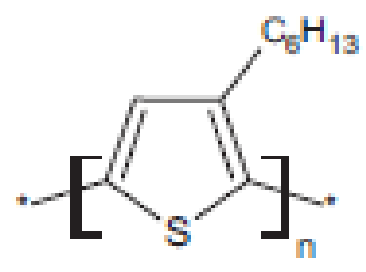

P3HT

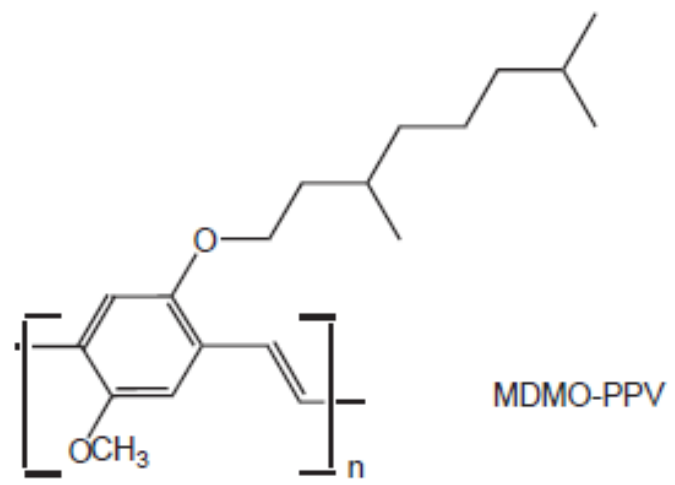

Figure 1.2: Monomer structures of the conjugated polymers used in this work.

In addition to P3HT, Poly[2-methoxy-5-(3,7-dimethyloctyloxy)-1,4phenylenevinylene] (MDMO-PPV or $\mathrm{OC}_{1} \mathrm{C}_{10}-\mathrm{PPV}$ ) is also used as displayed in figure 1.2. This conjugated polymer has an optical band gap at around $2.5 \mathrm{eV}$. In addition to these conjugated polymers, Poly[2-methoxy-5-(2-ethylhexyloxy)-1,4-phenylenevinylene] (MEH-PPV), poly(9,9'-dioctylfluorene-co-bis $\left(N, N^{\prime}\right.$-(4,butylphenyl))bis( $N, N^{\prime}$-phenyl-1,4phenylene)diamine) (PFB) [8, 9], Poly[2,6-(4,4-bis-(2-ethylhexyl)-4H-cyclopenta[2,1b;3,4-b0]-dithiophene)-alt-4,7-(2,1,3-benzothiadiazole)] (PCPDTBT)[10] have also been used as donor conjugated polymers. Since P3HT and MDMO-PPV are used in this work, they will be discussed further. The main optical difference between two conjugated polymers of $\mathrm{P} 3 \mathrm{HT}$ and MDMO-PPV is that the P3HT has a higher absorption profile compared to that of MDMO-PPV, even though both absorb in the visible region of solar spectrum. Due to this feature, P3HT is a better candidate in making efficient OPVs. The high absorption profiles associated with these materials $\left(\sim 10^{5} \mathrm{~cm}^{-1}\right.$ for P3HT and $10^{3} \mathrm{~cm}^{-1}$ for MDMO-PPV)[11] allow the option to make thinner devices for light harvesting. In 
contrast, the inorganics (mainly Silicon) have less absorption profiles $\left(10^{2}-10^{-5} \mathrm{~cm}^{-1}\right)[12]$ which limits from fabricating thin devices. The common feature both of these structures (P3HT and MDMO-PPV) have is the benzene. The benzene structure consists of the $\pi$ bonds that create weak delocalized electronic states in the semiconductors. Because of this weak delocalized electronic property, the organic semiconductors clearly distinguish from the inorganic semiconductors $(\mathrm{Si})$. This creates localized optical excited state or famously known "exciton" with a very high binding energy of $0.5 \mathrm{eV}$. In inorganic semiconductors, the light absorption creates free electron and hole to be collected by the electrodes. Excitons in organic materials created by light absorption are electrically neutral and have a high possibility to recombine before they dissociate. Due to this high binding energy of exciton, built-in electric field created by the difference of the metal work functions will not be affected for exciton dissociation. This is a major limitation of electricity generation using these types of photovoltaics in which the smart band-gap engineering must come into play.

\subsection{Photogeneration mechanism in organic polymer solar cells}

One of the main properties associated with organic semiconductors is the high absorption profile in the visible region as described in the above section. The light absorption results in a free electron and hole in the bulk region of the inorganic solar cell. Due to the creation of exciton, the charge transfer mechanisms in organic and conventional-inorganic solar cells have distinct differences, which will be discussed in this section. Organic solar cells are mainly categorized into organic semiconducting polymer solar cells, dye-sensitized solar cells and quantum dot solar cells based on different materials and structures they used in their development. In all these cases, the 
charge transfer mechanism, creation of excitons, dissociation of these excitons and finally the transport of free charge carriers are different compared to conventional solar cells.

Organic photovoltaics have major differences compared to conventional (inorganic) solar cells. In p-n junction inorganic solar cells, upon light absorption, free carriers are generated that diffuse to the outer electrodes that fits with general semiconductor theory. The creation of high coulomb potential exciton in inorganic semiconductor is less probable because of strong dielectric constant of the material. On the other hand, coulomb potential energy of an exciton in organic semiconductor lies in the range of tens of $\mathrm{K}_{\mathrm{B}} \mathrm{T}(\sim 500 \mathrm{meV})[13]$. High attractive energy of excitons in organic materials is because of the low dielectric constants ( $\sim 4)$ compared to that of inorganic materials (for Silicon 12). These low dielectric constant excitons in organics are also called as Frenkel excitons. The mobility of the charge carriers of these conjugated polymers is low $\left(\sim 10^{-3}-10^{-7} \mathrm{~cm}^{-1}\right)$ due to low crystallization structures. The low mobilities of these excitons led to low diffusion constants by Einstein relationship. Because of the low diffusion constant, the diffusion length $(=\sqrt{D \tau})$ lies around $10 \mathrm{~nm}[11]$ which is very low compared to that of inorganic solar cells[14] where D is the diffusion constant and $\tau$ is the diffusion lifetime. The diffusion length is the distance that excitons travel before they recombine. Due to the low diffusion length of the excitonic carriers, most will be radiatively recombined or trapped by sub-band gap states before reaching out to electrodes leading to low carrier extraction efficiencies. To complete photovoltaic conversion by collecting electrons and holes, there should be an asymmetry occurring within the material. This is achieved by introducing a material that has a higher electron affinity than the organic semiconductor. The most famous material used in 
almost every organic polymer solar cell is the derivative of methanofullerene or C60:PCBM. The band alignment of these two will be discussed in the next section of this work. Due to higher electron affinity of the fullerene, there is a high possibility that the electrons donated (created by light absorption) by the conjugated polymer is accepted by this material making an ideal candidate for n-type acceptor (accepts electrons) for OPVs. The basic structure of fullerene derivative used in organic solar cells is shown in the Figure 1.3.

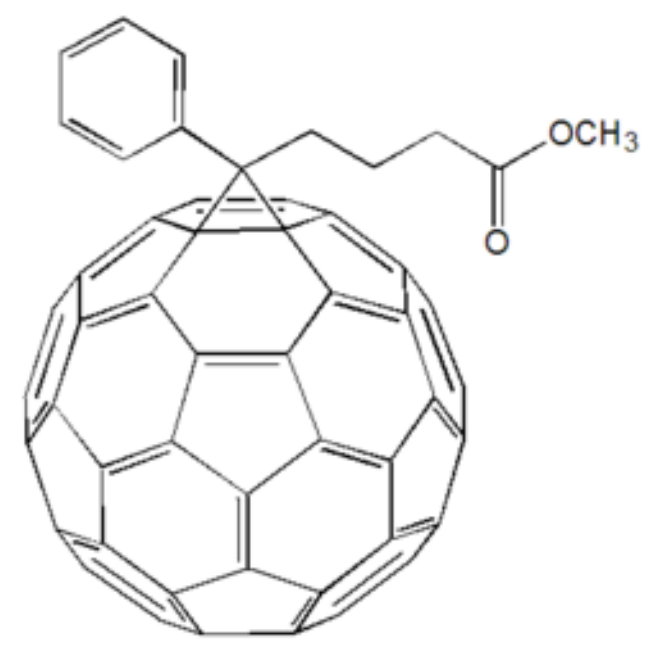

Figure 1.3: Monomer structure of the C60: PCBM fullerene derivative used in organic solar cell.

The fullerene is a derivative of C60 structure, which is created when a carbon atom is placed in every vertex of a truncated icosahedron. This is a polygon with 60 vertices similar to a soccer ball[ $[15,16]$ (this is famously called as buckyball or buckminister fullerene). This structure was first found by Richard Smalley group at Rice University in 1985[15]. However, this highly stable structure is unfortunately insoluble. To make it soluble, alkyl group is added to the buckyball which is used in almost every organic polymer solar cell. A detailed synthesis and functionalization of making the [6,6]-phenyl- 
$\mathrm{C}_{61}$-butyric acid methyl ester (C60:PCBM) is described by Hummelen et al[17]. In addition to C60:PCBM, poly(9,9'-dioctylfluorene-cobenzothiadiazole) (F8BT)[9], $(6,6)$ phenyl-C71-butyric acid methyl ester $\left(\mathrm{PC}_{70} \mathrm{BM}\right)[18,19]$ are the other popular candidates for electron accepting materials.

The excitons that are created around the distance of $10 \mathrm{~nm}$ or less will dissociate once they reach the interface of donor-acceptor in organic solar cell. A pictorial representation of this motion is shown in the Figure 1.4 along with the band alignment.
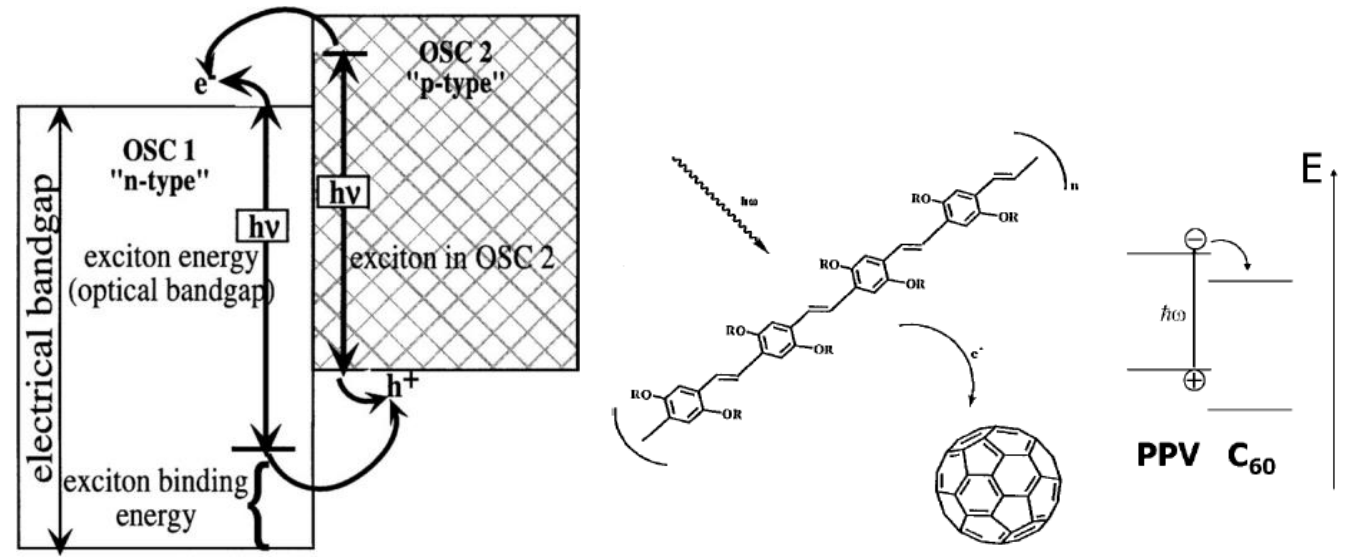

Figure 1.4: Band alignment of polymer (p type) and PCBM (n type) showing the charge transfer at the p-n junction interface. OSC 1 and OSC 2 are organic solar cell of n-type material and p-type material respectively.

The electron donating material is the conjugated polymer. And fullerene derivative (C60:PCBM) is the electron acceptor. This dissociation may occur at the trap sites in bulk of the material but it is not the driving mechanism to obtain such an efficient photoconversion. However this dissociation must not be neglected in these organic molecular structures with weak localized forces. The major dissociation occurs at the interface between the donor and acceptor where the binding energy of the exciton is 
lowered by the band offset of donor and acceptor[14, 20]. This separation leads to an electron in the acceptor and a hole in the donor material still bound by a weaker binding energy which is called charge transfer exciton (CTE)[21, 22]. This is also called the geminate e-h pair[23]. The distance between this bound electron and hole is several nanometers that give a very high electric field $(\sim \mathrm{MV} / \mathrm{cm})$. Then they dissociate into free carriers leaving a hole in conjugated polymer and an electron in fullerene. When the time scales are considered, the charge separation process occurs within sub nanosecond ( ns) time scale to form free electrons and holes. This includes forming the CTE state which is in sub picoseconds( $\sim \mathrm{ps})[24]$. Next stage is the transport of these free carriers via polymer and fullerene phase by the built-in electric field and concentration gradient due to absorption profile. On the way to the respective electrodes, these charge carriers can be recombined again with another free electron and hole created from a different molecule. It is also reported that this charge transport through the fullerene is occurred by so called a hopping mechanism[25]. At thermal equilibrium, free holes tend to move towards the metal with low work function (cathode) and electrons move towards the high work function metal (anode). The band alignment of the organic polymer solar cells used in this work is shown in Figure 1.5. 


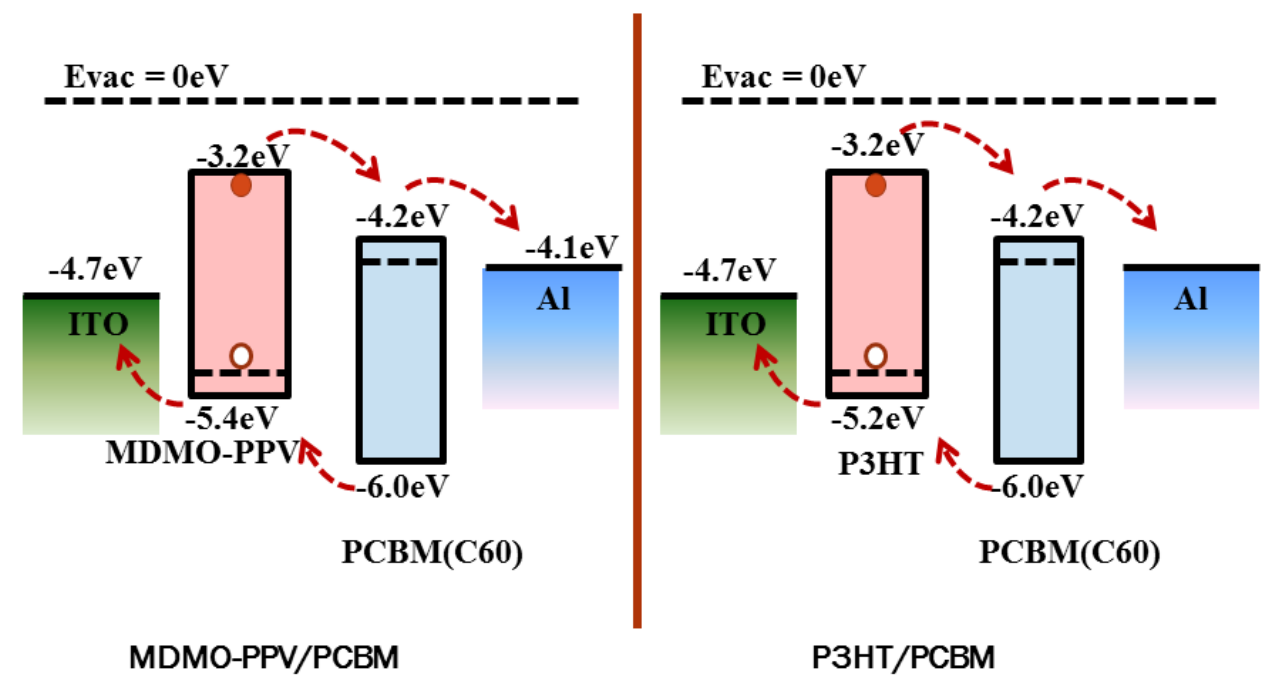

Figure 1.5: Band alignment of MDMO-PPV: PCBM (left) and P3HT: PCBM (right)

systems showing the charge transfer and the energies with respect to vacuum $[7,26]$.

\subsection{Materials used in fabricating organic solar cells}

Other than the materials used as the active layers, choice of metal electrodes are also important in having an efficient metal-semiconductor junction. The first aspect is, at least one of the contact metal electrodes must be optically transparent, at least in the region where most light is absorbed by the semiconducting polymers. Indium tin oxide (ITO), fluorine doped tin oxide (FTO) and doped zinc oxide are transparent conducting films that are widely used in device fabrication. $100 \%$ transmission in visible region, high electrical conductivity and having comparatively high melting points are some of the attractive properties of these materials that can be used in polymer solar cells. The other 
collecting electrode is aluminum (Al) which is commonly used in many semiconducting devices for an efficient charge extraction. Gold $(\mathrm{Au})$ is also used as the anode since it is a better candidate in having a non-oxide metal electrode compared to aluminum. The use of ITO is highly desirable because the HOMO level of electron donor is closer to the work function of ITO which will make the hole transfer ohmic. Similarly, the Al work function is closer to LUMO of electron acceptor so the electron transfer will be ohmic from PCBM to Al. In addition to two metal electrodes in organic solar cells, thin buffering layers are added in between metal/semiconductor interface to have smooth charge extraction and to avoid opposite charge leaking through the interface. Material to be inserted as hole or electron buffer layer is decided by selecting proper type of semiconductor. A thin layer of this material is deposited to make it fully optically transparent. P-type oxides such as 10nm of thin Nickel oxide (NiO)[27, 28], Vanadium oxide $\left(\mathrm{V}_{2} \mathrm{O}_{5}\right)$, Molybdenum oxide $\left(\mathrm{MoO}_{3}\right)$ [5, 29], Zinc oxide ( $\left.\mathrm{ZnO}\right)[30]$ and Titanium oxide $\left(\mathrm{TiO}_{\mathrm{x}}\right)$ [31] are commonly used for this purpose. Furthermore, a thin layer of n-type $\mathrm{C} 60: \mathrm{PCBM}$ is also used as an interfacial layer between the active layer and aluminum electrode [32] to enhance the electron extraction from the active device. However, the most promising buffer layer used in preparing organic solar cells is the PEDOT: PSS, poly (ethylene-dioxythiophene) doped with polystyrenesulfonic acid. This aqueous solution is deposited in between metal and active layer to improve better charge extraction from the active layer $[11,33,34]$ and improving the smoothness with less pin holes in ITO[35] . Transparency in visible region is achieved by depositing a thin layer of this material. This has a high electrical conductivity as well as a good thermal stability that acts as a hole transporting layer. Main disadvantage of using this material in organic 
solar cells is the degradable addition of water into the device. An ultra-thin layer of Lithium fluoride $(\mathrm{LiF})$ is used in the back side of the cell between the aluminum and the active layer to have a better charge transfer from the active area by reducing the work function of the metal[34, 36]. A very thin layer $(<1 \mathrm{~nm})$ is deposited in order to avoid insulating the barrier of this material. However, the exact role of this thin layer is not known.

\subsection{Recombination losses in organic solar cells}

Recombination is the main factor that prevents the electron and holes reaching the electrodes. The recombination mechanism of the organic solar cells mainly occurs at the interface where they dissociate, while in inorganic solar cells this occurs at the bulk as well as at the p-n junction. Two main types of recombinations are possible in organic polymer solar cells. First type is the monomolecular recombination which is the recombination due to electron and hole that separate from the same exciton. This recombination can also occur by recombining charge carriers with trap sites within the bulk[37]. The other type is the bimolecular recombination. Bimolecular is recombining the electron or hole with another e-h other than the molecule they are originated from due to absorption. The first type is more common since the possibility of recombination at the interface is higher than the bulk of the material. Also the linear dependence of photocurrent to light intensity shows that the bimolecular recombination is not the dominant factor in recombination mechanism[38]. Also Street et al. (2010) described the bimolecular recombination is the recombination of electron and hole in either polymer or fullerene by each other before they diffuse to the donor-acceptor (D-A) interface. This recombination therefore depends on the optical generation rate[23]. The bimolecular 
recombination is also described as the Langevin recombination[39]. Another possibility is the Geminate charge transfer excitons. Once a photogenerated exciton created and diffused to D-A interface they dissociate and create a bounded electron in PCBM and a hole in polymer which is called the geminate e-h pair or commonly charge transfer excitons (CTE). Then the built-in electric field, occurred within the device, lower the barrier (binding energy, BE) between this geminate pair which ultimately create a free electron and a hole. There is a possibility that these free carriers meet at the D-A interface and recombine with an electron and hole creating a CTE. Another possible recombination is that when the carriers generate more close to metal interface in thin devices, there is a possibility that they can diffuse against the built-in electric field and recombine at the metal-semiconductor interface states.

\subsection{Mobility of the charge carriers in the organic solar cells}

Mobility of the charge carriers is another crucial factor in charge collection efficiency of the organic solar cells. In order to obtain an efficient charge extraction from the electrodes of a solar cell, it is important to analyze how the charge carriers transport within the bulk of the devices. A more practical and experimental technique would be to find the carrier mobilities of these structures. Standard techniques used to find the carrier mobilities of amorphous polymers and organic structures are Time of Flight (TOF), Dark injection (DI) transient and Current- voltage (J-V) measurements. These existing methods developed to find the carrier mobilities are associated with a semiconducting material that has no traps with a flow of space charge limited current (Trap-free SCLC). In other words, it is assumed that the trap states created by the metal-semiconductor interfaces are

negligible and the injected electrode (ITO, the optically transparent electrode considered 
as the injecting electrode in these measurements) has no significant resistance. Thus, any applied external bias to the device falls across the active device area giving the electrodes pure ohmic[40, 41]. The following section of this dissertation briefly describes these techniques.

\subsubsection{Dark Injection (DI) Technique}

The dark injection transient current technique (DI) [42-44] is often used to measure the charge mobility of organic solar cell structures. This method is based on applying a voltage pulse to the sample in a dark environment and recording the current flow through the device. This is displayed as a voltage across a load resistor on an oscilloscope. Mobility can be calculated from this transient time by measuring how long it takes for these charge carriers to drift across the sample. One disadvantage of this technique is that the DI current technique involves unipolar devices. That is only majority carriers dominate in current flow through the device. Therefore using this technique, only one carrier type can be investigated. Dark injection current with photo excitation and dark injection current with electric excitation are common methods to investigate carrier mobilities.

\subsubsection{Space charge limited current-voltage measurement}

This technique assumes that the injecting contact is ohmic, and steady state current is limited by the space-charge in the sample which is proportional to the mobilities of the carriers. Steady state current density $\mathrm{J}$ as a function of applied voltage is given by[43, 45] 


$$
J(V)=\left(\frac{9}{8}\right) \varepsilon \varepsilon_{0} \mu \frac{V^{2}}{d^{3}}
$$

Where, $\varepsilon$ is the dielectric constant of the material, $\mathrm{V}$ is the applied voltage and $\mathrm{d}$ is the device thickness. I-V characteristics obtained for this method is the standard technique of measurement which is described in detail in the next chapter. The transient currents generated due to applied bias (V) stay constant while the carriers drift across the device (nondispersive). In this case, the charge transport mobility is field dependent and can be used in organic materials. If the voltage values and the corresponding currents are extracted from the J-V curve in Equation 1.1, one would easily obtain the mobility of the material $\mu$. This relation is also called as the Mott-Gurney law.

\subsubsection{Time of Flight Technique (TOF)}

Another effective method to investigate the charge transport mobilities of organic structures is the time of flight (TOF) technique[46]. The main advantage of this method is the ability to measure both electron and hole mobilities. It has become famous after applying to the organic crystals by Kepler et al [47] and LeBlanc et al [48] in 1960s. In this method, a thin sheet of excited states (electron-hole pairs) is generated near one of the non-injecting contacts, usually the transparent contact by absorption of a short duration strong optical laser pulse[49]. If it is an asymmetric device, a reverse bias is applied to the device, by using a small work function electrode as the anode and a large work function electrode as the cathode. If the laser is incident on the anode, then, under the influence of the applied electric field, electrons combine at the anode and a sheet of remaining holes charge carriers will move across the device under the electric field, eventually reaching the counter electrode. The holes mobility can be detected in this way. 
On the other hand, if the laser is shone on the cathode, the hole combines with this electrode and leaves the electrons to move under the applied field and drift to the anode electrode, hence the electron mobility can be obtained. The experimental setup used to measure the carrier mobility is shown in the Figure 1.6.

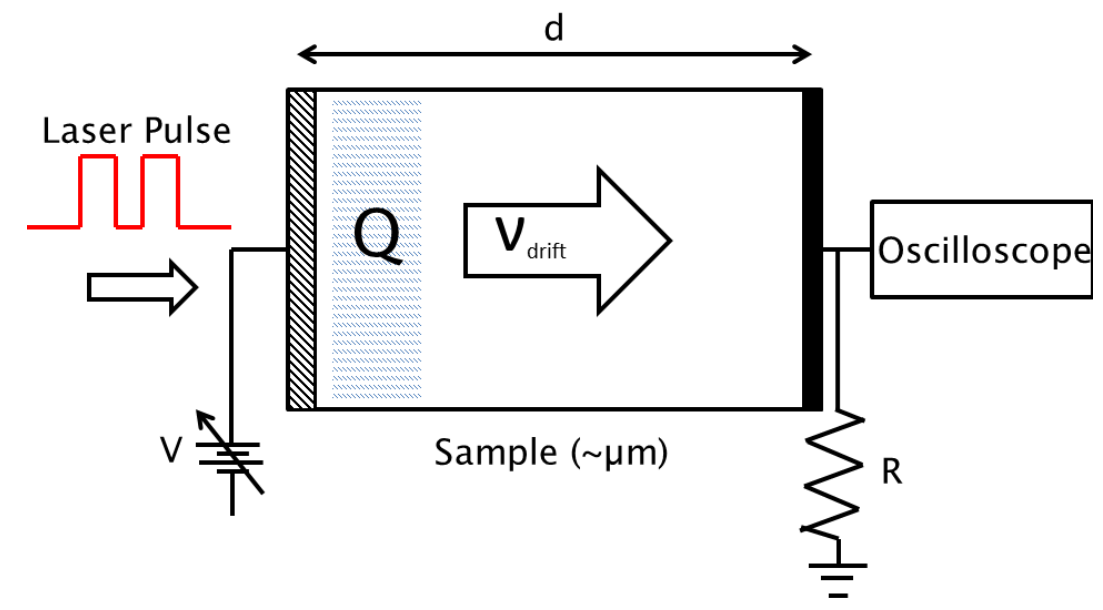

Figure 1.6: Experimental setup used in time of flight (TOF) measurement.

A sheet of charge carriers is drifting across the sample under the electric field (shown as blue column in Figure 1.6). In an ideal situation, a constant photo generated current passes through the device which suddenly falls to zero as the sheet of charge carriers reaches the counter electrode. 

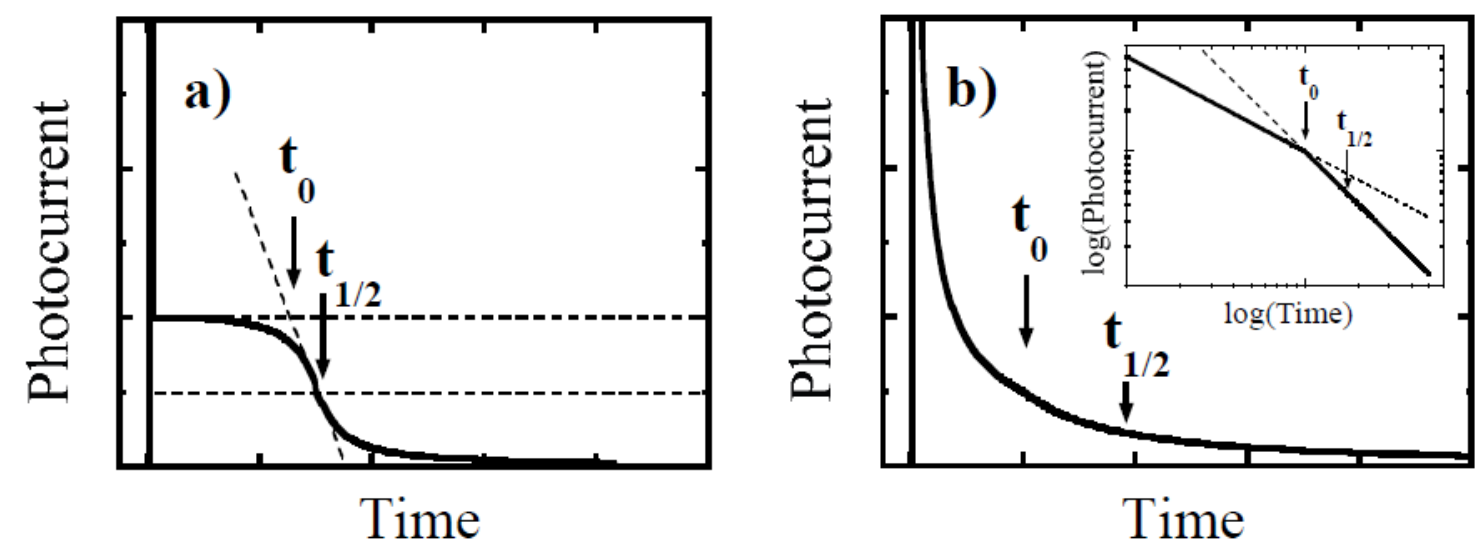

Figure 1.7: Transient currents of (a) nondispersive and (b) dispersive obtained using TOF measurements. Inset of (b): double log rhythmic plot[50, 51].

In a real situation, however, ToF transients would not be in this ideal shape (Figure 1.7 (b)). In real time measurement, this thin sheet of carriers has a finite depth into the bulk due to the absorption depth of the photons. Because of significant diffusion component of the carriers and the variation of the carrier mobility occurred within the material leads to dispersive transient curve shown in Figure 1.7(b). The mobility of the sample is related to the transit time which can be found in above transient curves. The relationship used in determining the mobility is given by the equation below.

$$
\mu=\frac{d^{2}}{V \cdot t_{t r}}
$$

Where $\mathrm{d}$ is the active device thickness, $\mathrm{V}$ is the applied voltage and $\mathrm{t}_{\mathrm{tr}}$ is the transit time. More detailed discussion of the measurement setup will be discussed in next chapter.

\subsection{Device Configuration of organic solar cells}

The device configuration of a typical organic solar cell should be optimized in order to get the highest possible charge collection efficiency. Therefore, the device 
physics and band-gap engineering are important factors constructing a device configuration.
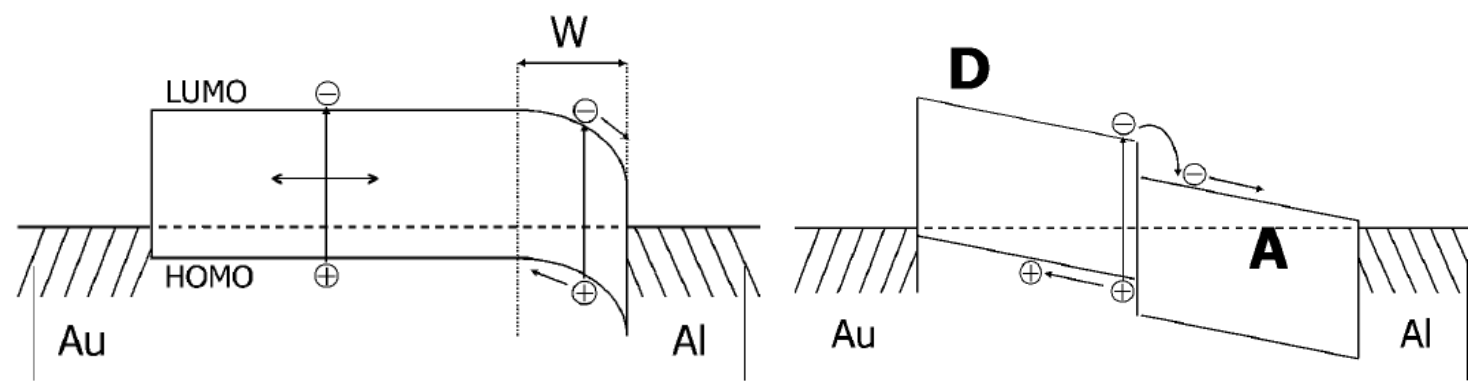

Figure 1.8: Device configuration of single layer (left) and bilayer (right) devices showing the charge transfer at the p-n junction interface and at the bulk.

Structures at the beginning of this field were fabricated as a single layer metal/semiconductor/metal (MSM) configuration[52]. The excitons created are diffused to the semiconductor metal interface and dissociate at the interface. Since the diffusion length is very small, only a small fraction of excitons make it to the interface. The energy for the separation of the excitons is obtained by the band offset at the metal/semiconductor interface and therefore only those excitons are contributed for the photocurrent generation. This configuration is also called as planar configuration in which the conjugated semiconducting polymer is deposited on top of metal that has lower work function. Then a higher work function metal (Al) is deposited on top of the semiconductor. The bilayer configuration comes with the donor-acceptor (D-A) concept. This has improved the device performance by improving charge extraction. The main advantage of this configuration is the efficient charge separation due to band offset of donor and acceptor material. The binding energy between the exciton is lowered by the band offset of donor and acceptor materials, but still, the short exciton diffusion lengths 
limit carrier generation of this configuration. Only the excitons much closer to the D-A interface dissociates contributing to the current. Two materials are stacked as separate single layers on top of each other between two metals that have different work functions.
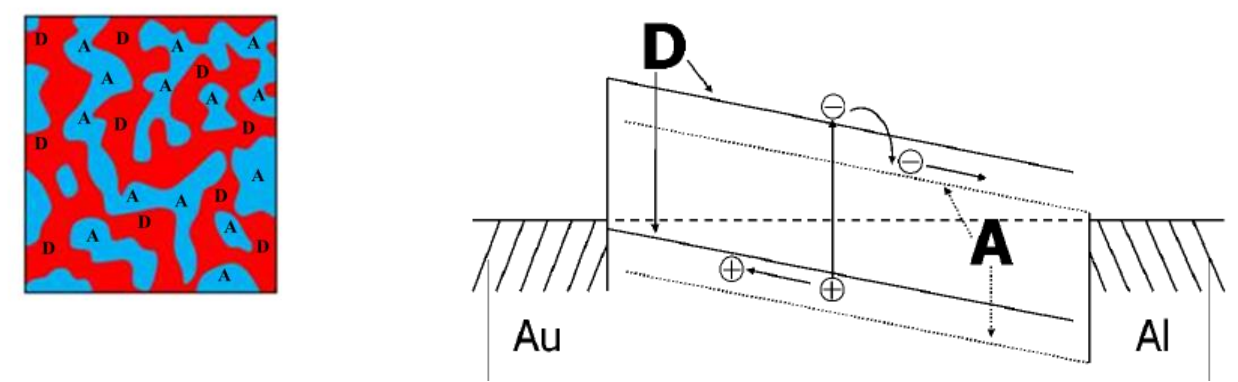

Figure 1.9: Device configuration of bulk hetero junction-BHJ (left) and bilayer (right) devices showing the charge transfer at the p-n junction.

To overcome the issue of diffusion limit, the bulk heterojunction (BHJ) concept is used. This is achieved by simply mixing the donor material with the acceptor material as a blend that enhances the D-A interface area. In this way, the diffusion lengths of the excitons reduces tremendously[1, 53, 54]. This breakthrough improvement of the device architecture is still used in almost every polymer solar cell device.

\subsection{Organic solar cell device fabrication}

Fabrication of an organic solar cell does not involve sophisticated and expensive methods. However advanced methods can be used to improve the performance and the stability of the device. For example, fabrication of organic solar cell does not necessarily need an inert gas $\left(\mathrm{N}_{2}\right)$ filled glove box, but making a device inside a glove box under inert atmosphere produces a more stable device with less degradation. The only advance technique needed in this process is the deposition of the top metal electrode. Usually, 
thermal or electron beam evaporation is used for the deposition of the top contact that associates high vacuum deposition. All the devices used in this work are fabricated with electron beam (e-beam) evaporated metal contacts. Thickness of about $60 \mathrm{~nm}$ is deposited under a vacuum of $10^{-7}$ Torr. Source material is melted by an electron beam and evaporated on the substrate which is located above the crucible liner. The electron beam is directed towards the deposition source by an electric field. The deposition rate and the thickness are measured by a crystal sensor located above the emitter assembly. A long mean free path $(\sim 60 \mathrm{~m})$ of evaporated source molecules is achieved having a high vacuum. The crucible used in these depositions is graphite. Materials deposited using this technique is Aluminum, Gold and Lithium fluoride. Lithium fluoride and aluminum are deposited by moving crucibles with evaporation sources without breaking the vacuum.

The starting material is the bottom metal electrode indium tin oxide (ITO) which has a sheet resistance of about20-25 $\Omega / \square$ purchased form Sigma Aldrich Inc. Cleaning of the indium tin oxide substrate using acetone, isopropanol (IPA) and deionized water (DI) for 30 minutes each is the first step. This is necessary in order to remove any residue/contaminants and to improve the adhesion of the top layers onto ITO. Then the substrate is annealed for about 30 minutes at $100^{\circ} \mathrm{C}$ in air to remove the residues of solvents. Next, the ITO substrate is patterned by immersed in concentrated solution of Hydrochloric acid $(38 \% \mathrm{HCl})$. Zinc catalyst can also be used which accelerates the etch rate. Without the catalyst, etch rate is about $8 \mathrm{~nm} / \mathrm{min}$ (with catalyst the rate increases by a factor of 2 that gives $16 \mathrm{~nm} / \mathrm{min}$ ). An ordinary tape is used to mask the ITO substrate to avoid the electrical shortings when making the electrical contacts. Once ITO is etched, the patterned substrate is washed off using DI water and covered by a petri dish until it is 
fully dried. Then a thin layer of PEDOT-PSS (poly(3,4-ethylenedioxythiophene)poly(styrenesulfonate)) layer is deposited using the spin coating technique. Substrate is rotated in a high speed vacuum chuck while the solvent is deposited from the top. The centrifugal force is applied to the solvent that spreads and spins to achieve a uniform thickness throughout the substrate. Chemat Technology KW4 dual stage coater is used in fabricating the solar cells. This technique is the cheapest and widely used wet processing method in depositing layers in organic solar cells. The time duration and the spin speed are varied according to the recipe. PEDOT-PSS layer is deposited at a spin speed of 5000 $\mathrm{rpm}$ for $\sim 1$ minute (or less) to achieve a thickness of about $30 \mathrm{~nm}$. As soon as thin layer is dried, it is annealed at $140^{\circ} \mathrm{C}$ in an oven for about 10 minutes. After cooled down to room temperature, the substrate is transferred to nitrogen filled glove box to deposit the blends of MDMO-PPV: PCBM and P3HT: PCBM. The PEDOT-PSS is deposited in atmosphere due to the fact that it reacts with inert gas. Different spin speeds are used to achieve different thicknesses for the deposition of active blend layer on top of PEDOT-PSS for about 1 minute, covered by a petri dish until fully dried. Next the device is annealed for $80-100^{\circ} \mathrm{C}$ inside the glove box for 30 minutes. Once it is cooled down to room temperature the sample is transferred to the evaporator for the top contact deposition. Again a shadow mask made out of aluminum foil is used to make windows of metal on top of active layer. Flow diagram of the fabrication is shown in Figure[55]. 

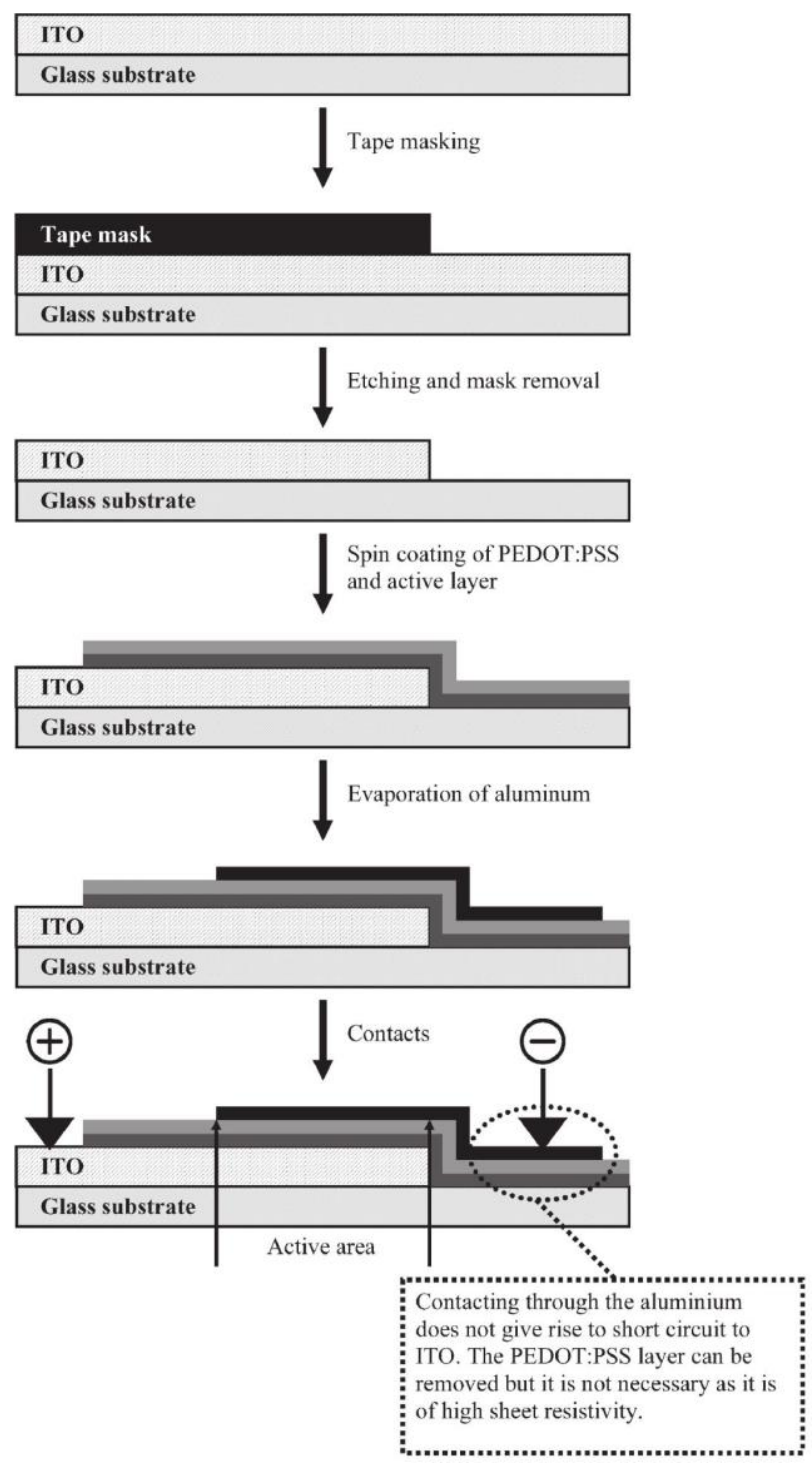

Figure 1.10: Flow of the fabrication of layers in organic bulk heterojunction solar cells.

As soon as the top contact is deposited, the sample is transferred to the cryostat. Electrical contacts are made by attaching thin copper $(\mathrm{Cu})$ wires with silver $(\mathrm{Ag})$ epoxy. Then the device chamber is purged with inert gas $\left(\mathrm{N}_{2}\right)$ or pumped for high vacuum for the measurements. A complete and detailed fabrication process is also described by Wan Ma et al.[56] The complete schematic of the device structure used in this work is shown in Figure 1.11. 


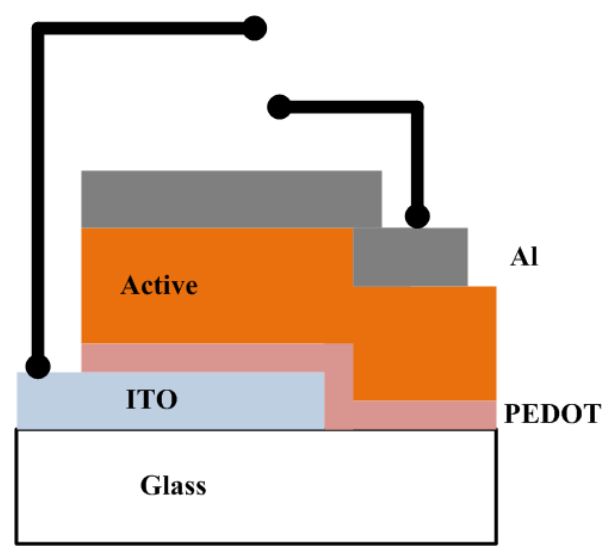

Figure 1.11 Basic device configuration of the organic solar cell. 


\section{CHAPTER II: INITIAL CHARACTERIZATION OF ORGANIC SOLAR CELLS}

A major part of this work was conducted with the photocurrent spectra that was obtained using a white light excitation source connected to a monochromator. Therefore, it is worth describing this custom made characterization system in this chapter. The tunable ultra-fast pulse laser system is another excitation source that is used in mobility measurements. This laser system pumps an optical parametric amplifier (OPA) 800CF, which will also be discussed in the first section. Next, the optical characterization of the P3HT: PCBM organic solar cells will be discussed along with the electrical characterization and the performance of the devices. Time-of-flight technique that measures the mobility of the organic solar cells will also be discussed in a later section of this chapter.

\subsection{Optical excitation sources used in characterization of the solar cells}

Photons flux spectra of light excitation sources that are available are not closely match with the solar photon flux due to difference of filament temperatures in incandescent sources. Spectral photon flux of the sun is described by blackbody radiation as shown in Figure 2.1. 


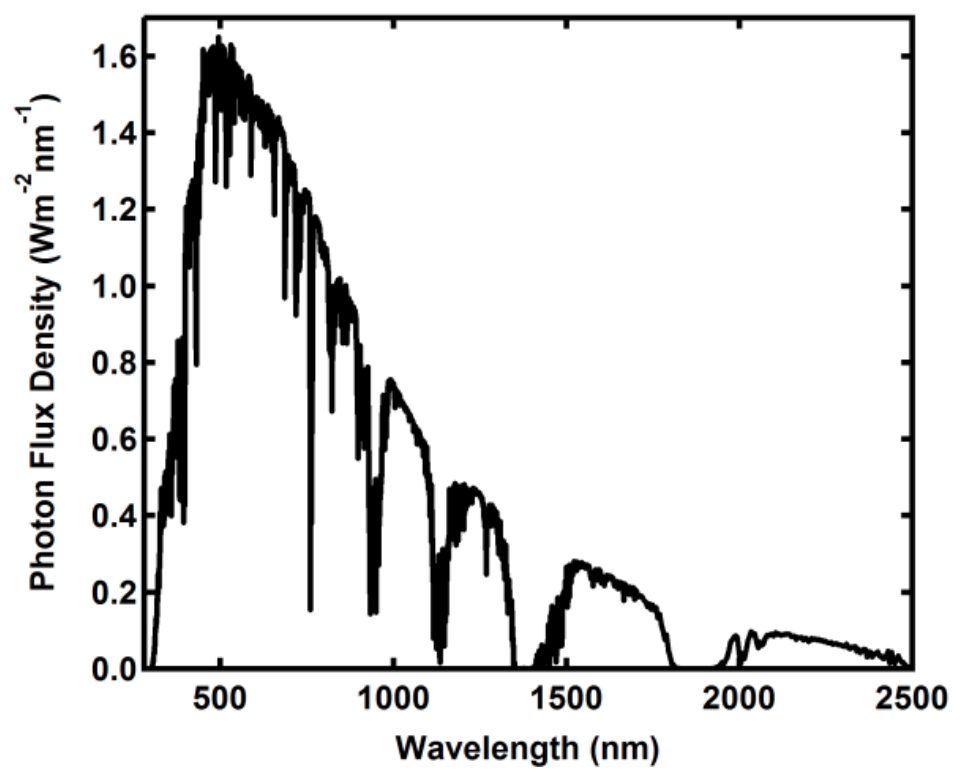

Figure 2.1: Spectral photon flux of the sun

The electrical characterization of solar cells was done using a solar simulator of TH400 lamp at the power of 100W. This matches with sun's solar spectrum of AM 1.5G which is about $100 \mathrm{~mW} / \mathrm{cm}^{2}$.

\subsubsection{QTH white light system}

The excitation source of the photocurrent setup is 300W quartz tungsten halogen (QTH) white light. The spectral irradiance of this light source is in the range of 400 to $800 \mathrm{~nm}$, at a distance of $50 \mathrm{~cm}$ (Figure 2.2). 


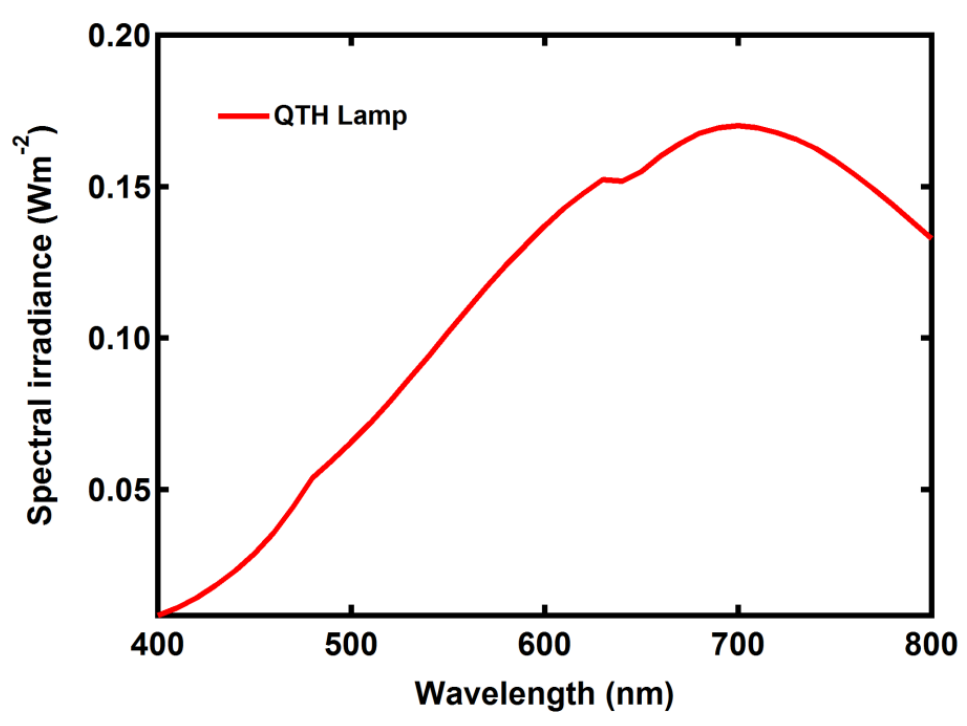

Figure 2.2: Spectral irradiance of the QTH white light source measured by Silicon photo detector.

The spectral irradiance gradually increased from 400 up to $700 \mathrm{~nm}$, and then droped off. According to the spectral irradiance curve, the maximum intensity of the output light from the source was given at $720 \mathrm{~nm}$. This light source was chosen because of minimum distortions of the spectral irradiance that occurred within the visible region of the spectrum.

\subsubsection{Spectra Physics ultra-fast pulse laser system}

The laser system is a solid state femtosecond pulse laser that outputs constant $800 \mathrm{~nm}$ with the power of $\sim 1 \mathrm{~W}$. This high power constant pulse beam is output to an optical parametric amplifier which has the wavelength tuning capabilities from 300 to $2400 \mathrm{~nm}$. This wavelength tuning is done by having a non-linear crystal (Beta Barium Borate (BBO)) in laser path that changes the wavelength by altering its orientation. The gain medium of this laser is Ti: sapphire (titanium-doped sapphire) crystal which sits in a 
cavity that has an acoustic optical modulator (AOM) to achieve phase matching. The result is a pulse train with a pulse width of 120fs. The repetition rate of the pulse is $1 \mathrm{kHz}$ which is fixed. A detailed description of this laser system is available in Shah (2012)[57]. The output of $800 \mathrm{~nm}$ at $4 \mathrm{~mW}$ power was illuminated at solar cell device for time of flight (TOF) measurements to investigate the carrier mobility of P3HT: PCBM devices.

\subsection{Photocurrent characterization system}

This system is basically divided into several parts. They are the light source (QTH: Quartz-Tungsten-Halogen white light), monochromator, focusing lens and the electronics. The schematic of the setup is shown in Figure 2.3.

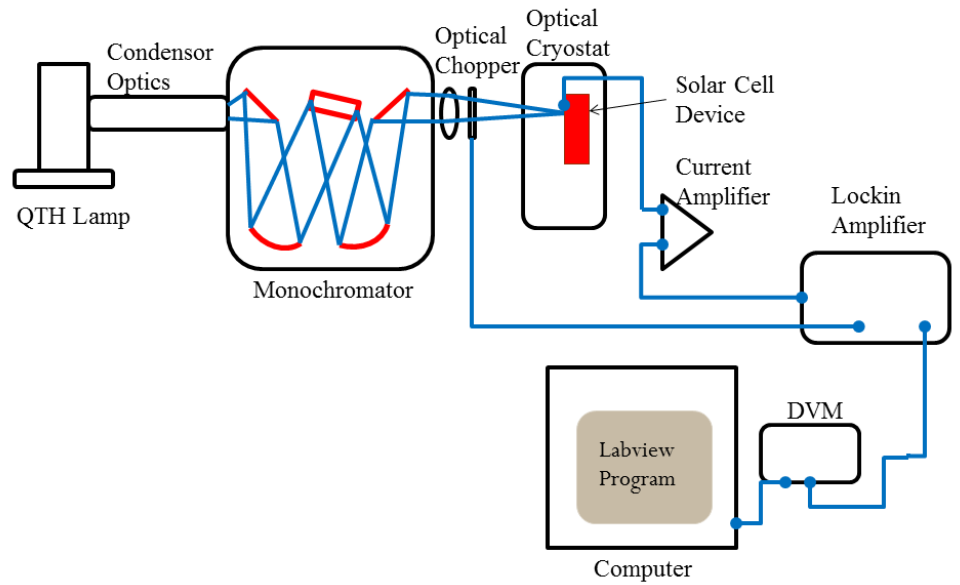

Figure 2.3: The Schematic setup used to measure the photocurrent spectra of organic solar cells.

The white light system is connected to the monochromator using condenser optics that matches the $\mathrm{f} / \#$ ( $f$ number). ' $f$ ' number describes the light gathering power of lens that is associated with any optical instrument. This is basically the ratio of the focal 
length to the diameter of the second reflective mirror in the monochromator (This can be the diameter of the lens or an input mirrors in the monochromator). For this system, this value is 6.50. Matching $f$ numbers result in high throughput of the power from the light source. A monochromator is introduced so that all the light from the condenser optics is obtained at the entrance mirror. Then white light is $100 \%$ reflected without losing any power to a grating of 1200 grooves per millimeter with a blaze wavelength of $750 \mathrm{~nm}$ that disperses the light into all the wavelengths. Then this light is collected by another mirror and reflected to the output slit. Output light is calibrated with a standard fiber optic spectrophotometer. Grating of the monochromator is controlled by the Labview program in a computer, which has the capability to change the scan rate. An optical chopper is introduced between the sample and the output slit of the monochromator that introduces different light pulses with frequencies that sink into a dual phase lockin amplifier for alternative photocurrent measurements. The electronics consists of a current amplifier, dual phase lockin amplifier and a digital voltmeter. All the electronics are connected to the PC to obtain the photocurrent spectra in direct (DC) and alternative (AC) modes that controls by a Labview program. This system also has the capability to produce incident photon to current conversion efficiency (IPCE or QE\%) spectra of a solar cell by taking the active area of the device. The standard solar spectrum is fed into the program by a data file to calculate QE. IPCE describes how many electrons are generated with respect to incident number of photons in a solar cell device[58] that is denoted as a percentage (\%). The IPCE is defined as

$$
I P C E=\frac{1240}{\lambda} \frac{I_{s c}}{P_{\text {in }}}
$$


Where $P_{i n}$ is the incident power from the light source $\left(\mathrm{Wm}^{-2}\right), I_{s c}$ is the short circuit current density $\left(\mu \mathrm{Acm}^{-2}\right)$ and $\lambda$ is the wavelength (nm) [58].

\subsection{Normalization procedure of the photocurrent}

The photocurrent obtained from the solar cell devices have to be normalized in order to observe the exact features of charge transfer states. Since the power spectrum of the light sources do not have constant wavelength dependence, this normalization procedure is necessary to see the correct features due to photocurrents.

First, number of photons was calculated for the wavelength range from 400 to $800 \mathrm{~nm}$. The energy of a photon was calculated by

$$
E=\frac{h c}{\lambda}
$$

Where $\mathrm{h}$ is the Planck constant given by $6.626 \times 10^{-34} \mathrm{~J} . \mathrm{s}$ and $\mathrm{c}$ is the velocity of light in vacuum given by $3.0 \times 10^{-8} \mathrm{~m} / \mathrm{s}$. Next, the total energy of photons was obtained by multiplying the energy of a photon by number $\mathrm{N}$, where $\mathrm{N}$ is the number of photons. Total energy of $\mathrm{N}$ photons equals to the power output $\mathrm{P}=\mathrm{P}(\lambda)$. Then dividing $\mathrm{P}(\lambda)$ by an energy of a photon will equal to $\mathrm{N}$ (number of photons).

$$
\frac{P(\lambda)}{E}=\frac{P(\lambda)}{h c / \lambda}=\frac{\text { No.of photons }(N)}{\text { sec.cm }}
$$

The number of photons emitted from 400 to $800 \mathrm{~nm}$ is shown in Figure 2.14. 


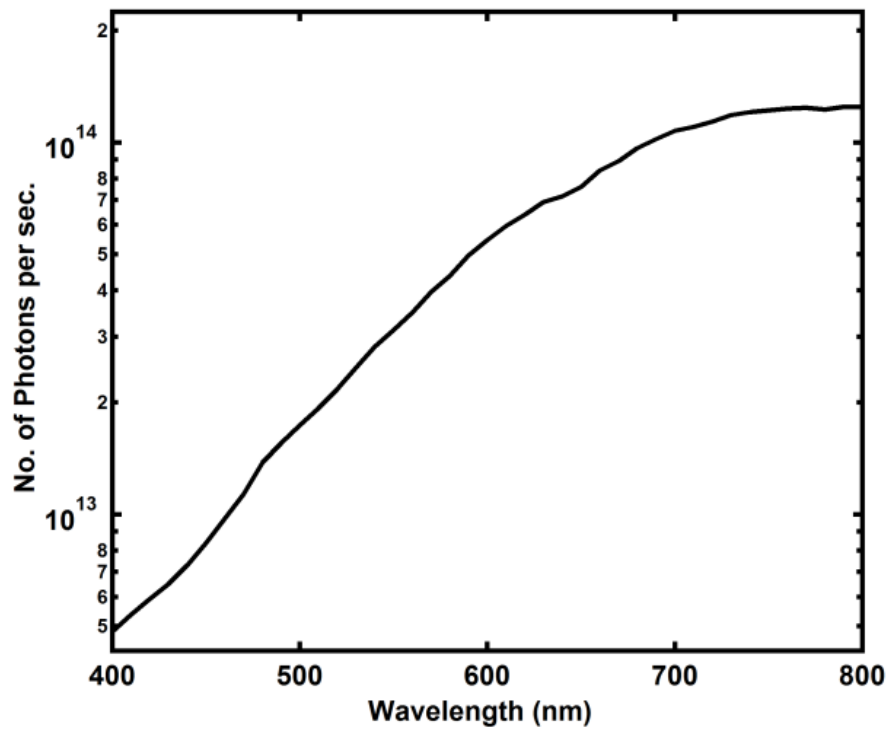

Figure 2.4: Number of photons calculated within the region of visible spectrum by the light absorption for the QTH light source.

Then, the number of incident photons was calculated by multiplying the number of photons with $[1-\exp (-\alpha d)]$, where $\alpha(\lambda)$ is the absorption coefficient and $\mathrm{d}$ is the active layer thickness. Finally, the photocurrent $\left(\right.$ Coulombs $\left./ \mathrm{cm}^{2} \mathrm{~s}\right)$ obtained from the device was divided by the number of incident photons to obtain the normalized photocurrent. This value equals to IPCE shown in Equation 2.1. The next chapter discusses the theoretical model used to investigate the physical mechanism of the data.

\subsection{Optical characterization of the organic solar cells}

\subsubsection{Atomic force microscopy measurements of organic solar cells}

Atomic force microscopy studies were carried out for the P3HT: PCBM blend materials with different thicknesses to study the morphology of the active layer. Following graph shows the AFM images of two different samples with the same composition (5:4) but with different thicknesses (left: $2 \mu \mathrm{m}$ and right: $100 \mathrm{~nm}$ ). 

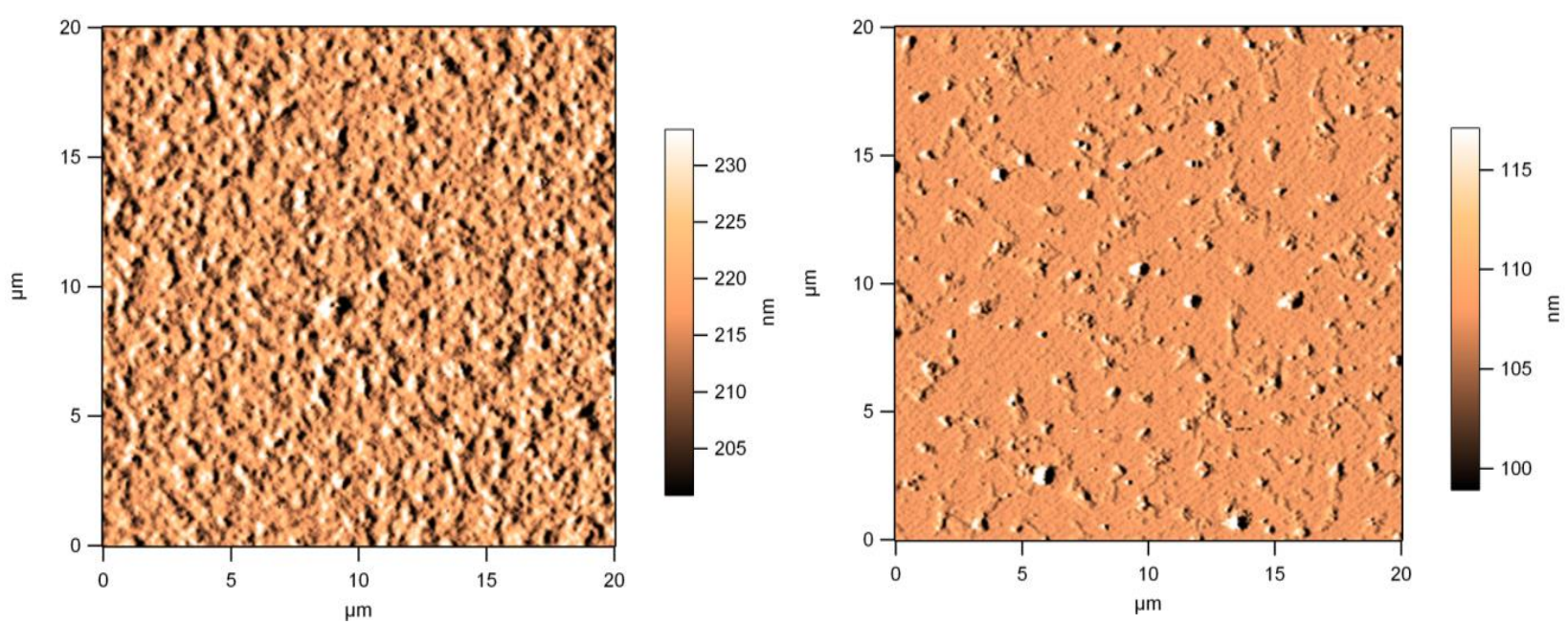

Figure 2.5: The atomic force microscopy pictures taken for thickness of $2 \mu \mathrm{m}$ (left) and thin 100nm (right) layers used in P3HT: PCBM organic solar cells.

It is evident that the morphology of the thicker sample shows a higher roughness (roughness $=60 \mathrm{Nm}$ ) compared to that of the thinner sample (roughness $40 \mathrm{Nm}$ ) on the right. The particles were less agglomerated in the thinner sample which was important to obtain a more uniform film.

\subsubsection{Absorbance measurements of organic solar cells}

Another important characterization of these solar cells is the absorbance measurement done using UV-VIS spectroscopy. Absorbance measurement describes a material property that chooses which wavelengths in the region are light sensitive. First the incident and transmission power through the sample is measured by a Silicon photodetector (Thor Lab.). Then the transmission of light is measured through a glass slide which the sample is deposited. These values will be subtracted from the transmission values when the sample is present in order to avoid the reflection by the 
glass. Then the following Beer-Lambert law is used to calculate the absorption coefficient of the organic polymers.

$$
I_{1}=I_{0} e^{-\propto x}
$$

Where $I_{1}$ the power of is transmitted light, $I_{0}$ power of incident light, $\mathrm{x}$ is the sample thickness and $\propto$ is the absorption coefficient $\left(\mathrm{cm}^{-1}\right)$. Here the interference effects and light scattering effects by the sample surface are neglected.

The mixture of P3HT: PCBM blend solution was spin coated on a cleaned glass slide with a weight ratio of $5: 4(10 \mathrm{mg} / 8 \mathrm{mg}$ in chlorobenzene). Its absorbance spectrum is shown in Figure 2.6.

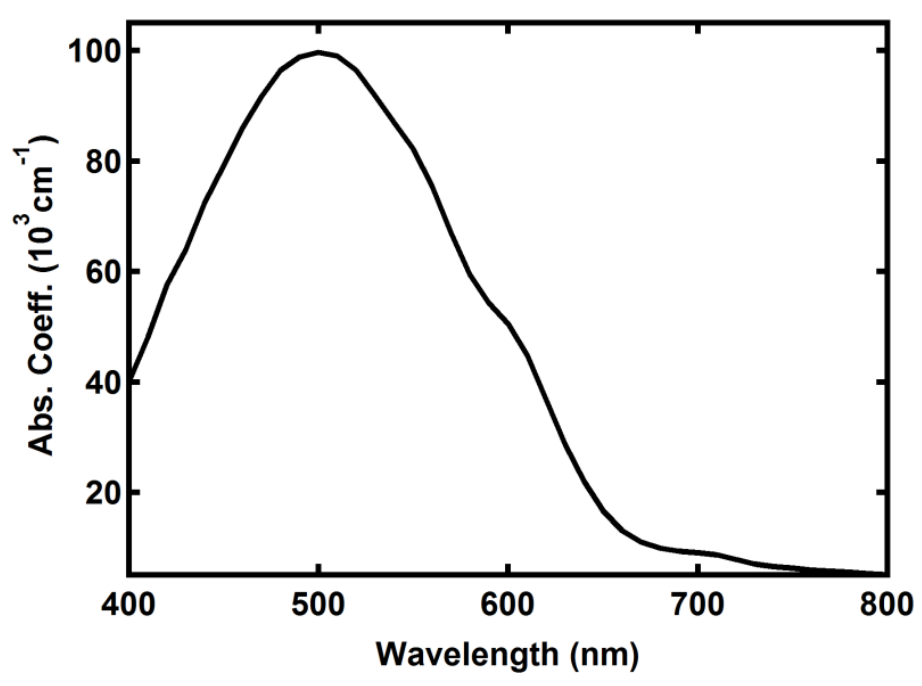

Figure 2.6: Absorption coefficient spectrum of P3HT: PCBM active layer measured using UV-VIS spectrometer.

The absorbance by the material started at $650 \mathrm{~nm}(1.9 \mathrm{eV})$ and maximized at around 510nm[59] which describes the optical band gap of P3HT. The absorbance peak corresponds to singlet state $\left(S_{1}\right)$ was seen at around $700 \mathrm{~nm}$. Next graph (Figure 2.7) 
shows the absorbance of MDMO-PPV: PCBM (10:40mg/ml in 1,2Dicholorbenzene) sample along with different annealing temperatures.

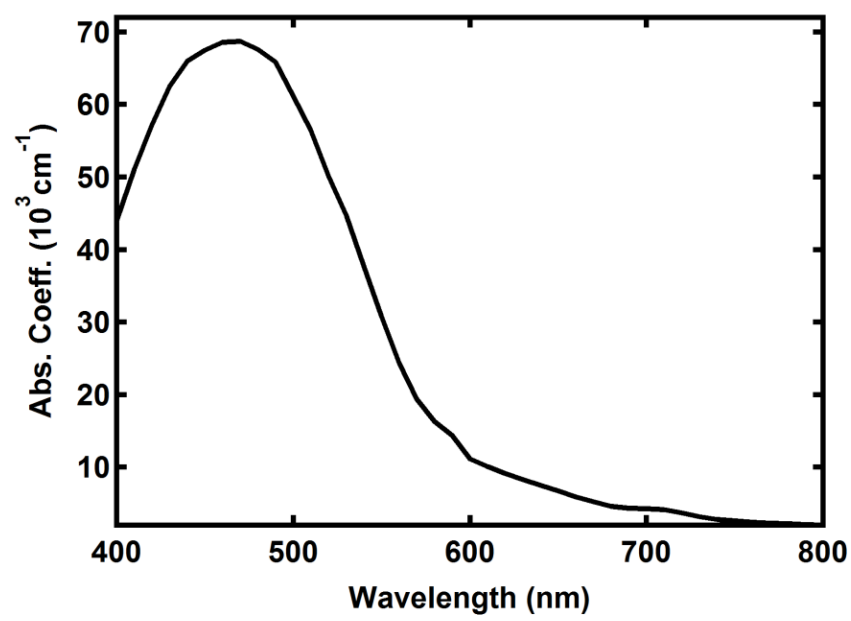

Figure 2.7: Absorption coefficient spectrum of MDMO-PPV: PCBM active layer measured using UV-VIS spectrometer.

Here, the absorbance maximized at around 470nm[59, 60] with a higher shoulder observing at $700 \mathrm{~nm}$ PCBM peak. This outcome occurred as a result of the higher composition of PCBM in the active blend. The absorbance of the MDMO-PPV: PCBM solar cells of 1:4 composition was done for different annealed temperatures (Figure 2.7). These results clearly demonstrate that annealing to a higher temperature increases the absorbance by the material. 


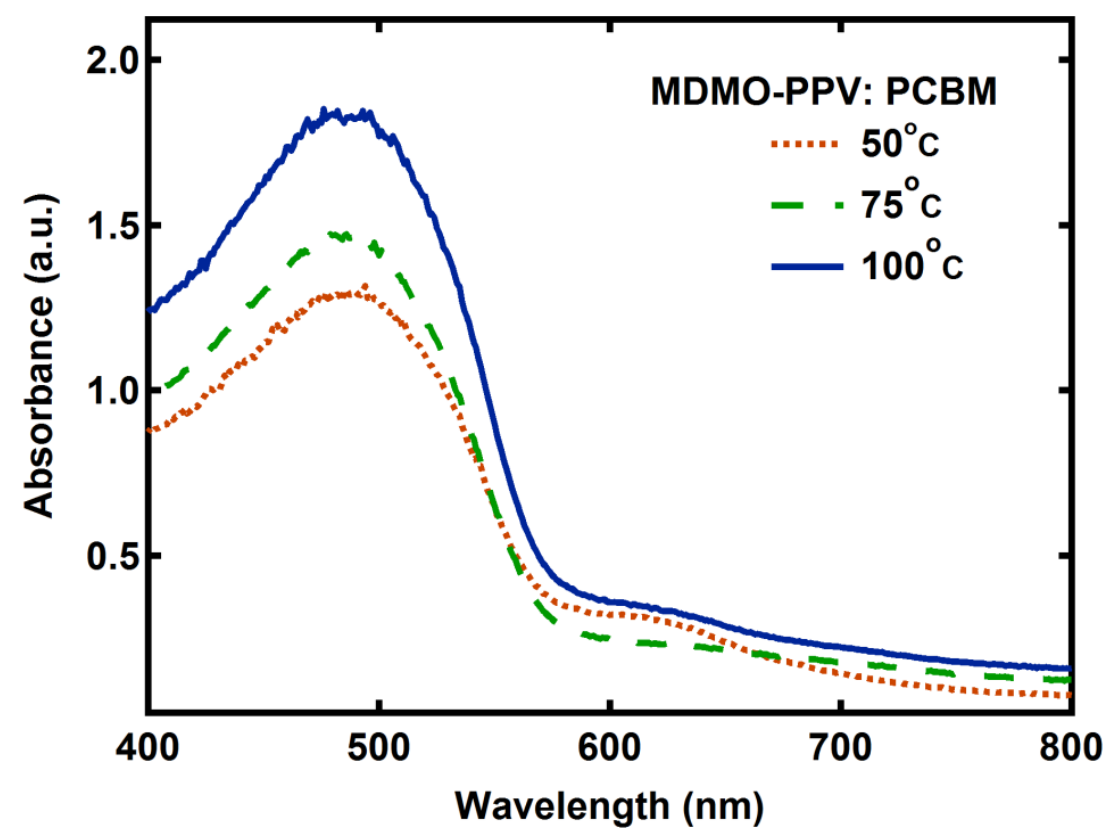

Figure 2.8: Absorbance spectrum of MDMO-PPV: PCBM active layers annealed at different temperatures measured using UV-VIS spectrometer.

The increase in the absorbance by the material with elevated annealed devices mainly occurred due to improvement of the charge transfer by crystallization of the domains. This improvement in crystallization is ultimately limited by the structure deformation occurred at annealing temperature around $150^{\circ} \mathrm{C}$. 


\subsection{Photoluminescence spectra of organic solar cells}
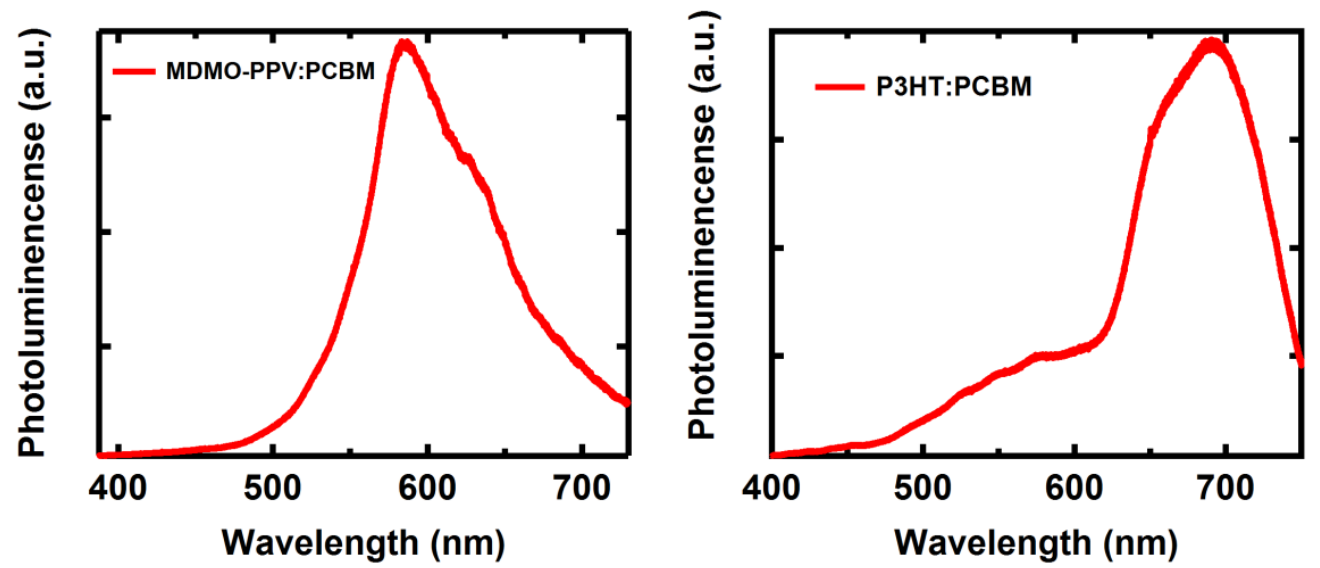

Figure 2.9: Photoluminescence spectra of MDMO-PPV: PCBM and P3HT: PCBM active layers measured using $325 \mathrm{He}-\mathrm{Cd}$ excitation source.

Photoluminescence represents a radiative recombination of electron in LUMO level with a hole in HOMO level. This energy transition represents the minimum band edge prior to recombination. The photoluminescence of the P3HT: PCBM solar cell was conducted using 325nm excitation from a He-Cd laser using a Renishaw RL633 spectrometer. As typically observed, there was a Stokes shift between the absorbance and luminescence spectra due to the thermalization of absorbed carriers to the lower energy states near the band edge prior to radiative recombination[61]. 


\subsection{J-V characterization of organic solar cells}

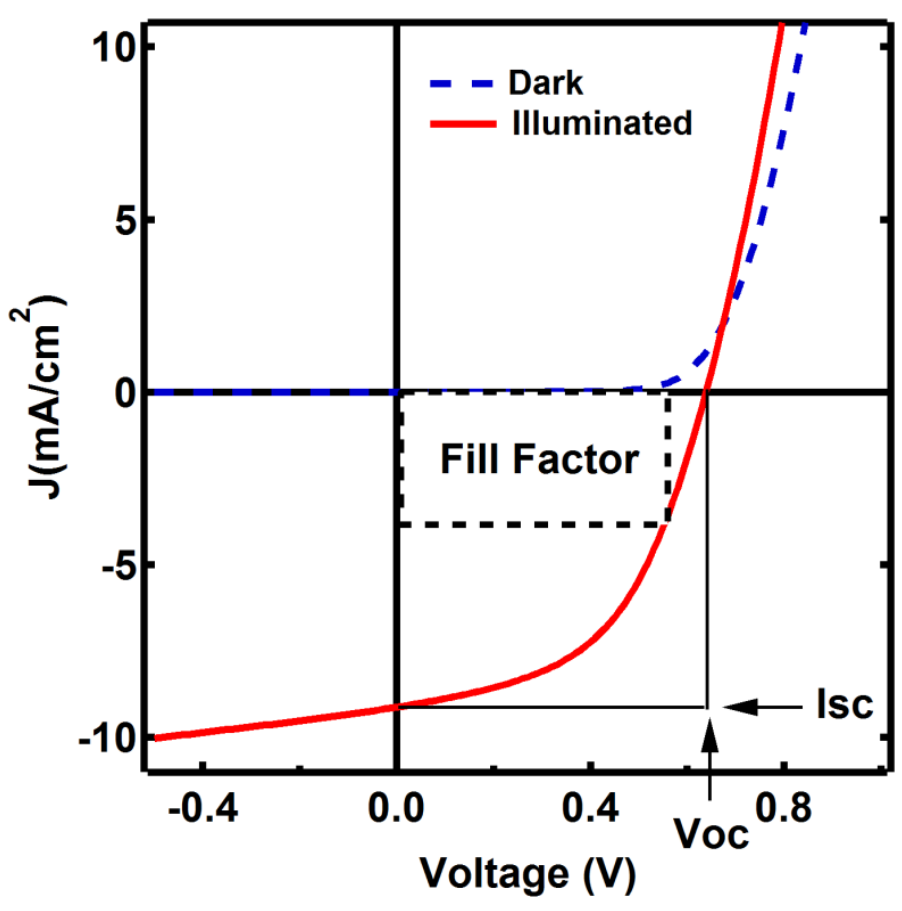

Figure 2.10: J-V characteristic of thickness of 100nm P3HT: PCBM organic solar cell measured at AM1.5G solar simulator.

A typical $\mathrm{J}-\mathrm{V}$ characterization of a P3HT: PCBM solar cell under AM1.5G conditions shown in Figure 2.10. The dash line represents the dark current while solid line represents the current under illumination. The curve total current at short circuit conditions was the short circuit current $\left(9.2 \mathrm{~mA} / \mathrm{cm}^{2}\right)$ and open circuit voltage was about $0.64 \mathrm{~V}$ that gives the fill factor of 0.5 and overall efficiency of about $2.93 \%$. The fill factor and efficiency $(\eta)$ were calculated using the standard equations cited in C. Winder et al[58].

The comparison of J-V characteristic of P3HT: PCBM solar cells of two different thickness devices are shown in the Figure 2.11 

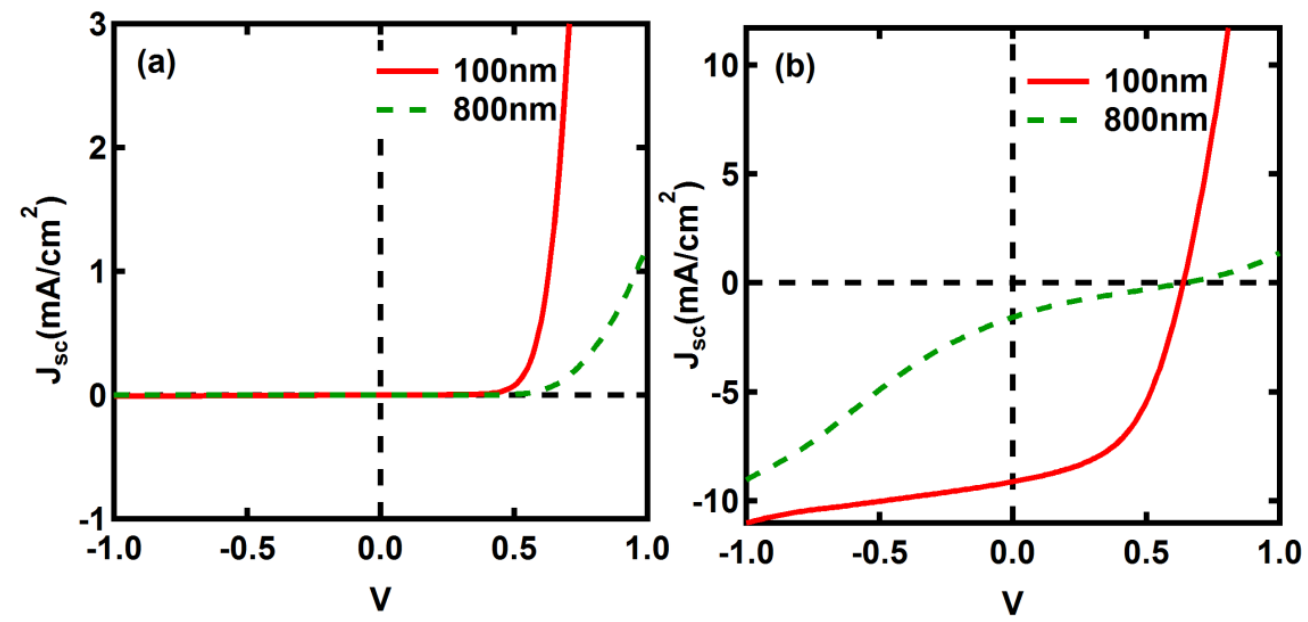

Figure 2.11: J-V characteristic of thickness of 100nm and 800nm of P3HT: PCBM organic solar cells measured at AM1.5G solar simulator. The dark currents are shown in the figure (a) and characteristics under illumination are shown in figure (b).

Both thin and thick devices show almost the same open circuit voltage of $\sim 0.64 \mathrm{~V}$ while the short circuit current of thick device $(800 \mathrm{~nm})$ is reduced to $\sim 2 \mathrm{mAcm}^{-2}$. Also the series resistance of $20 \Omega \mathrm{cm}^{2}$ for thin device is increased to approximately $250 \Omega \mathrm{cm}^{2}$. In ideal case, series resistance should be zero to achieve higher fill factors and efficiencies of organic solar cells. Furthermore the reduction of short circuit current in thick devices might be mainly due to reduced charge transfer effects by high density of molecules in a poor crystal formation. Similar J-V characteristics have been obtained for MDMO-PPV: PCBM solar cells and the results are summarized in the Table 2.1. 


\begin{tabular}{|c|c|c|c|c|}
\hline Solar Cell device & $\mathrm{J}_{\mathrm{sc}}\left(\mathrm{mA} / \mathrm{cm}^{2}\right)$ & $\mathrm{V}_{\mathrm{OC}}(\mathrm{V})$ & FF & $\eta(\%)$ \\
\hline P3HT:PCBM (100 nm) & 9.14 & 0.64 & 0.50 & 2.93 \\
\hline P3HT:PCBM (800 nm) & 1.58 & 0.64 & 0.18 & 0.19 \\
\hline MDMO-PPV:PCBM & 0.14 & 0.79 & 0.23 & 0.03 \\
$(100 \mathrm{~nm})$ & 0.06 & 0.19 & 0.24 & 0.003 \\
\hline MDMO-PPV:PCBM (2 & & & & \\
\hline
\end{tabular}

Table 2.1: Summary of J-V characteristics of MDMO-PPV and P3HT organic solar cells for thick and thin samples.

\subsection{Mobility measurements of P3HT: PCBM solar cells (Time of flight technique)}

The time of flight technique is used to measure the mobility of organic solar cells. This method basically measure the time for the carriers to travel from front electrode to the counter electrodes which is in micro seconds. The excess carriers are generated by high power light pulse incident on ITO electrode. A voltage is applied to ITO to create a potential difference between the electrodes so that the photoinduced carriers flow to the respective electrodes.

The samples were made following similar fabrication steps described in chapter 1 to prepare organic solar cells bulk heterojunction structures, except, that the samples were drop casted to make it thick $(\sim \mu \mathrm{m})$. The circuit was connected according to the time of flight measurement setup discussed in Chapter 1 Figure 1.6. To apply the DC voltage, a constant battery pack with output voltage of $38 \mathrm{~V}$ was used. The laser at $511 \mathrm{~nm}$ was 
used as the excitation source with a power of $4 \mathrm{~mW}$ output from OPA. This high intense light is illuminated at front side (ITO) of the device. Positive or negative voltage of $38 \mathrm{~V}$ was applied to the front side of the cell, and the back side (Al) was connected either to current amplifier or directly to a digital oscilloscope. A buffer resistor was used to prevent high current flowing into the scope. The buffer resistor value was $2.1 \mathrm{KOhms}$ (refer setup). According to the polarity of applied voltage, carrier type was determined. If positive bias was applied to ITO side, holes charge mobility was measured and for electrons, negative bias must be applied[49]. When the short laser pulse was applied to the device, a charge flow occurred from the ITO side to the aluminum which was detected by the oscilloscope as a transient decay curve. The width of the light pulse and the repetition rate of the laser pulse determine the total decay time of this transient current obtained from the device. All the transient measurements were measured up to 1ms. Digital output files of the transient decay curves were collected by the Agilent DSO1000 series software. Transient measurements were plotted in log-log scale in Igor for different concentrations to examine the dependence. Thicknesses of the samples were measured again by dektek profilometer. 


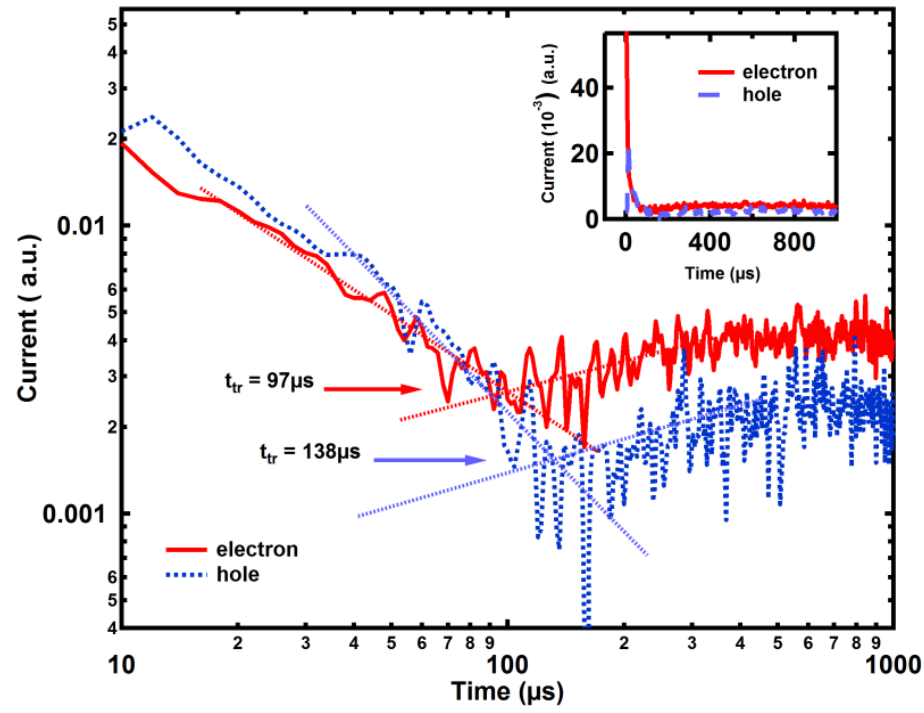

Figure 2.12: The log-log transient current measurements for P3HT: PCBM sample of 5:4 $\mathrm{wt} \%$. (inset): the transient current measurement of electron and hole for the same sample of P3HT: PCBM.

Calculation of the electron and holes mobilities were done using the equation below, where $\mathrm{d}$ is the active device thickness, $\mathrm{V}$ is the applied voltage and $\mathrm{t}_{\mathrm{tr}}$ is the transit time.

$$
\mu=\frac{d^{2}}{V \cdot t_{t r}}
$$

[49]According to transient measurement in graph 2.12, $\mathrm{t}_{\mathrm{tr}}=97 \mu \mathrm{s}$ and $\mathrm{t}_{\mathrm{tr}}=138 \mu \mathrm{s}$ were obtained for electrons and holes respectively. Device thickness for this particular device was measured as $0.859 \mu \mathrm{m}$ and $V=38 \mathrm{~V}$. Substituting into Equation 4.1, we obtained $\mu_{\mathrm{n}}$ $=2.0 \times 10^{-4} \mathrm{~cm}^{2} / \mathrm{Vs}$ and $\mu_{\mathrm{h}}=1.4 \times 10^{-4} \mathrm{~cm}^{2} / \mathrm{Vs}$. These values are similar to the mobility values obtained in literature[62]. Similar time of flight measurements were conducted for other compositions (1:2, 5:1 and 10:1) of P3HT: PCBM samples (figure 2.13). 

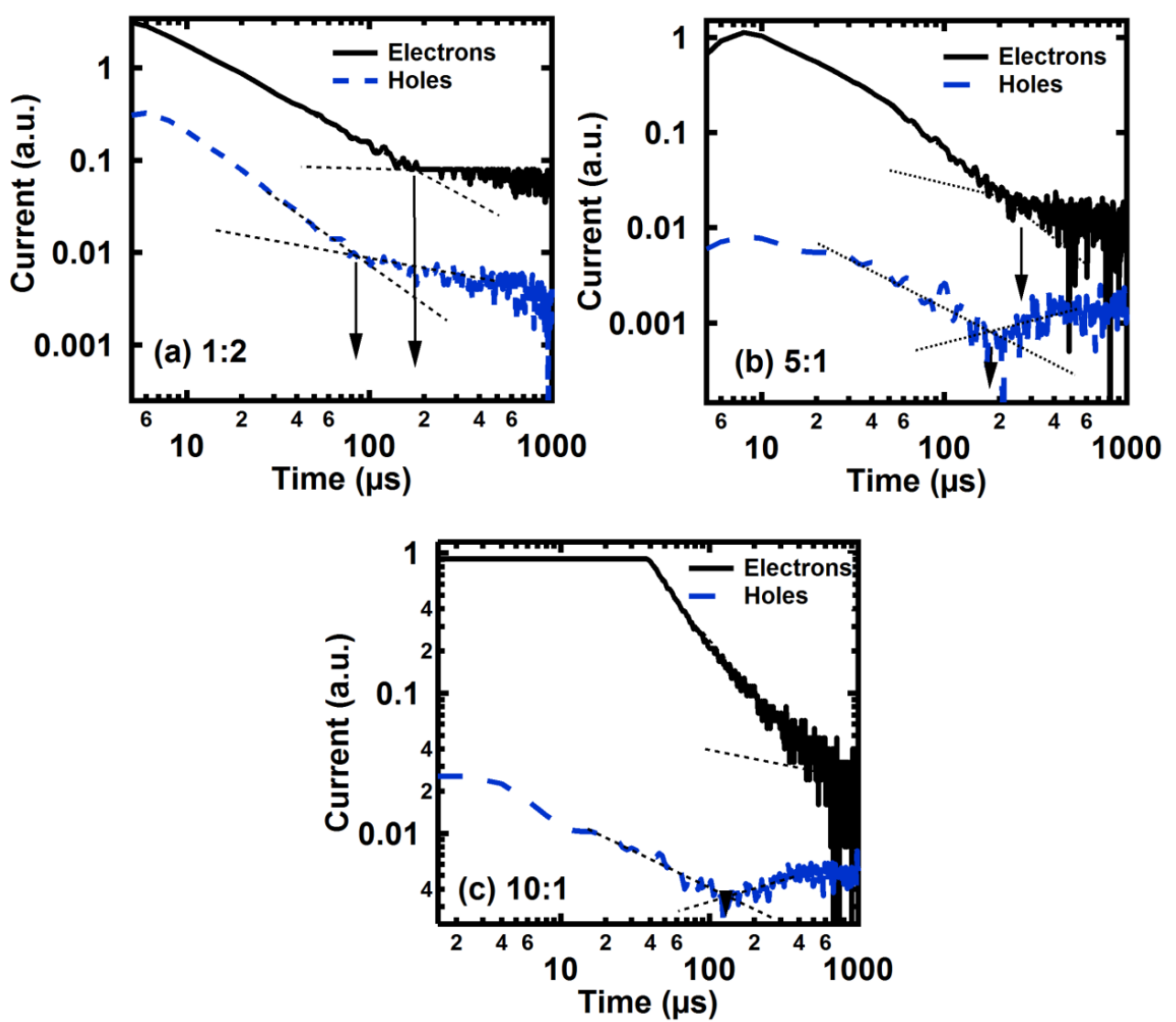

Figure 2.13: The log-log transient current measurements for P3HT: PCBM sample of (a)

$$
\text { 1:2 (b) 5:1 and (c) 10:1. }
$$

The transit times of these transient plots and the corresponding electron and hole mobilities values were calculated using Equation 4.1. Summarized results of these calculations are given in the Table 2.2. For all these mobility measurements of the different weight ratios, the experimental parameters such as applied voltage and power of the laser light were kept constant. The thickness of the sample kept constant $(2 \mu \mathrm{m})$ to avoid the fluctuations from sample to sample. 


\begin{tabular}{|c|c|c|c|c|}
\hline Weight ratio & $\begin{array}{c}\text { Electron } t_{\text {tr }} \\
(\mu \mathrm{s})\end{array}$ & $\begin{array}{c}\text { Holes } \mathrm{t}_{\mathrm{tr}} \\
(\mu \mathrm{s})\end{array}$ & $\begin{array}{c}\text { Electron mobility } \\
\left(\mu_{\mathrm{n}}\right) \\
\left(\mathrm{cm}^{2} / \mathrm{Vs}\right)\end{array}$ & $\begin{array}{c}\text { Holes mobility } \\
\left(\mu_{\mathrm{h}}\right) \\
\left(\mathrm{cm}^{2} / \mathrm{Vs}\right)\end{array}$ \\
\hline $1: 4$ & 12 & 20 & $8.42 \times 10^{-5}$ & $5.05 \times 10^{-5}$ \\
\hline $1: 2$ & 180 & 88 & $5.62 \times 10^{-5}$ & $1.15 \times 10^{-5}$ \\
\hline $5: 1$ & 230 & 182 & $4.39 \times 10^{-6}$ & $5.55 \times 10^{-6}$ \\
\hline $10: 1$ & 232 & 182 & $4.35 \times 10^{-6}$ & $5.55 \times 10^{-6}$ \\
\hline
\end{tabular}

Table 2.2: The summarize results of mobilities of P3HT: PCBM samples for different weight ratios.

According to the Table 2.2, the highest electron mobility is given for the samples of 1:4 weight ratio. Smallest mobilities are given for the samples with 10:1 weight ratio. This important feature matched with charge collection lengths in the model which will be discussed in next chapter.

\subsection{Initial photocurrent measurements on organic blends}

The photocurrent measurements of an organic solar cell is the result of many photophysical processes occurred in the bulk as well as in the interface between the donor acceptor interfaces. Direct current (DC) photocurrent spectra can be used to study all of these charge dissociations, charge transport and recombination processes. This section describes an initial comparison of an active layer thickness dependence on the optical 
absorbance and the photocurrent of these blends. Figure 2.14 demonstrates the absorbance of thin and thick devices of P3HT: PCBM and MDMO-PPV: PCBM. The fraction of solar photon flux is also shown.
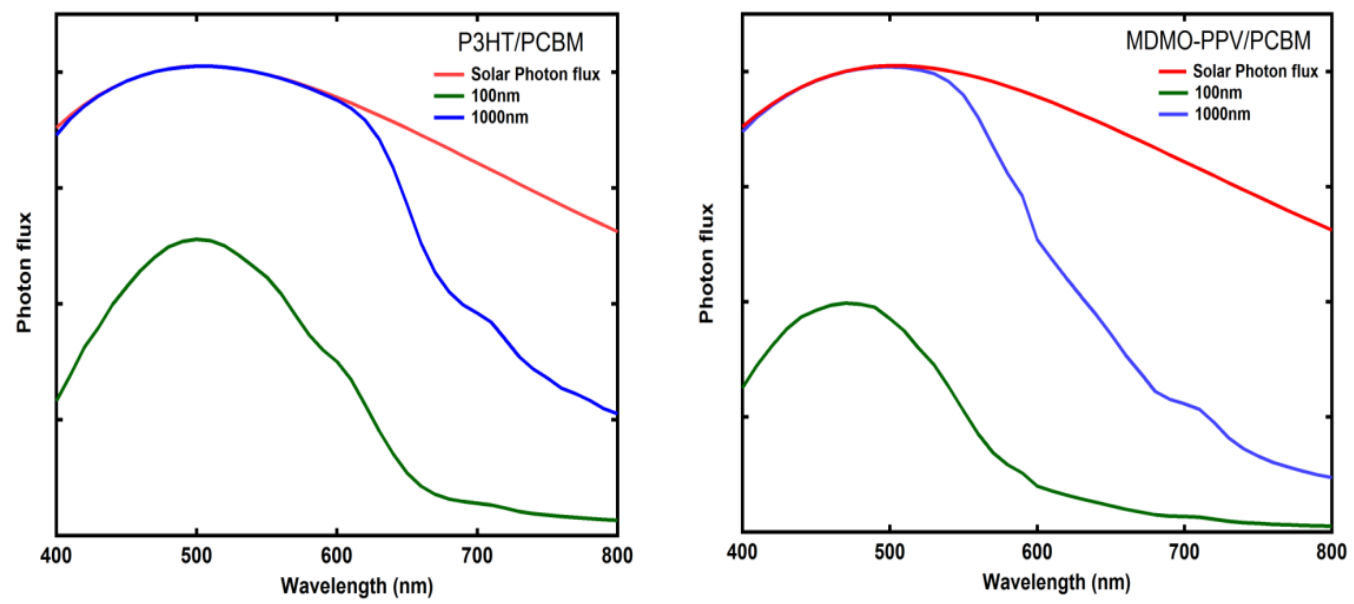

Figure 2.14: Absorption percentage of P3HT: PCBM and MDMO-PPV: PCBM active layers of thick and thin compared to solar photon flux.

Same thickness samples were used for both blends of P3HT: PCBM and MDMOPPV: PCBM. Increasing the thickness of samples from $100 \mathrm{~nm}$ to $1 \mu \mathrm{m}$ increases the absorbance of the photon flux. This indicates that the exciton generation has a linear dependence on active layer thickness in both materials. Figure 2.15 shows the integrated photocurrent obtained for P3HT: PCBM solar cells with different active layer thicknesses. 


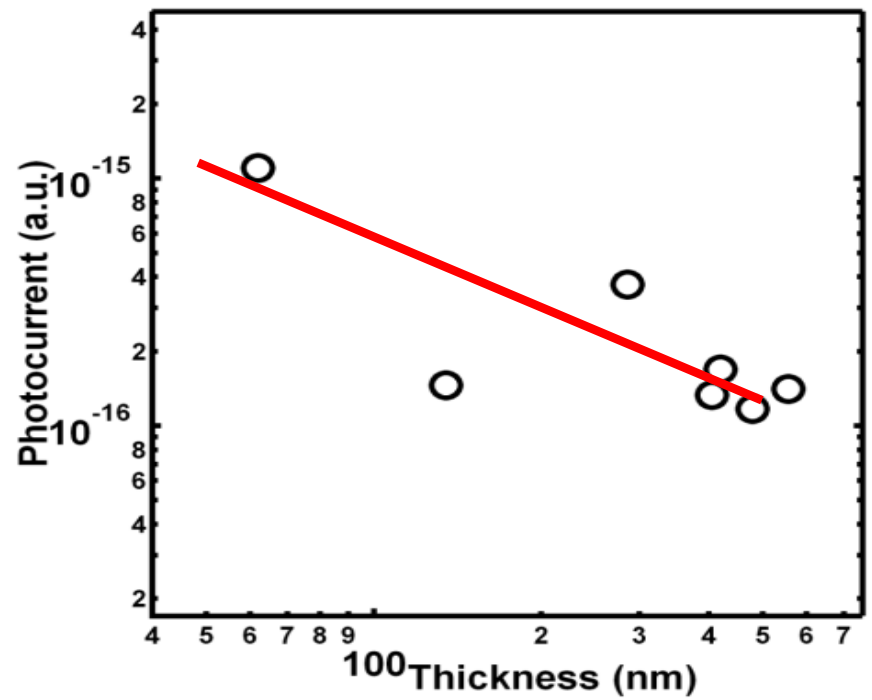

Figure 2.15: Photocurrent vs. thickness dependence of P3HT: PCBM organic solar cell. When the thickness of the active layer increases the total photocurrent decreases. This means that the photocurrent generation by these organic blend materials do not exclusively depends on the photo-absorption, but also relies on other mechanisms that govern the photocurrent. Hence the rest of this work is mainly devoted to the objective of understanding the exact mechanism of photocurrent dependence on thickness of organic BHJs and possible ways to enhance the efficiencies. 


\section{CHAPTER III: CHARGE EXTRACTION MODEL}

This chapter discusses the charge extraction model used to understand the experimental observations in photocurrent variation on thickness of organic solar cells. The first part discusses the derivation of the model in general and then the fraction of charge extractions for different conditions is discussed. Finally the difference of front and back illuminations along with the model calculations are discussed.

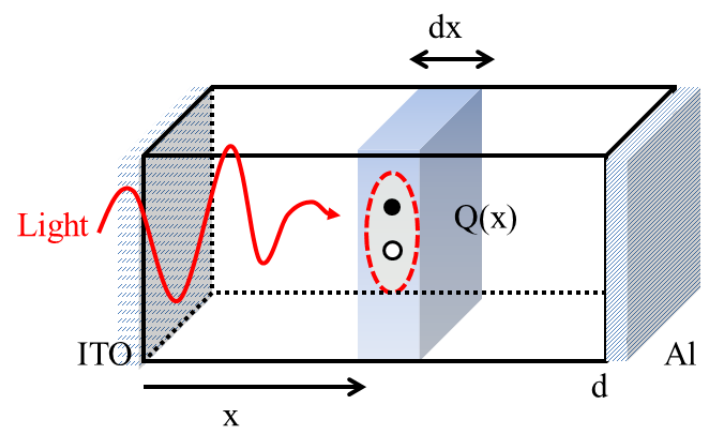

Figure 3.1: Charge $\mathrm{Q}(\mathrm{x})$, generated at a distance $\mathrm{x}$ from the front side of the active layer upon light absorption.

Consider an organic solar cell with an active layer thickness of $\mathrm{d}$ as shown in figure 3.1. When light illuminates at the front surface (ITO electrode), it penetrates into the active device due to optically transparent ( $100 \%$ in visible region of the spectrum) 
indium tin oxide electrode. Assume there will be $\mathrm{Q}(\mathrm{x})$ amount of charge generated due to photon absorption at an arbitrary distance $\mathrm{x}$ from the front contact. This excitons pair diffuse to a donor-acceptor (D-A) interface and at the interface they dissociate. After dissociating, electron move to the electron acceptor (fullerene) and holes will be in electron donor (conjugated polymer) creating the charge transfer excitons (CTE) (this is still bound by high binding energies but transferred to acceptor). Once this pair dissociates by the built-in electric field of the work function difference, they become free carriers which eventually move to the respective electrodes. This charge will be collected by the holes collecting electrode and by the electron collecting electrode. As most semiconductors, the organic bulk hetero junctions made out by P3HT: PCBM has a variation of the absorption profile within the visible region of spectrum.

\subsection{Photoinduced charge generation in semiconductors}

The penetration depth relates to the absorption profile of a material and photon energy of the light. The absorption profile is described by a constant absorption coefficient denoted by $\alpha$. This basically explains how the light intensity attenuated when passing through a material. 

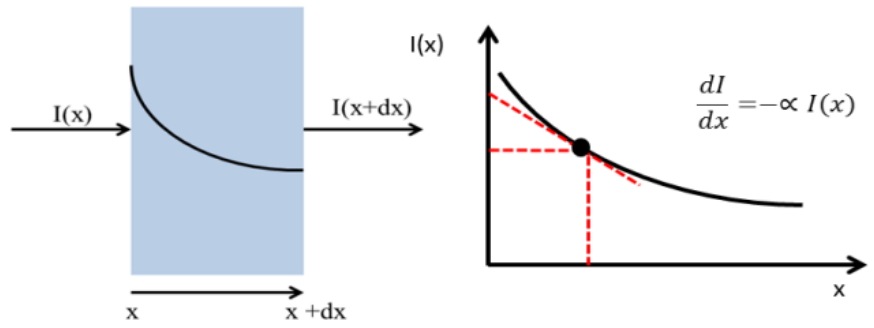

Figure 3.2: Attenuation of light in a slab of thickness $\mathrm{x}$ and light intensity decay as a function of absorption coefficient.

Suppose a beam of photons of intensity $\mathrm{I}_{0}$ incident normally per unit area per second on an absorbing material $\alpha(\mathrm{E})$.

And the intensity of the incident beam at a distance $\mathrm{x}$ from the point of incidence per unit area per unit time is $\mathrm{I}(\mathrm{x})$, given by

$$
\frac{d(I(x))}{d x}=-\propto I(x)
$$

Where, $\alpha$ is the absorption coefficient $\left(\mathrm{cm}^{-1}\right)$. Assuming each photon absorbed on the material creates one electron-hole pair (EHP), the number of photons absorbed per unit area at one second within a distance of $d x$ is given by $\mathrm{I}(\mathrm{x}+\mathrm{dx})-\mathrm{I}(\mathrm{x})$. Hence one can write

$$
\begin{aligned}
& \frac{I(x+d x)-I(x)}{d x}=-\propto I(x) \\
& \frac{\text { No.of EHPs }}{\text { Volume }}=\frac{I(x)-I(x+d x)}{d x}=\propto I(x)
\end{aligned}
$$


by taking the initial incidence light intensity as $\mathrm{I}_{0}$ and applying the boundary conditions of $\mathrm{I}(\mathrm{x}=0)=\mathrm{I}_{0}$, the solution to equation 3.1 given by

$$
\begin{gathered}
I(x)=I_{0} e^{-\propto x} \\
\text { Then } I(x)-I(x+d x)=\propto I_{0} e^{-\propto x} d x
\end{gathered}
$$

Equation 3.5 describes the amount of light absorbed when travelling through the material of absorption property $\alpha$ within a distance of $\mathrm{dx}$. Assume all this light absorbed creates electron-hole pair (EHP). Then the total electron charge $n(x)$ and holes charge $p(x)$ given by

$$
\begin{aligned}
& n(x)=\frac{\propto}{\tau_{e}} I_{0} e^{-\propto x} \\
& p(x)=\frac{\propto}{\tau_{h}} I_{0} e^{-\propto x}
\end{aligned}
$$

Where $\mathrm{I}_{0}$ is the initial total light intensity, $\alpha$ is the absorption coefficient of the material, $\tau_{\mathrm{e}}, \tau_{\mathrm{h}}$ are the electron and hole carrier lifetimes and $\mathrm{x}$ is arbitrary distance from the front contact.

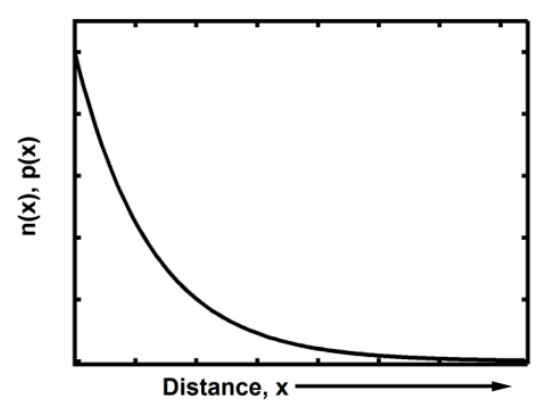

Figure 3.3: Decay of charge generated along the distance of the active layer. 
The graph 3.3 shows the decay of total charge along the distance of the medium $\alpha\left(\lambda=\lambda_{0}\right)$.

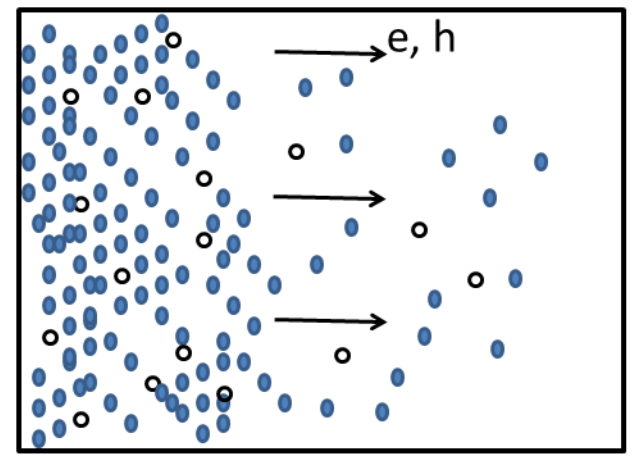

Diffusion

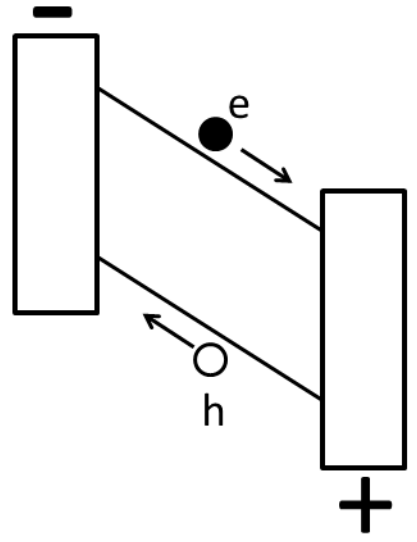

Drift

Figure 3.4: Diffusion and Drift contributions in a semiconductor medium. In diffusion, both electrons and holes travel from high concentration to low concentration while in drift the electrons travel from high potential to low and holes travels from low potential to high potential.

Consider the two basic processes of current conduction in semiconductors. They are drift due to an electric field within the semiconductor and the diffusion due to carrier concentration gradient (Figure 3.4). Because of absorption variation of the material, carrier generation becomes non-uniform creating e-h concentration variation with respect to the distance along the sample. And carriers tend to move from high concentration to low which is called the diffusion. The drift occurs because of different work function materials creating an electrical potential difference. Charge transport occurs by the combination of these two processes which is described by the equation given by 


$$
\begin{aligned}
& J_{n}(x)=q \mu_{e} n(x) \varepsilon(x)+q D_{e} \frac{d(n(x))}{d x} \\
& J_{p}(x)=q \mu_{h} p(x) \varepsilon(x)-q D_{h} \frac{d(p(x))}{d x}
\end{aligned}
$$

Here $\mathrm{q}$ is the charge of the carrier, $\mu_{e}, \mu_{h}$ electron and hole mobilities and $\mathrm{n}(\mathrm{x}), \mathrm{p}(\mathrm{x})$ are electron and hole carrier densities. $\varepsilon$ is the drift electric field caused by the work function difference between the $\mathrm{Al}$ and ITO electrodes. $\mathrm{D}_{\mathrm{n}}$ is the diffusion constant given by the Einstein relation[25]

$$
D_{n}=\frac{k T}{q} \mu_{n}
$$

Plugging 3.6 and 3.7 into continuity Equation 3.8 and 3.9, one can write the drift and diffusion components as

$$
\begin{array}{r}
J_{\text {drift }}(x)=q \mu \frac{\alpha}{\tau} I_{o} e^{-\propto x} \varepsilon(x) \\
J_{\text {diffusion }}(x)=-q D \frac{\alpha^{2}}{\tau} I_{o} e^{-\propto x}
\end{array}
$$

Now taking the ratio of 3.11 to 3.12 ,

$$
\frac{J_{\text {drift }}}{J_{\text {diffusion }}}=\frac{\mu \varepsilon}{\alpha D}
$$

Substituting 3.10 into 3.13,

$$
\frac{J_{\text {drift }}}{J_{\text {diffusion }}}=\frac{\varepsilon q}{\alpha \mathrm{KT}}
$$


Since holes created moves towards ITO and electrons move towards Al. This occurs mainly due to the work function alignment of ITO and Al. ITO has a work function of 4.7 $\mathrm{eV}$ and $\mathrm{Al}$ of $4.1 \mathrm{eV}[7,63]$ with respect to the vacuum energy. Hence the built-in electric field will occurred from ITO to Al inside the material.

By taking $\mathrm{T}=300 \mathrm{~K}$ and $\mathrm{K}=1.3806488 \times 10^{-23} \mathrm{~m}^{2} \mathrm{~kg} \mathrm{~s}^{-2} \mathrm{~K}^{-1}$ ratio of 3.14 per unit charge gives

$$
\frac{J_{\text {drift }}}{J_{\text {diffusion }}}=\frac{0.6 q}{\alpha(0.0256) \cdot \mathrm{d}}=\frac{23.4375}{\propto . d}
$$

The ratio of drift to diffusion at different absorption coefficients as a function of thickness $d$ is shown in the graph of 3.5 calculated using the Equation 3.15.

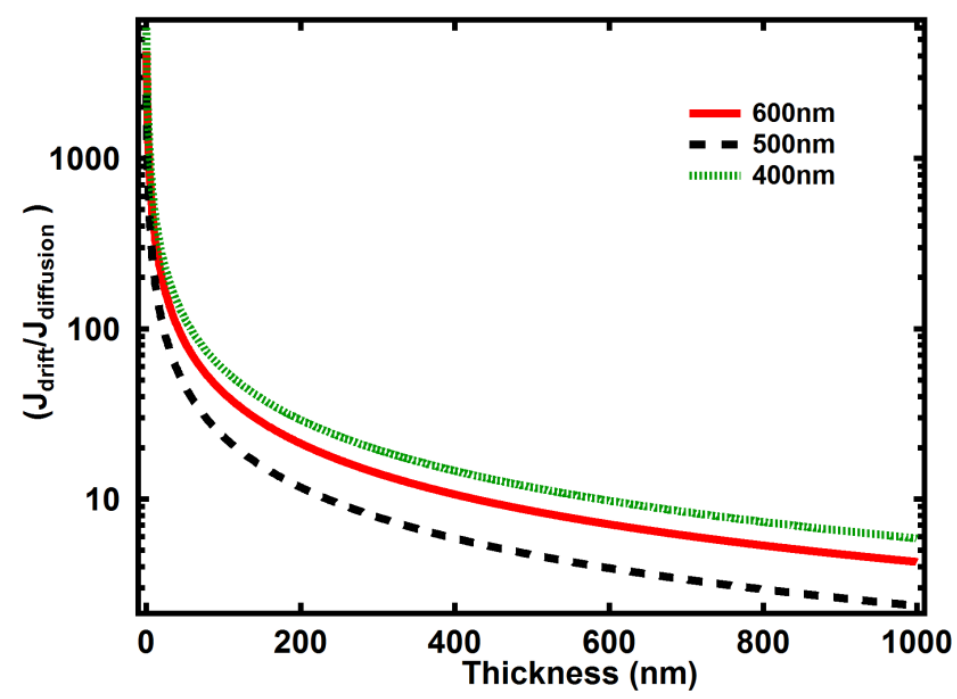

Figure 3.5: Drift and Diffusion components of decay per unit charge along the distance at the wavelengths of 600,500 and $400 \mathrm{~nm}$.

According to the graph, the drift contribution for the charge generation is about 10 times higher to diffusion contribution for a sample thickness of 400nm. Hence the main 
contribution of the equations 3.8 and 3.9 comes from the drift contribution for thin devices. But when thickness is increasing the diffusion term becomes non-negligible. For this model we neglected the diffusion term assuming all charge carriers transport via the drift contribution caused by the electric field.

\subsection{Derivation of the charge extraction model}

\subsubsection{Charge collections in model derivation for front illumination}

This section introduces a term $d_{i}$ called as charge collection length $\left(d_{e}\right.$ for electrons collection length and $d_{h}$ for holes collection length). They are defined as the collection lengths travel to the electrodes before recombined. As shown in the Figure 3.6, due to recombination the amount of holes collected by the front contact is assumed to drop-off exponentially with distance, so the holes collected charge can be written as

$$
Q_{h}(x)=q \propto I_{o} \exp (-\propto x) \cdot \exp \left(-\frac{x}{d_{h}}\right)
$$

Where $\mathrm{d}_{\mathrm{h}}$ is the characteristics collection length for holes. Set $q I_{o}=Q_{o}$, and above 3.16 becomes

$$
Q_{h}(x)=\propto Q_{o} \exp (-\propto x) \cdot \exp \left(-\frac{x}{d_{h}}\right)
$$

Similarly for electrons

$$
Q_{e}(x)=\propto Q_{o} \exp (-\propto x) \cdot \exp \left(-\frac{(d-x)}{d_{e}}\right)
$$




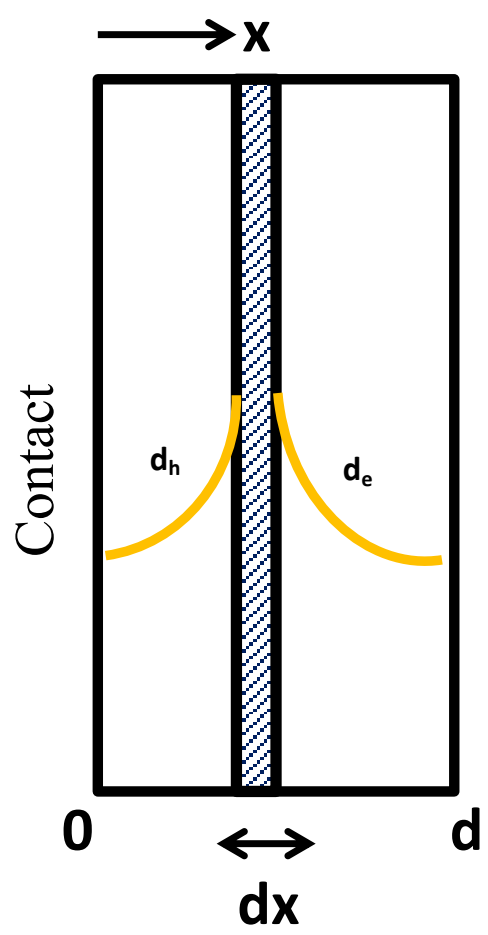

Figure 3.6: Schematic of the charge collection lengths for electrons and holes. Charge collection will be reduced exponentially compared to the number generated due to recombination.

The surface recombination of the free carriers at the metal-semiconductor interface must not be neglected specially for polymeric semiconductors which the trap states are possible. When this model applies to amorphous organic solids or polymers, it is important to recognize that the dominance of intra-molecular forces create tightly bound excitons which are not separated by the electric fields due to difference in work function. As described in the introduction of this dissertation, the excitons diffuse towards the donor-acceptor (D-A) junction dissociate only at the interface creating free carriers in donor and acceptor phase. Once they are dissociated, they are approximated to act as free 
carriers. These free carriers are assumed to be moved by the constant E-field due to work function difference of the carrier collecting electrodes.

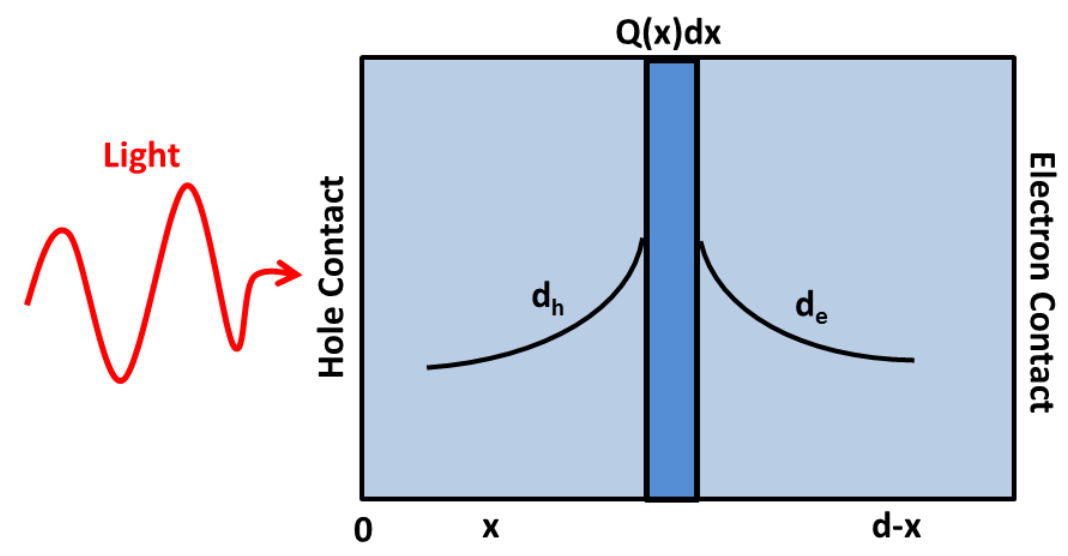

Figure 3.7: Generation of population of charge $\mathrm{Q}(\mathrm{x})$ at a distance $\mathrm{dx}$ from the front contact decays exponentially due to recombination. Here the light illuminates from front contact.

The charge collected from the front electrode is given by Equation 3.16. The collection lengths $\left(d_{e}\right.$ and $\left.d_{h}\right)$ decay exponentially when they move from the charge generated position to the respective electrodes by recombining with neighboring free electron and holes. Hence $e^{-x / d_{h}}$ is introduced in equation 3.17.

$$
\begin{gathered}
Q_{h}=\int_{0}^{Q_{h}} d Q_{h}(x)=\int_{0}^{d} Q_{0} \alpha e^{-\alpha x} e^{-x / d_{h}} d x \\
\frac{Q_{h}}{Q_{0}}=\frac{\alpha}{\left(\alpha+\frac{1}{d_{h}}\right)}\left[1-e^{-d\left(\alpha+\frac{1}{d_{h}}\right)}\right]
\end{gathered}
$$


Similarly for collected electrons from the back contact is given by

$$
\begin{gathered}
Q_{e}=\int_{0}^{Q_{e}} d Q_{e}(x)=\int_{d}^{0} Q_{0} \alpha e^{-\alpha x} e^{-(d-x) / d_{e}} d x \\
\frac{Q_{e}}{Q_{0}}=\frac{\propto e^{-\left(d / d_{e}\right)}}{\left(\frac{1}{d_{e}}-\propto\right)}\left[e^{d\left(\frac{1}{d_{e}}-\propto\right)}-1\right]
\end{gathered}
$$

This description is valid for assuming the charge dissociated from the excitonic state, travels to the respective electrodes in which electrons travel to front contact and holes travel to back contact. It is suggested that this charge transport is the most dominant assuming negligible leakage currents.

\subsubsection{Dependence of Charge extraction on absorption coefficient for P3HT and MDMO-PPV solar cells}

The model calculations are done to obtain the fractions of electron and hole charges extracted from the electrodes for the samples of P3HT: PCBM and MDMO-PPV: PCBM solar cells varying sample thickness using the equations derived in Section 3.2.1. A charge collection length $\left(d_{e}\right.$ and $\left.d_{h}\right)$ of $500 \mathrm{~nm}$ is used. This value was used in order to show the variation of results from model. The plots of charge collection $\left(\mathrm{Q}_{\mathrm{Q}} \mathrm{Q}_{\mathrm{o}}\right)$ as a function of absorption coefficient $\left(\mathrm{nm}^{-1}\right)$ are shown for different thicknesses $(100,200$, 300, 500 and $1000 \mathrm{~nm}$ ) of P3HT solar cells. 

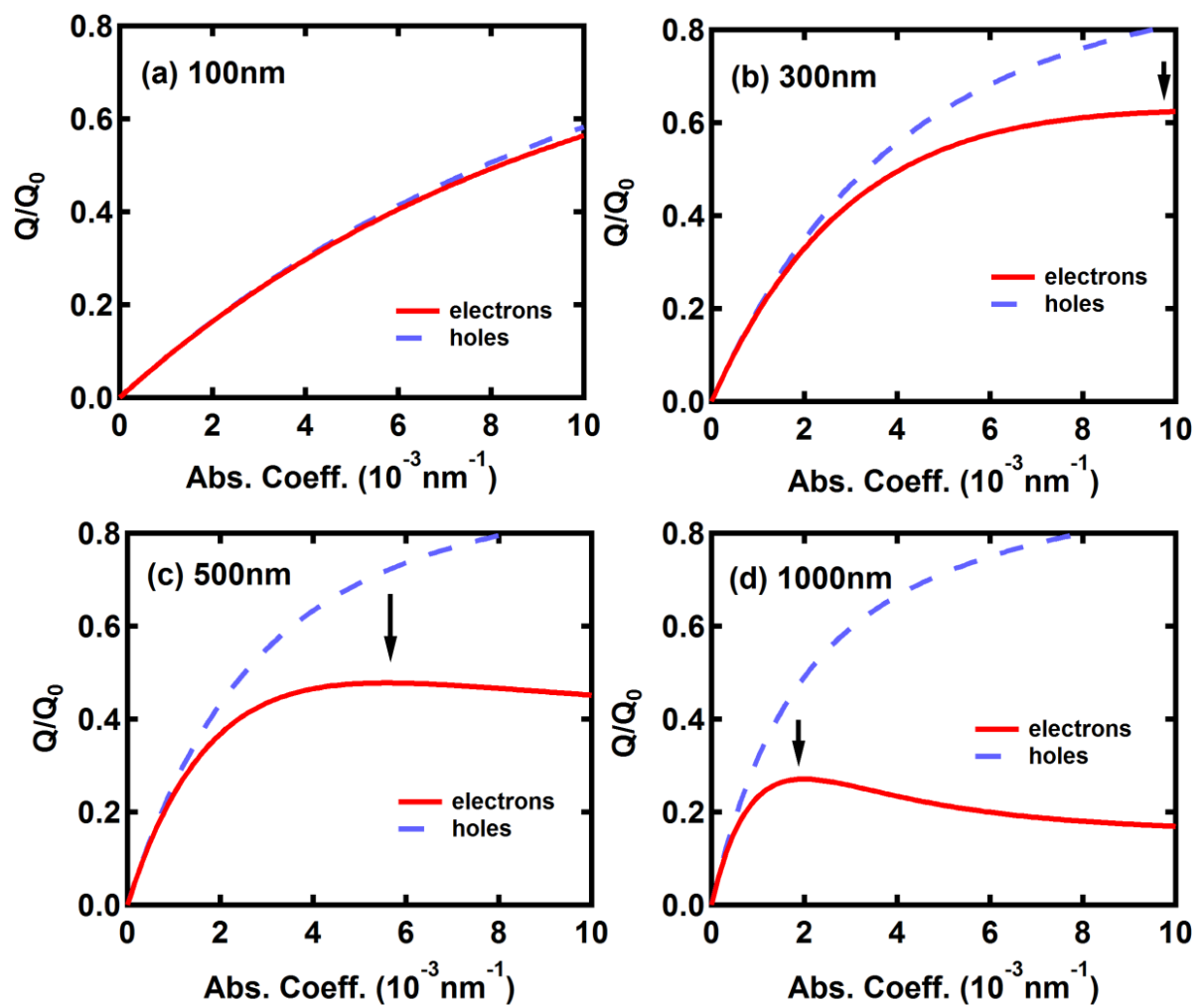

Figure 3.8: (a). The fraction of extracted charge as a function of the absorption coefficient for active layer thicknesses of (a) $100 \mathrm{~nm}$, (b) $300 \mathrm{~nm}$, (c) $500 \mathrm{~nm}$ and (d) $1000 \mathrm{~nm}$.

There are two important features observed in these plots. The first feature is that, the charge collection for both electrons and holes maximized at an absorption coefficient value which is not the maximum absorption value. The second feature is the, decrease of electron collection charge as a function of absorption coefficient. For $100 \mathrm{~nm}$ thin device, the electron and hole collection charges almost lie on each other but when the thickness is increased, the electron collection charge decreases tremendously. In order to get the total photocurrent in these devices it is necessary to have both electron and hole collected charge at the electrodes. Since the electron charge collection decreases with increasing thickness according to the above graph, the smaller value of the carrier type, that is electrons make the necessary contribution for the total photocurrent. In other words, the 
smaller of $\mathrm{Q}_{\mathrm{e}}$ and $\mathrm{Q}_{\mathrm{h}}$ is proportional to the total photocurrent of the device. This important carrier type is called 'collection charge', which is denoted by $\mathrm{Q}_{\mathrm{c}}$. This charge collection $\mathrm{Q}_{\mathrm{c}}$ is plotted as a function of absorption coefficient for P3HT solar cells for thicknesses from 100 to $1000 \mathrm{~nm}$ which is shown in next section.

\subsubsection{Charge collection dependence on absorption coefficient and thickness}

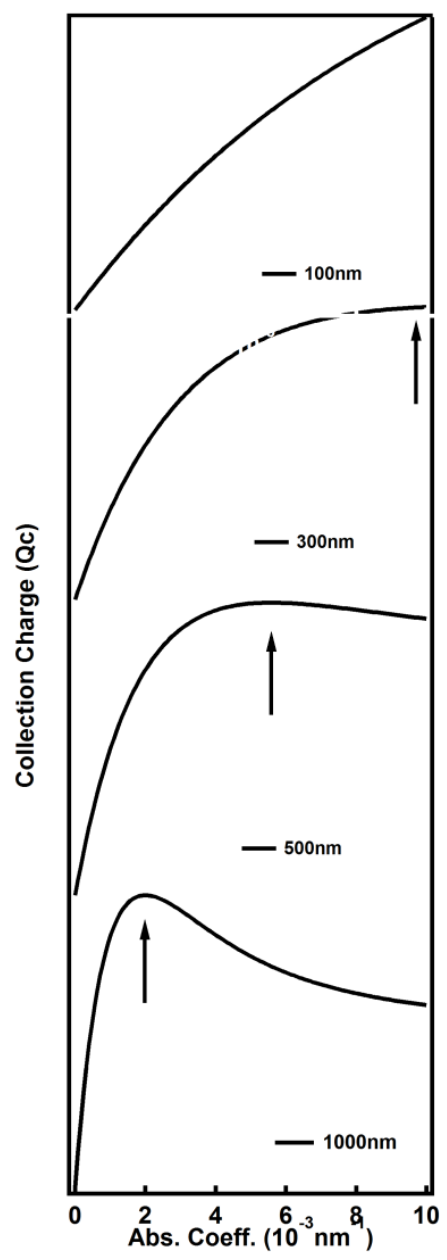

Figure 3.9: The charge collection $\mathrm{Q}_{\mathrm{c}}$ plotted as a function of absorption coefficient of P3HT samples for varying device thickness. 
The charge collection $\mathrm{Q}_{\mathrm{c}}$, plotted as a function of absorption coefficient is shown in above graph for thicknesses from $100 \mathrm{~nm}$ to $1000 \mathrm{~nm}$. Main interesting feature in these curves is the peak of the charge collection shifts to lower end of the absorption profile with increasing thickness. Also it is clearly observed that this peak of the charge collection decreases with increasing thickness. For an example, for thickness of $100 \mathrm{~nm}$, the peak of the charge collection lies at $19.6 \times 10^{-3} \mathrm{~nm}^{-1}$ while this value reduces to $2.0 \mathrm{x}$ $10^{-3} \mathrm{~nm}^{-1}$ for the device thickness of $1000 \mathrm{~nm}$ when $d_{e}=d_{h}=500 \mathrm{~nm}$.

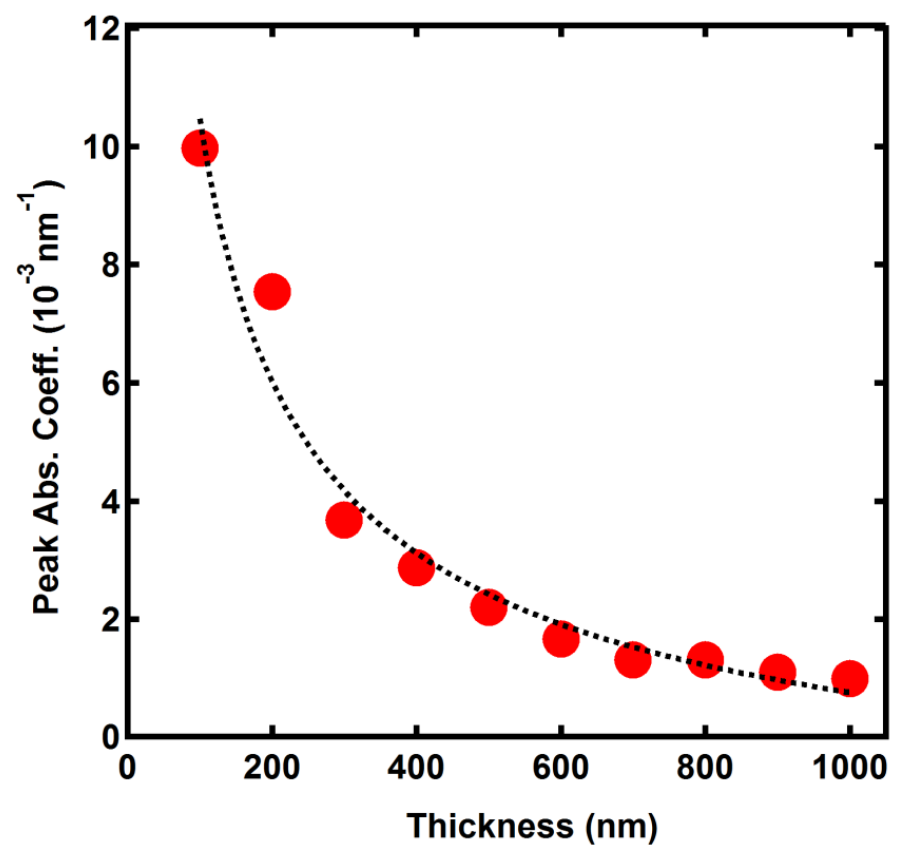

Figure 3.10: The absorption coefficient value corresponds to peak positions of above P3HT samples plotted as a function of device thickness.

Next the absorption coefficient values correspond to the peak positions of the P3HT devices are plotted as a function of device thickness. It is clear evident that increasing thickness of the device, reduces carrier absorption and hence the total photocurrent. For the device of $100 \mathrm{~nm}$, the absorption of the peak is maximized that matches with 
absorption profile of P3HT samples. With increasing thickness, the values decreases that fits to a power curve. This plot of peak absorption coefficient plotted as a function of thickness can be used to determine various properties of model. For example, It can be used to identify the ratio of $d_{e} / d_{h}$, the surface recombination distance from the contacts and mobilities of the materials.

\subsubsection{Charge collections spectra for P3HT and MDMO-PPV samples}

Following is the collection charge $\mathrm{Q}_{\mathrm{c}}$ plotted separately as a function of wavelength for different thicknesses of P3HT and MDMO-PPV devices. These plots are normalized with respect to the maximum charge collection to compare the variation of peak position. The important feature here is that the collection charge shifts to longer wavelengths with respect to the device thickness. For thin devices $(100 \mathrm{~nm})$, the peak of charge collection (electron) matches with main absorption but with increasing thickness, the electron collection charge shifts to longer wavelength region of the spectrum. This observation has not seen with holes collection charge. 

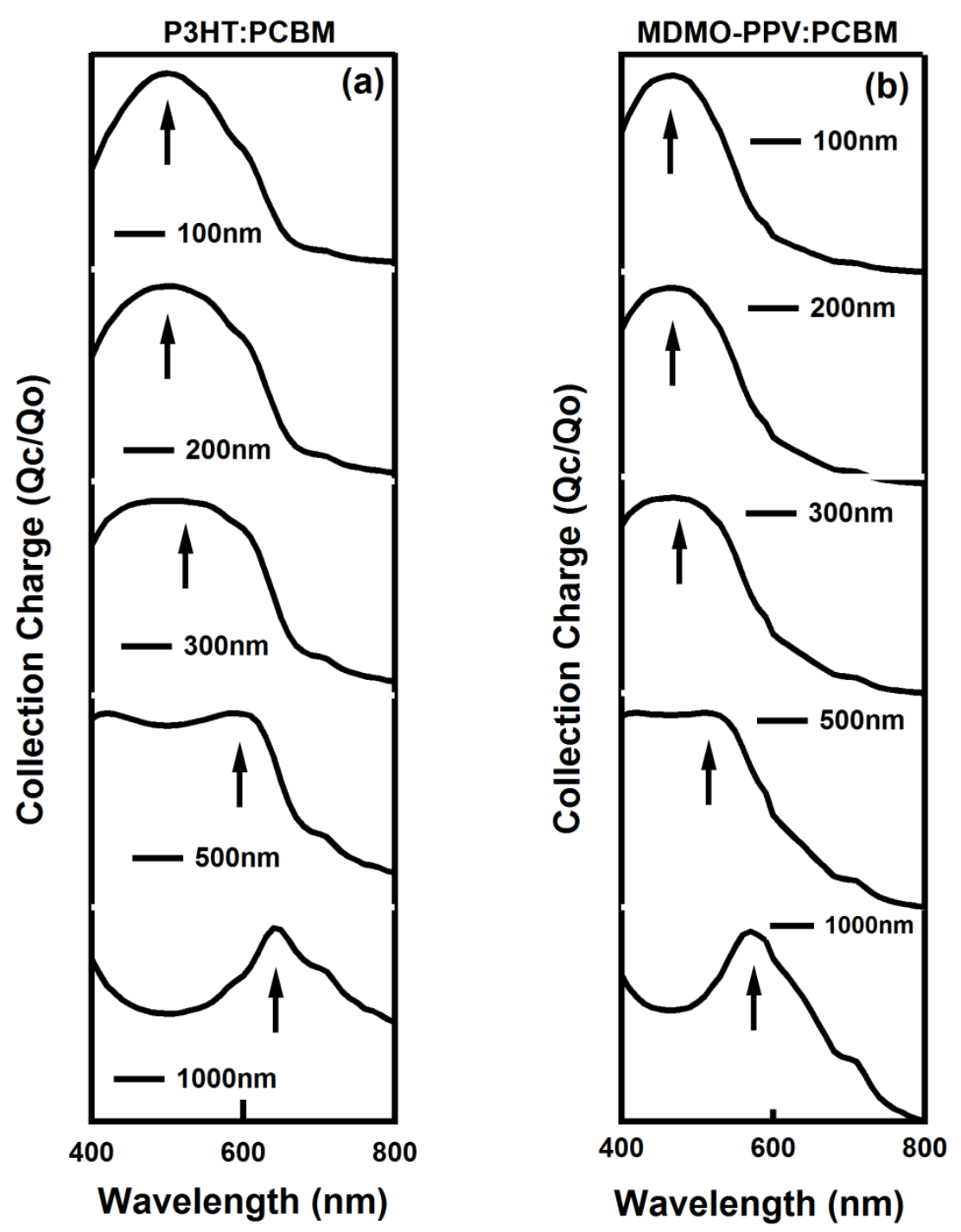

Figure 3.11: Fraction of charge collected $\left(\mathrm{Q}_{\mathrm{c}}\right)$ spectra for five different active layers thickness of (a). P3HT: PCBM (b). MDMO-PPV: PCBM solar cells for the hole and electron collection lengths of $500 \mathrm{~nm}$.

A similar calculation is done using the absorption profile of the MDMO-PPV samples and the plot is shown in the same figure (b) above. Again the collection charge shifts to longer wavelength region with increasing thickness. The data is normalized with respect to the maximum collection charge for the comparison. 


\subsubsection{Absorption coefficient dependence on thickness for different charge collection lengths}

This section shows the charge collection dependence on thickness for varying charge collection distances. It is assumed that the charge collection lengths $\left(\mathrm{d}_{\mathrm{e}}, \mathrm{d}_{\mathrm{h}}\right)$ are proportional to the mobilities of the samples and hence the charge collection distance dependence on mobilities are calculated using this model. The log-log graphs show the electron charge fraction for peak absorbance as a function of device thickness.

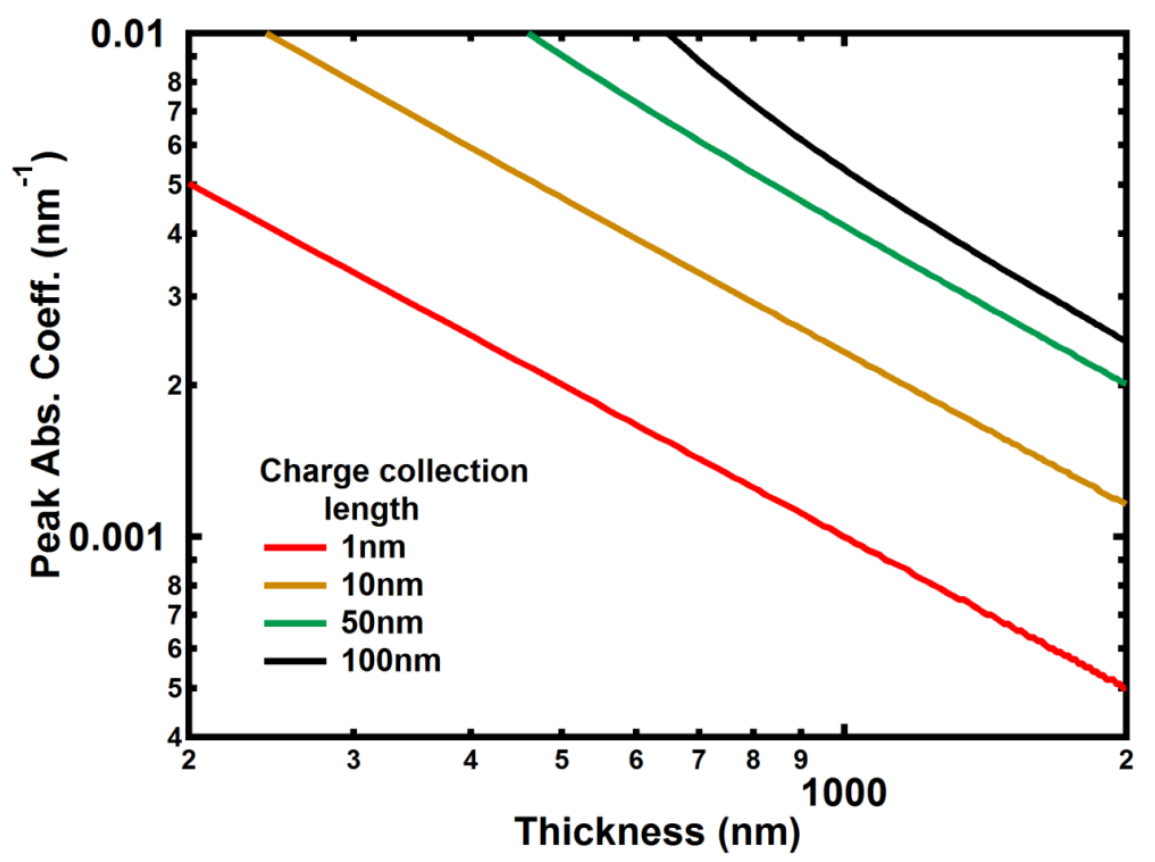

Figure 3.12: The absorption coefficient measured at the extracted charge peak for various collection lengths for electrons of P3HT:PCBM solar cells for surface recombination length of $0 \mathrm{~nm}$.

In this case the holes collection charge does not change with collection length. However peak absorbance in these devices decreases with decreasing collection length. Since the collection length is linearly proportional to the mobility of the device, the peak 
absorbance for the maximum electron collection decreases with electron mobility of the device. This dependence does not true for holes collection charge since there is no dependence shown with holes collection charge to the mobility of the device.

\subsubsection{Peak absorption coefficient dependence on thickness for different}

\section{mobilities}

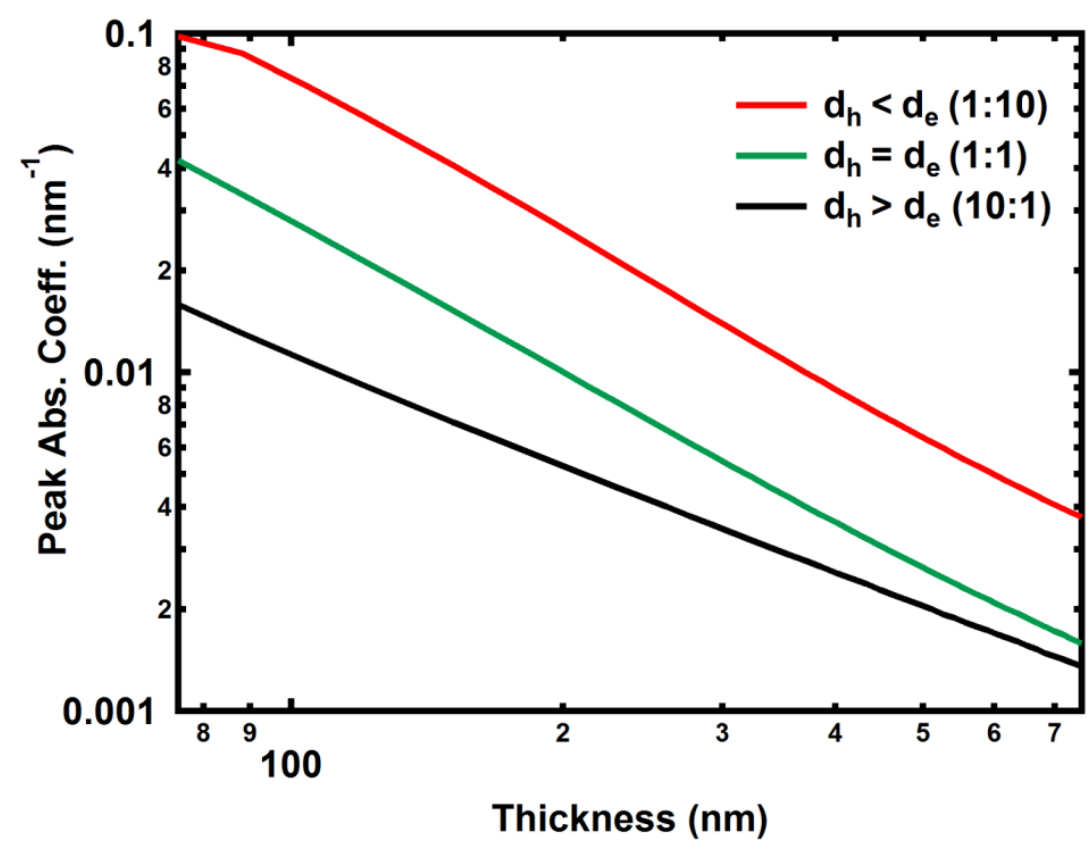

Figure 3.13: The peak absorption coefficient measured at the extracted charge peak for three cases of $d_{e}>d_{h}, d_{e}<d_{h}$ and $d_{e}=d_{h}$ for P3HT/PCBM solar cells.

Figure 3.13 shows $\log -\log$ plot of the peak absorption coefficient as a function of thickness for the cases of $d_{e}>d_{h}, d_{e}<d_{h}$ and $d_{e}=d_{h}$. The surface recombination, $d_{s}$ for this calculation was taken as zero. According to the graph, when $d_{e}>d_{h}$, the absorption is maximized for thinner samples but decreases for the two cases of $d_{e}<d_{h}$ and $d_{e}=d_{h}$. The lowest peak absorption occurs for $\mathrm{d}_{\mathrm{e}}<\mathrm{d}_{\mathrm{h}}$. 


\subsubsection{Derivation of the model for back illumination}

When illumination is done back side instead of front side as shown in the Figure 3.14, the electron charges should travel a distance of $\mathrm{d}-\mathrm{x}$ and holes travel a distance of $\mathrm{x}$.

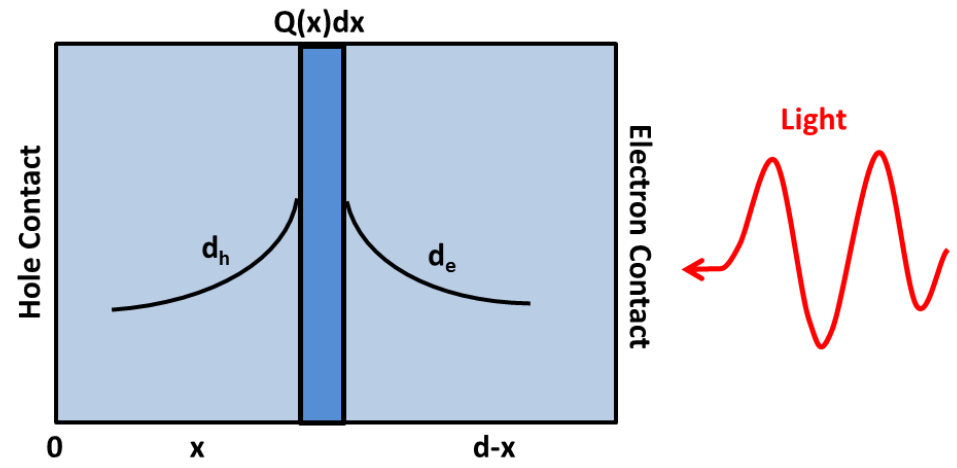

Figure 3.14: Generation of population of charge $\mathrm{Q}(\mathrm{X})$ at a distance $\mathrm{dx}$ from the front contact decays exponentially due to recombination. Here the light illuminates from back contact.

Hence the derivation of fraction of electrons collected by the back electrode when the illumination is done from the back is as follows. The charge collected from the back electrode is given by

$$
Q(x)=Q_{0} \alpha e^{-\alpha(d-x)}
$$

These charges travel distance of $d-x$ with a collection length of $d_{e}$. Hence the charge extracted from the aluminum (Al) electrode will be given by 


$$
d Q_{e}(x)=Q_{0} \alpha e^{-\alpha(d-x)} e^{-(d-x) / d_{e}} d x
$$

Distance $d_{s}$ is introduced as the recombination distance from the surface which all carriers are recombined due to surface interface states.

$$
\begin{aligned}
\int_{0}^{Q_{e}} d Q_{e}(x) & =\int_{0}^{d-d_{s}} Q_{0} \propto e^{-d\left(\alpha+\frac{1}{d_{e}}\right)} \cdot e^{x\left(\alpha+\frac{1}{d_{e}}\right)} d x \\
\frac{Q_{e}}{Q_{0}} & =\frac{\alpha}{\left(\alpha+\frac{1}{d_{e}}\right)} e^{-d\left(\alpha+\frac{1}{d_{e}}\right)} \cdot\left(e^{\left(d-d_{s}\right) \cdot\left(\alpha+\frac{1}{d_{e}}\right)}-1\right) \\
\frac{Q_{e}}{Q_{0}} & =\frac{\alpha}{\left(\alpha+\frac{1}{d_{e}}\right)} \cdot\left[e^{-d_{s}\left(\alpha+\frac{1}{d_{e}}\right)}-e^{-d\left(\alpha+\frac{1}{d_{e}}\right)}\right]
\end{aligned}
$$

When the surface recombination distance $d_{s}=0$, above Equation 3.27 becomes

$$
\frac{Q_{e}}{Q_{0}}=\frac{\propto}{\left(\propto+\frac{1}{d_{e}}\right)} \cdot\left[1-e^{-d\left(\alpha+\frac{1}{d_{e}}\right)}\right]
$$

Equation 3.28 is same as Equation 3.20 for the holes extracted charge when illumination is from ITO side. Similarly for holes extraction one would write

$$
\begin{gathered}
d Q_{h}(x)=Q_{0} \alpha e^{-\alpha(d-x)} e^{-x / d_{h}} d x \\
\int_{0}^{Q_{h}} d Q_{h}(x)=\int_{0}^{d-d_{s}} Q_{0} \propto e^{-d(\alpha)} \cdot e^{x\left(\alpha-\frac{1}{d_{h}}\right)} d x \\
\frac{Q_{h}}{Q_{0}}=\frac{\alpha e^{-\alpha d}}{\left(\alpha-\frac{1}{d_{h}}\right)} \cdot\left(e^{\left(d-d_{s}\right) \cdot\left(\alpha-\frac{1}{d_{h}}\right)}-1\right)
\end{gathered}
$$




$$
\begin{gathered}
\text { Multiply by }\left[( - ) \left(e^{\left.\frac{-d}{d_{h}}\right)} \cdot(-)\left(e^{\left.\frac{d}{d_{h}}\right)}\right)\right.\right. \\
\frac{Q_{h}}{Q_{0}}=\frac{\propto e^{-\left(\frac{d}{d_{h}}\right)}}{\left(\frac{1}{d_{h}}-\propto\right)} \cdot\left(e^{d\left(\frac{1}{d_{h}}-\propto\right)}-e^{\left(d-d_{s}\right)\left(\propto-\frac{1}{d_{h}}\right)} \cdot e^{-d\left(\propto-\frac{1}{d_{h}}\right)}\right) \\
\frac{Q_{h}}{Q_{0}}=\frac{\propto e^{-\left(\frac{d}{d_{h}}\right)}}{\left(\frac{1}{d_{h}}-\propto\right)} \cdot\left[e^{d\left(\frac{1}{d_{h}}-\propto\right)}-e^{d_{s}\left(\frac{1}{d_{h}}-\propto\right)}\right] \\
\text { For } \mathrm{d}_{\mathrm{s}}=0, \frac{Q_{h}}{Q_{0}}=\frac{\propto e^{-\left(\frac{d}{d_{h}}\right)}}{\left(\frac{1}{d_{h}}-\propto\right)} \cdot\left[e^{d\left(\frac{1}{d_{h}}-\propto\right)}-1\right]
\end{gathered}
$$

Equation 3.34 is same as Equation 3.22 for the electron extracted charge when illumination is done from ITO side. Above electron and holes charge fraction give a good relationship between the photogenerated charges extracted with respect to the active layer thickness. This also has the $\mathrm{d}_{\mathrm{s}}$ term which allows obtaining approximate surface recombination distance for the organic BHJs. Results of the model calculations used to describe thickness dependence of organic BHJs will be discussed in next chapter.

\subsection{Front/Back Illumination effect on charge collection}

This model can be used to see the charge collection effect for different interfaces. One of the interesting ideas is to see the difference just by changing the illumination side. This demonstrates the effect on electron collection with device thickness by front (ITO contact) and back (Al contact) side illumination. Following graph shows the absorption coefficient corresponds to peak of the collection charge as a function of device thickness for the two cases. To do this calculation, the surface recombination distance of $10 \mathrm{~nm}$ is 
used. The electron and holes mobility values of $\mu_{n}=2 \times 10^{-4} \frac{\mathrm{cm}^{2}}{V s}$ and $\mu_{h}=1.4 \times$ $10^{-4} \frac{\mathrm{cm}^{2}}{V s}$ respectively are used for collection length components since they are proportional to each other. When the illumination is done from the back side, the electron and holes mobilities are switched since the electron collection fraction for back side illumination becomes holes for front illumination.
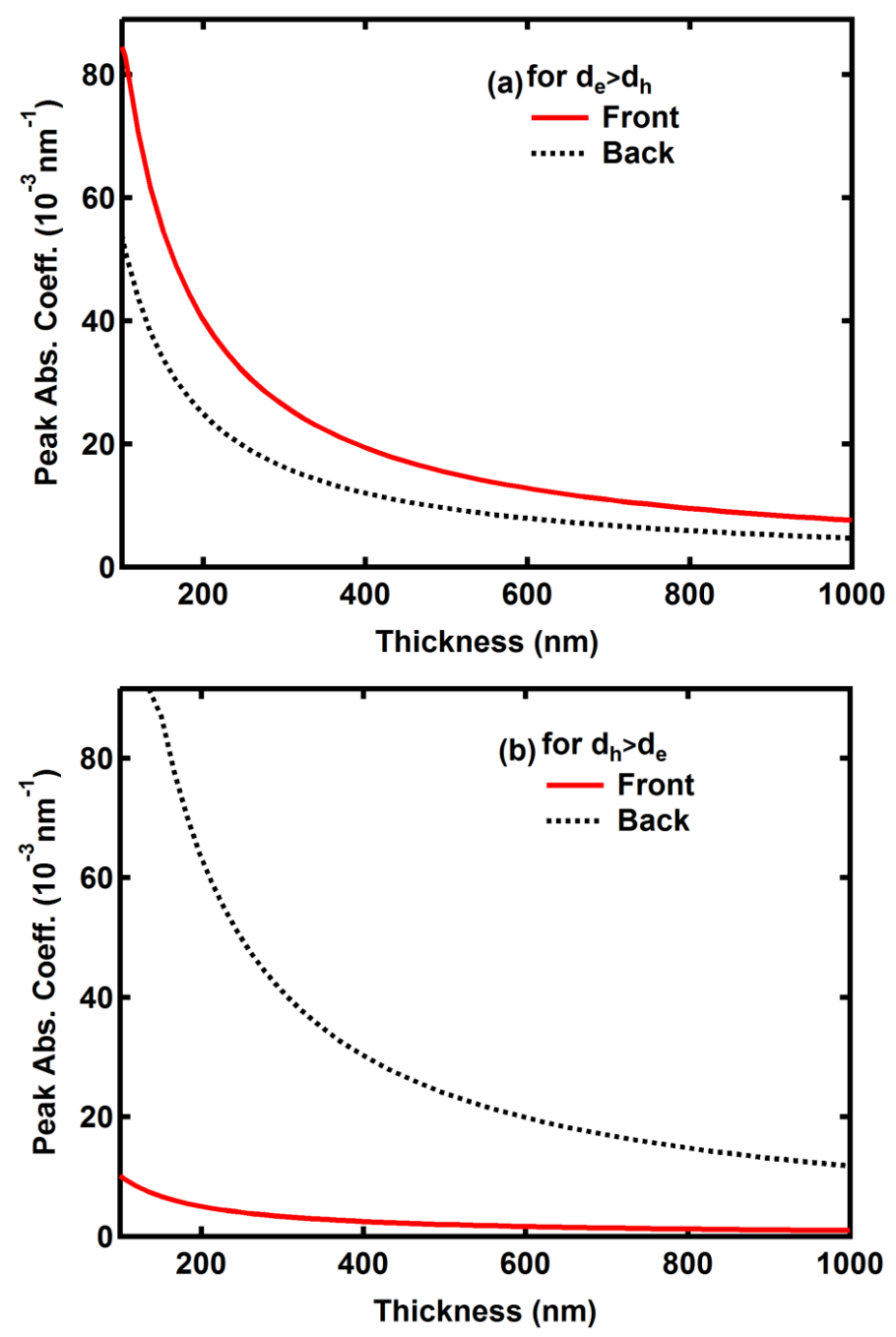

Figure 3.15: The absorption coefficient measured at the extracted charge peak for the case of front and back illumination in P3HT/PCBM solar cells for (a) $d_{e}>d_{h}$ and (b) $d_{h}>d_{e}$. 
Here we can observe that for front illumination, there is a high exciton generation occurs hence a larger absorption effect takes place compared to that of back illumination. In general both show a reduction of charge collection at maximum absorbance with increasing thickness.

\subsection{Charge collection dependence on surface recombination in P3HT samples}

Charge collection fraction dependence on the surface recombination is discussed in this section. Distance $d_{s}$ in used as surface recombination distance in which all the carriers generated within this distance recombined or trapped in metal-semiconductor interface states. Hence to calculate the total holes collected from front electrode at front illumination is given by equation 3.17 ,

$$
\begin{aligned}
Q_{h}(x) & =\int_{0}^{Q_{h}} d Q_{h}(x)=\int_{d_{s}}^{d} Q_{0} \alpha e^{-\alpha x} e^{-x / d_{h}} d x \\
\frac{Q_{h}}{Q_{0}} & =\frac{\propto}{\left(\propto+\frac{1}{d_{h}}\right)}\left[e^{-d_{s}\left(\propto+\frac{1}{d_{h}}\right)}-e^{-d\left(\propto+\frac{1}{d_{h}}\right)}\right]
\end{aligned}
$$

Similarly for collected electrons from the back contact with the surface recombination term is given by

$$
\begin{aligned}
Q_{e}(x)=\int_{0}^{Q_{e}} d Q_{e}(x) & =\int_{d}^{d_{s}} Q_{0} \alpha e^{-\alpha x} e^{-(d-x) / d_{e}} d x \\
\frac{Q_{e}}{Q_{0}} & =\propto e^{\frac{-d}{d_{e}}}\left[\frac{e^{\left(d_{s}-d\right)\left(\frac{1}{d_{e}}-\alpha\right)}}{\left(\frac{1}{d_{e}}-\propto\right)}\right]
\end{aligned}
$$




$$
\frac{Q_{e}}{Q_{0}}=\frac{\propto e^{-\left(d / d_{e}\right)}}{\left(\frac{1}{d_{e}}-\propto\right)}\left[e^{d\left(\frac{1}{d_{e}}-\propto\right)}-e^{d_{s}\left(\frac{1}{d_{e}}-\propto\right)}\right]
$$

For this calculation, the charge collection lengths $\left(\mathrm{d}_{\mathrm{e}}\right.$ and $\left.\mathrm{d}_{\mathrm{h}}\right)$ are kept constant at $500 \mathrm{~nm}$ and surface recombination length $\left(\mathrm{d}_{\mathrm{s}}\right)$ has been changed with respect to the active layer thickness (d).

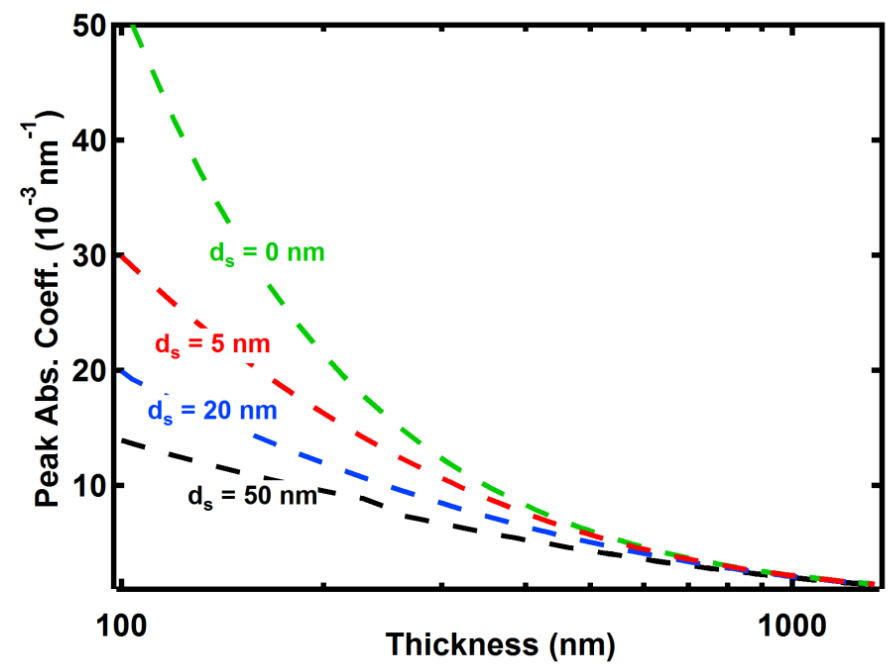

Figure 3.16: The absorption coefficient measured at the extracted charge peak for different recombination distances in P3HT: PCBM solar cells.

The above graph shows the peak absorption coefficient value for the maximum charge collection fraction as a function of device thickness for surface recombination lengths (ds) of 0, 5, 20 and $50 \mathrm{~nm}$. Charge extraction maximizes at maximum absorbance when the device does not have any surface recombination. Furthermore, model calculation shown in the above graph, when surface recombination distance increases from 0 to $50 \mathrm{~nm}$, the absorption and the generation of charge carriers decreases producing less photocurrent. Also the absorption is highest at thinner samples and shows a decrease with thicker devices. 


\section{CHAPTER IV: EXPERIMENTAL PHOTOCURRENT RESULTS}

\subsection{Introduction}

The first part of this chapter is dedicated to photocurrent measurements that make up the main results of this work. The photocurrent dependence on different active layer thicknesses of P3HT: PCBM and MDMO-PPV: PCBM polymer solar cells are discussed. In this part, the theoretical values for absorption coefficient variation with thickness are compared with experimental data. The photocurrent variation due to different interfacial layers will be discussed. Different recombination distances were used to show the dependence of absorption coefficient on thickness for P3HT samples. Experimental data is plotted along with model in order to show the comparison. Thin layers of PEDOT-PSS and P3HT are used as the interfacial layers to see the effect of photocurrent dependence on thickness. Then the main results of red shift of the photocurrent will be discussed. Model fittings used in this section help to find the charge collection lengths of these types of solar cells. Finally the effect on photocurrent for front and back illuminations of the solar cells is also discussed.

The photocurrent of organic solar cells describes the photo-induced exciton generation, exciton dissociation and charge transport mechanism of BHJs. In basic definition by analyzing the optical absorption profile of a material, the profile of photocurrent can be suggested. Normally the photocurrent must be matched with 
absorption profile that tells the maximum photo-induced charge generation. On the other hand, photoluminescence of a material describes the minimum energy transition of a semiconducting energy band. That is energy corresponds to maximum Photoluminescence equals to the minimum energy transition between the HOMO and LUMO levels of organic structure. The absorption and photoluminescence profile of MDMO-PPV: PCBM and P3HT: PCBM BHJ solar cells are shown in the following figure.
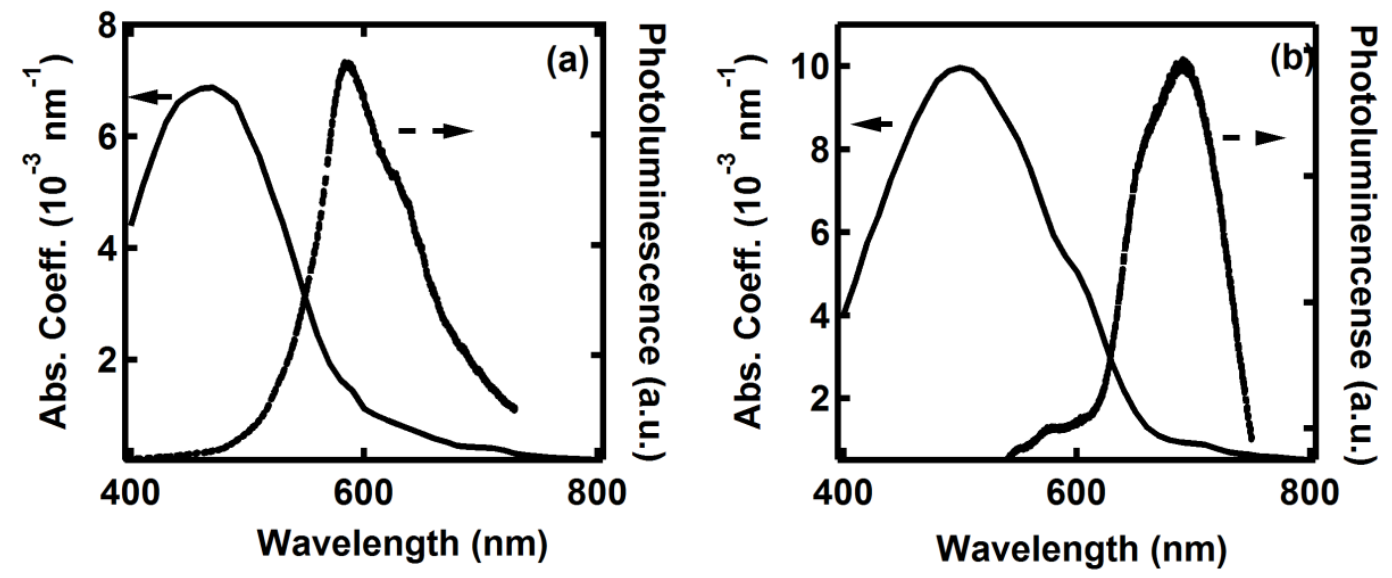

Figure 4.1: Absorption and Photoluminescence spectra of (a) MDMO-PPV: PCBM and

(b) P3HT: PCBM solar cells.

MDMO-PPV: PCBM devices show an absorption maximum at 470nm and P3HT: PCBM shows a maximum at 510nm. This stoke shift behavior between the absorbance and luminescence has been observed by Street et al[23]. When light absorbs, the electrons in HOMO level jumps to energy states with energy higher than the LUMO level. But within a fraction of picoseconds, they relax to lower states and finally to band edge before radiatively recombining with a hole in HOMO level. This process occurred by a thermalization of absorbed carriers[64]. Photoluminescence spectra for these organic 
structures are obtained by $325 \mathrm{~nm}$ excitation from a He-Cd laser using a Renishaw RL633 spectrometer. Then the photocurrent spectra are measured for MDMO-PPV: PCBM organic bulk heterojunction solar cells for different thicknesses ranging from 0 to $2 \mu \mathrm{m}$. Different thicknesses were obtained by changing the spin speeds of the coating layer. The thicknesses were measured using Veeco 8 Dektek profilometer. Except spin speeds, all other variables for these samples kept constant so that the variation of thickness on the photocurrent can be easily observed.

\subsection{Effect of interfacial layers on charge collection efficiency}

This section describes how the surface recombination length affects when different interfacial layers are introduced. First the interfacial layer of PEDOT-PSS is used to see how the surface recombination distance of the charge collection fraction changes over the distance. This measurement is compared with a device that has no PEDOT-PSS layer as the interfacial layer. Then a thin layer of P3HT was used as the interfacial layer. A 10nm of P3HT was spin coated on ITO and then annealed at $140^{\circ} \mathrm{C}$ for 10minutes that promotes an adhesion of the layer to ITO. Then the active layer is

deposited on top of the interfacial layer. Annealing step $140^{\circ} \mathrm{C}$ at $10 \mathrm{mins}$ was carried out to make this thin layer less soluble during active layer deposition. 


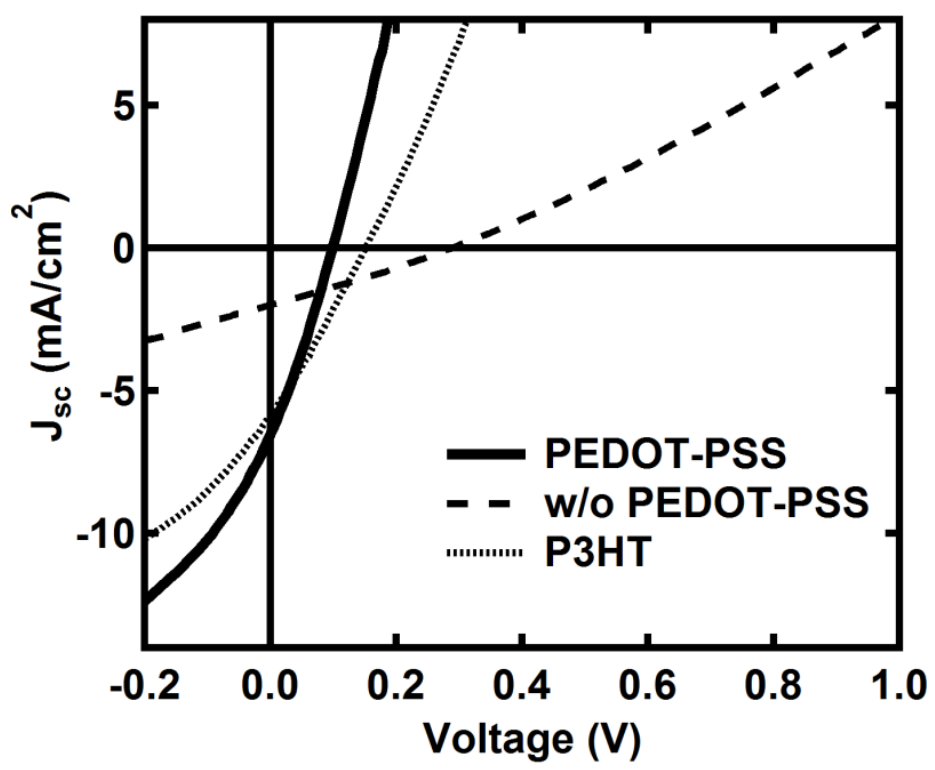

Figure 4.2: The corresponding J-V characteristics of the P3HT: PCBM solar cells with three different interfacial conditions.

The $\mathrm{J}-\mathrm{V}$ characterization of the solar cell devices with different interfacial layers has been done which is shown in the figure above for the cases of with PEDOT-PSS thin layer, without PEDOT-PSS layer and with a thin layer of P3HT. According to the Figure 4.2, the highest short-circuit current gives for the device with PEDOT-PSS and lowest corresponds to the device without PEDOT-PSS.

The following graph (Figure 4.3) shows the comparison of photocurrent with and without PEDOT-PSS layer with respect to thickness. According to the graph 4.3(b), thickest device without PEDOT-PSS clearly show a peak at the long wavelength region while for thinner devices both show peaks at short wavelength region that matches with absorbance shown in top graph. Furthermore according to the graph, devices with PEDOT-PSS show a less red shift in photocurrent with increasing thickness than the devices without PEDOT-PSS. 

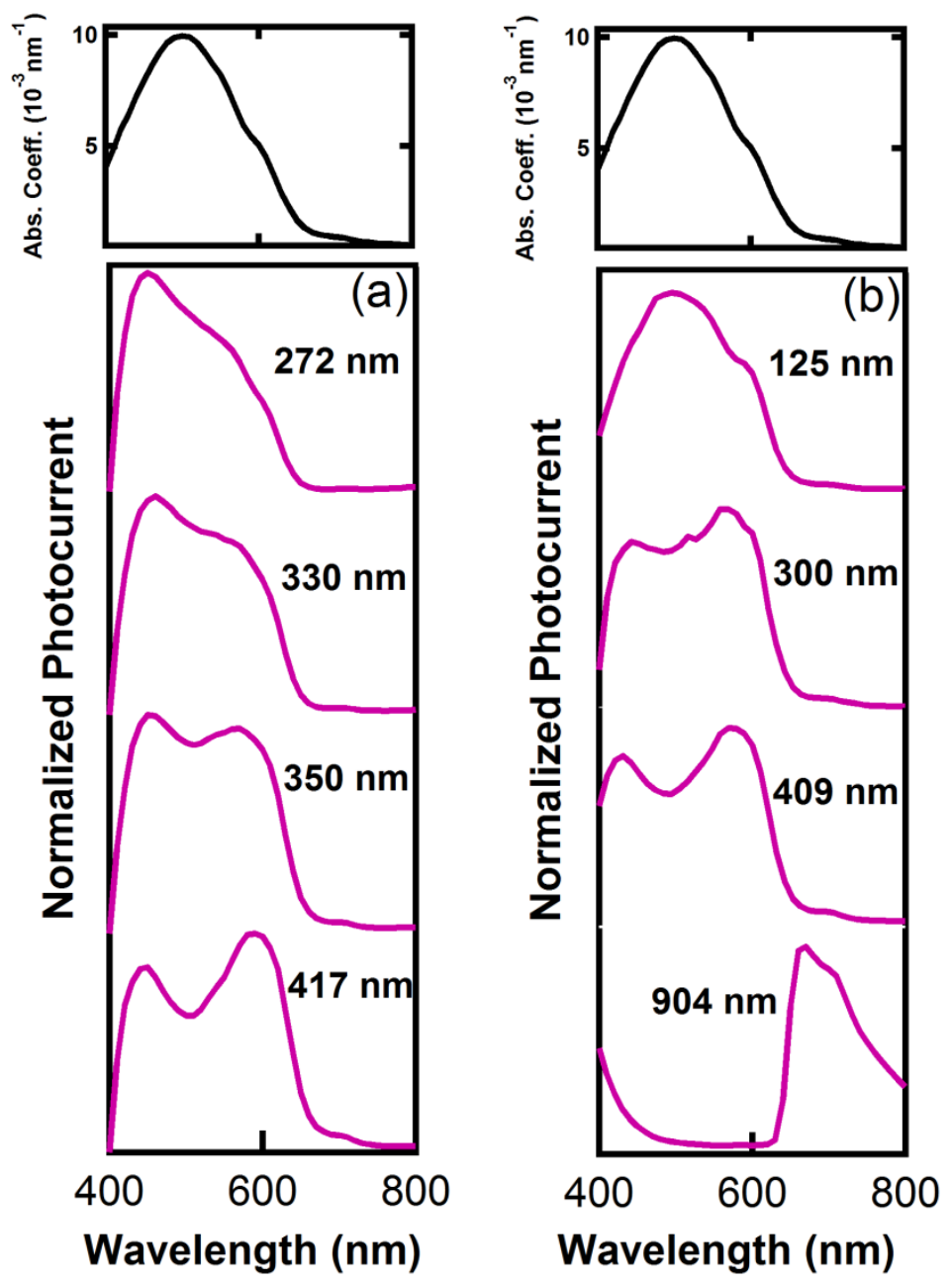

Figure 4.3: Normalized Photocurrent spectra for four different active layer thicknesses of (a). PEDOT-PSS (b). without PEDOT-PSS in P3HT: PCBM solar cells.

To compare this experimental shift with the theoretical model, absorption value corresponds to peak of the photocurrent was taken and plot as a function of device thickness. Then different recombination distances were used to fit into experimental data keeping the electron and holes collection lengths constant. Figure 4.4 shows the absorption coefficient for peak photocurrent as a function of thickness for three cases of PEDOT-PSS, without PEDOT-PSS and with P3HT. 

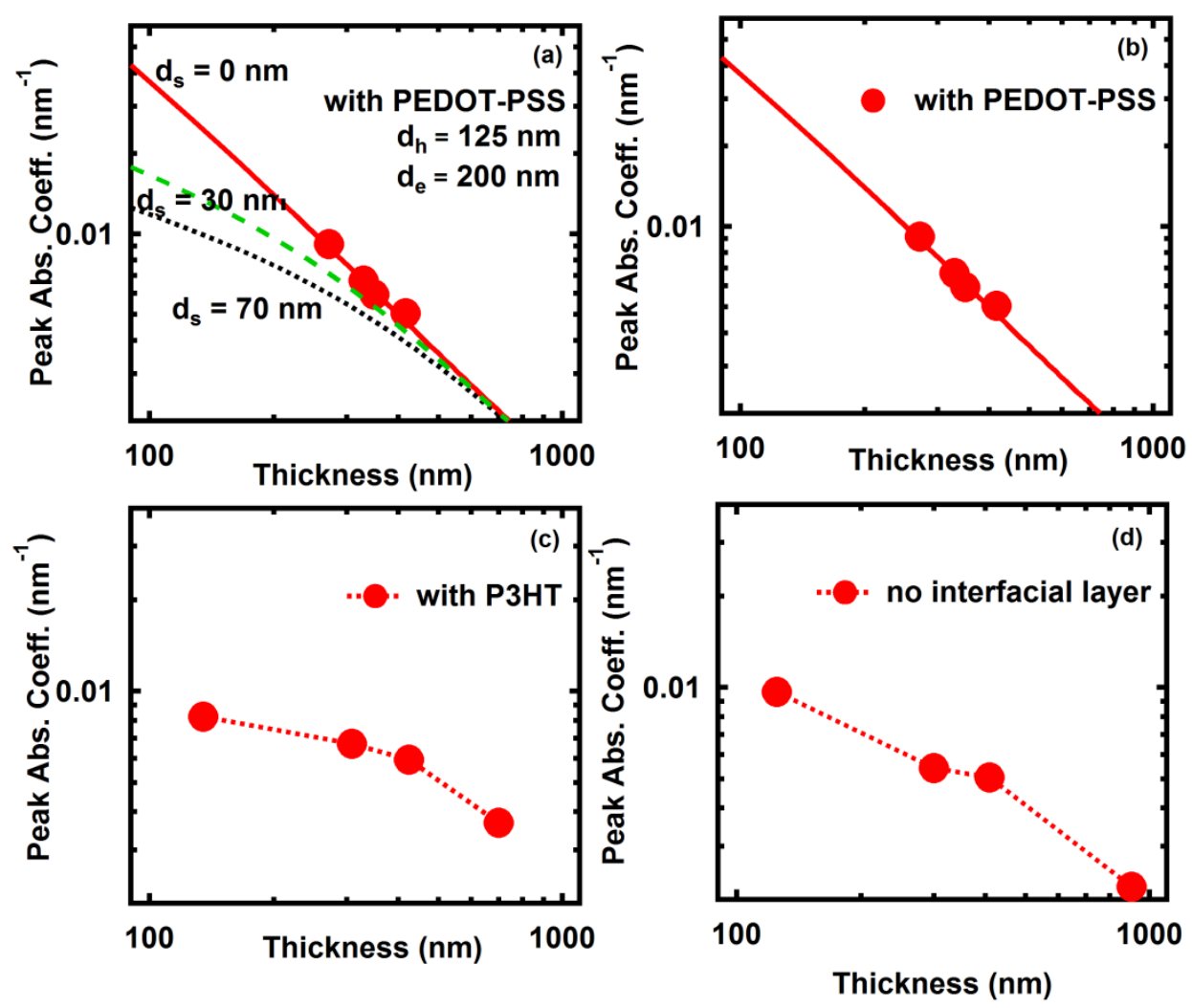

Figure 4.4: The absorption coefficient measured at the photocurrent charge peak for different interfacial layers of P3HT/PCBM solar cells. (a), (b) with PEDOT-PSS, (c) P3HTand (d) without any interfacial layer.

For fitting the model into experimental data, different recombination distances were used. Recombination distance of 0nm was fitted well for PEDOT-PSS. Devices with no interfacial layer and P3HT interfacial layer gave higher recombination lengths that matched with experimental data. Other recombination distances are also shown in the graphs (Figure 4.4 (a)) to show the deviation from the experimental data. According to the model, the higher recombination distance for device without interfacial layer suggests that the interfacial layer is necessary in order to minimize the surface recombination effects in organic polymer solar cells. Also highest performance with minimum 
recombination distance of 0nm gives for the samples with PEDOT-PSS suggests that the importance of PEDOT-PSS layer used as an interfacial layer in organic bulk hetero junctions. Furthermore, this method can be used to study the recombination effect in choosing interfacial layers to optimize organic solar cells.

\subsection{Photocurrent dependence on thickness for P3HT and MDMO-PPV solar cells}

The following figure shows the photocurrent variation with different active layer thicknesses for MDMO-PPV: PCBM organic solar cells for the range of 0 to $2 \mu \mathrm{m}$ (Figure 4.5 (a)). Since the photocurrent values change with sample-to-sample, data normalized to photocurrent peak so that all the peaks can be easily compared.
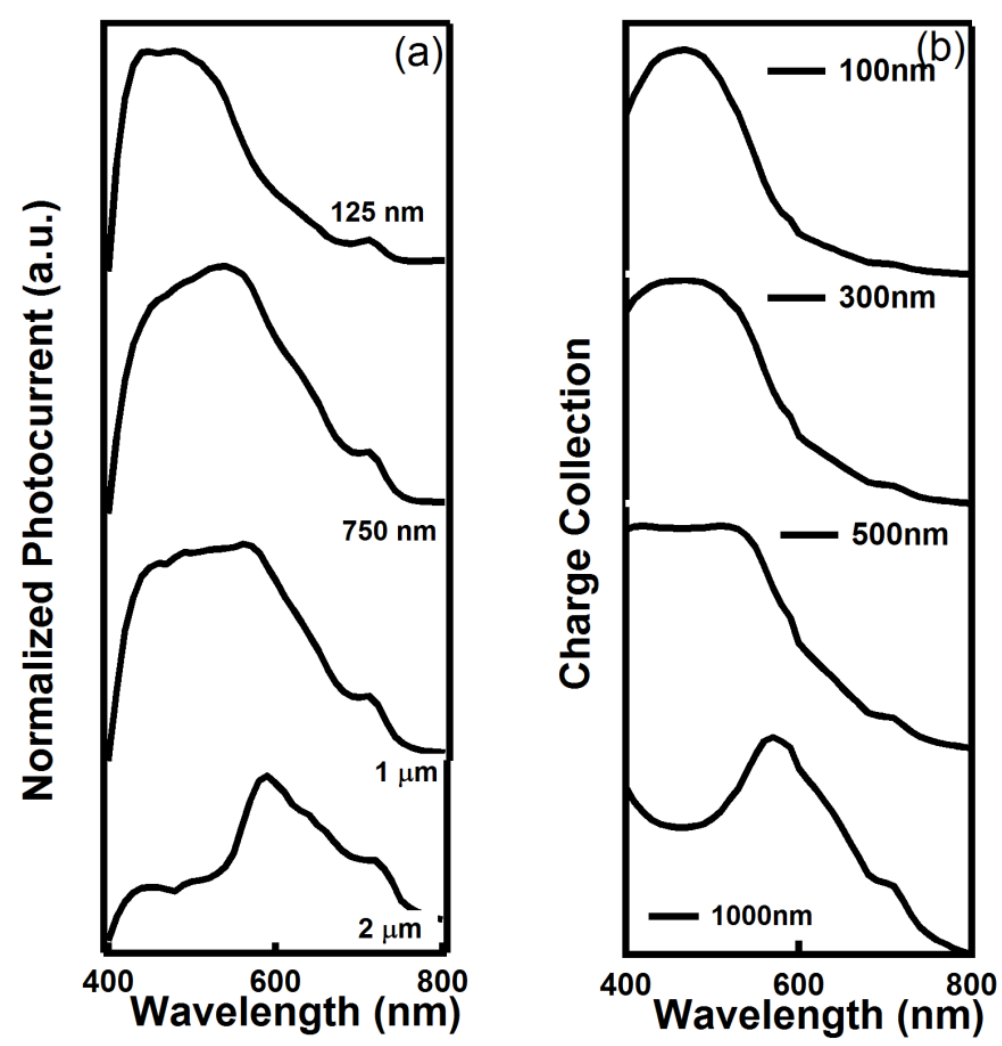

Figure 4.5: The normalized Photocurrent spectra for four different active layer thickness of MDMO-PPV: PCBM solar cells for (a). Experiment (b). Theory $\left(\mathrm{d}_{\mathrm{e}}=500 \mathrm{~nm}\right)$. 
The thinnest MDMO-PPV sample of $125 \mathrm{~nm}$ shows the photocurrent peak that matches with maximum of the absorbance spectrum. The thickest sample of $2 \mu \mathrm{m}$ matches with the luminescence spectrum shown in Figure 4.1. Further increasing of thickness does not show any shift in the photocurrent since it is clearly the maximum shift possible. The peak of the photocurrent clearly shift to longer wavelength region for the samples less than $2 \mu \mathrm{m}$ for MDMO-PPV: PCBM samples[65]. Similar measurement has been done to P3HT: PCBM samples varying the thickness of the active layer. The results are shown in the Figure 4.6.
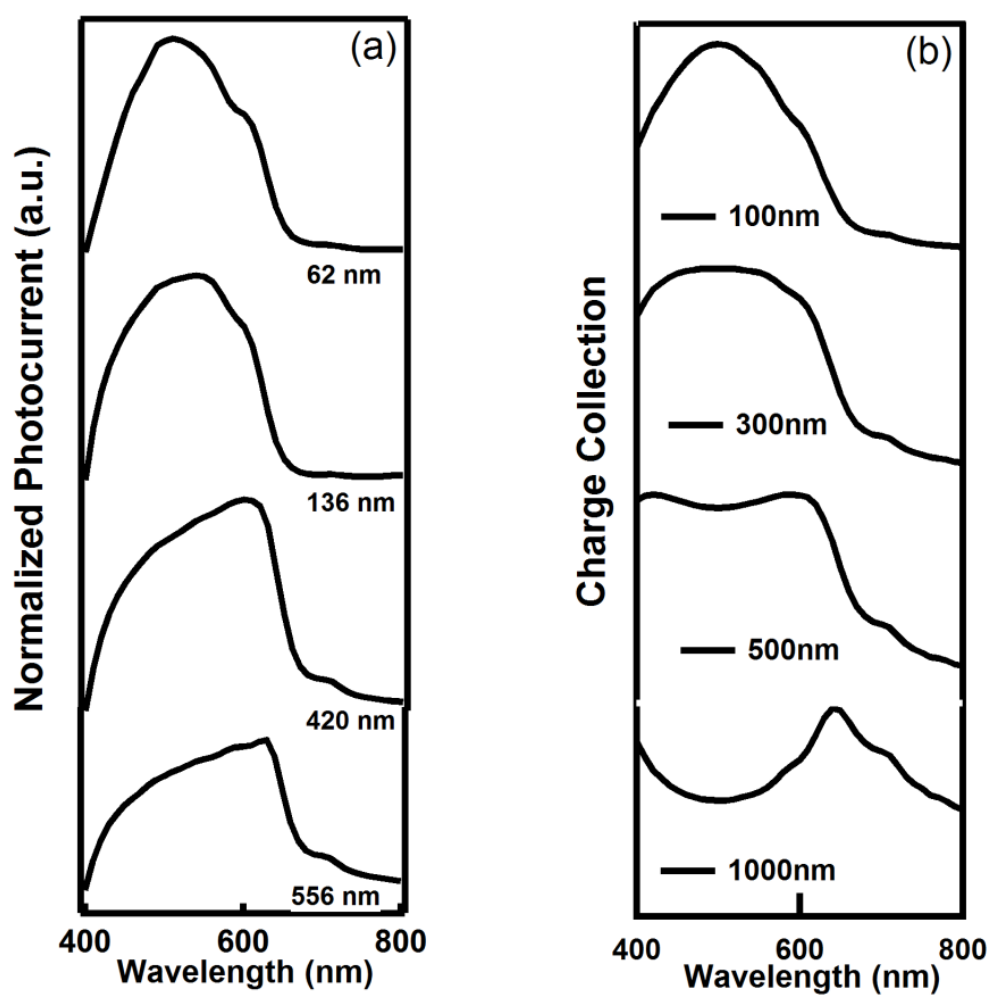

Figure 4.6: The normalized Photocurrent spectra for four different active layer thickness of P3HT: PCBM solar cells for (a). Experiment (b). Theory $\left(d_{e}=500 \mathrm{~nm}\right)$. Again the absorbance and the photoluminescence are compared with graph 4.1 to clearly understand the comparison of the thickness dependence. The peak of the 
photocurrent of thinnest sample matches with the absorbance peak while the thickest sample matches with photoluminescence curve. It is clearly observed that the peak of the photocurrent again red shifts with increasing thickness of P3HT: PCBM structures. A large sample thickness variation is needed to see the shift of the photocurrent peak in MDMO-PPV: PCBM structures because the absorbance in MDMO-PPV structures are lower than that of P3HT. Also another significant feature is that the small shoulder seen at $700 \mathrm{~nm}$ corresponds to PCBM singlet transition remains fixed for all samples in both structures. This can be used as a reference point for the comparison of peak shift in the polymer. Next the absorption coefficient corresponds to the peak of the photocurrent is plotted as a function of thickness of MDMO-PPV: PCBM solar cells which are shown in the figure below. The experimental and theoretical values for different charge collection lengths are also shown in the Figure 4.7 for comparison. 

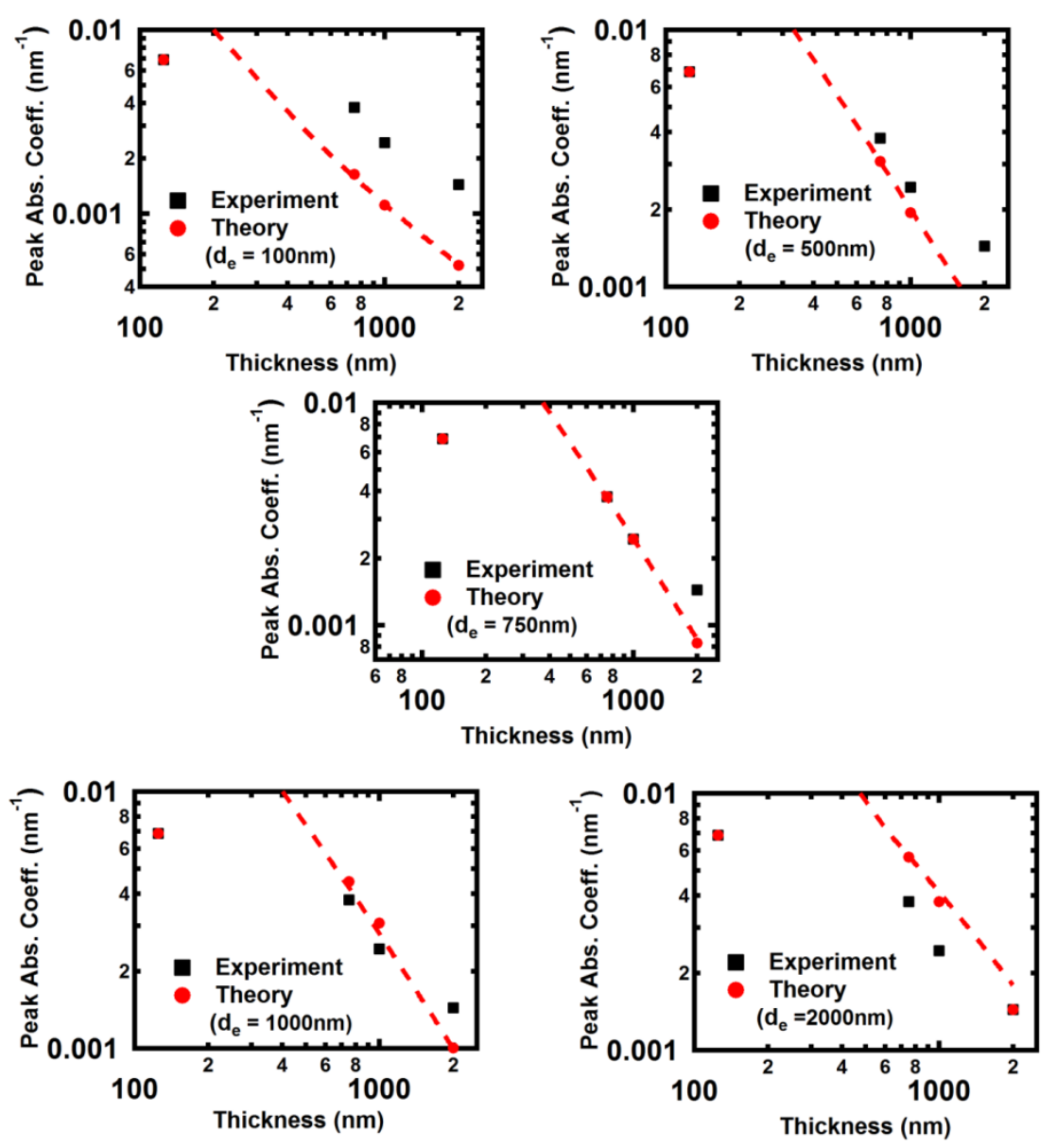

Figure 4.7: The absorption coefficient value corresponds to peak positions of MDMO-

PPV samples plotted as a function of device thickness for experimental and theory (a)

$$
d_{e}=100 \mathrm{~nm},(b) d_{e}=500 \mathrm{~nm}, \text { (c) } d_{e}=750 \mathrm{~nm}, \text { (d) } d_{e}=1000 \mathrm{~nm} \text { and (e) } d_{e}=2000 \mathrm{~nm} \text {. }
$$

According to Figure 4.7, five different charge collection lengths are used to describe how the shift of the peak absorption coefficient changes with experimental data in the log-log plot. Charge collection length of $750 \mathrm{~nm}$ shows the best fit to the experimental data. Deviation from this value gives a poor fit to the experimental data. 


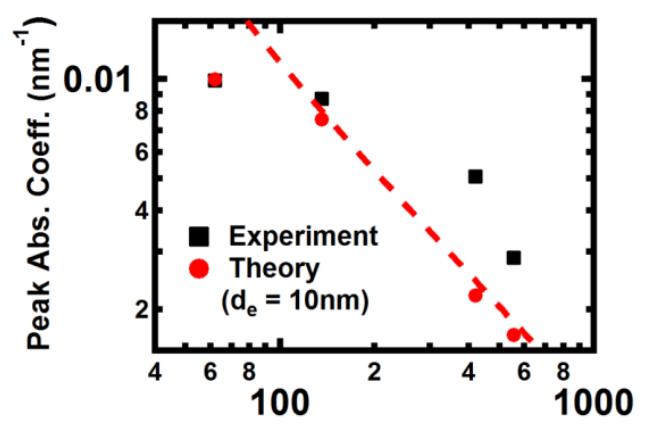

Thickness $(\mathrm{nm})$

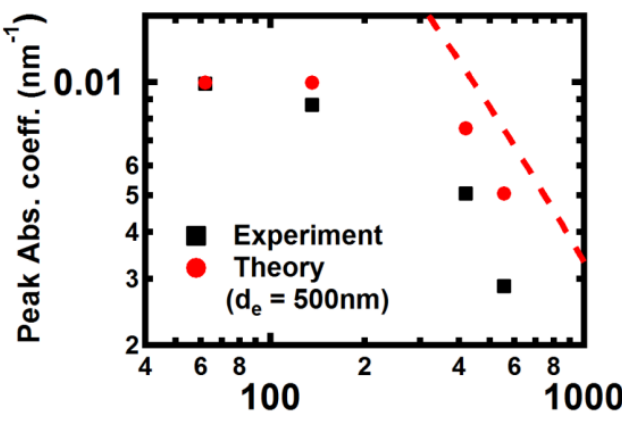

Thickness (nm)

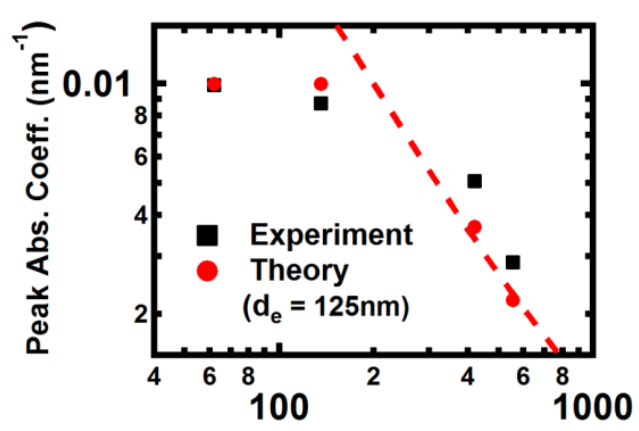

Thickness (nm)

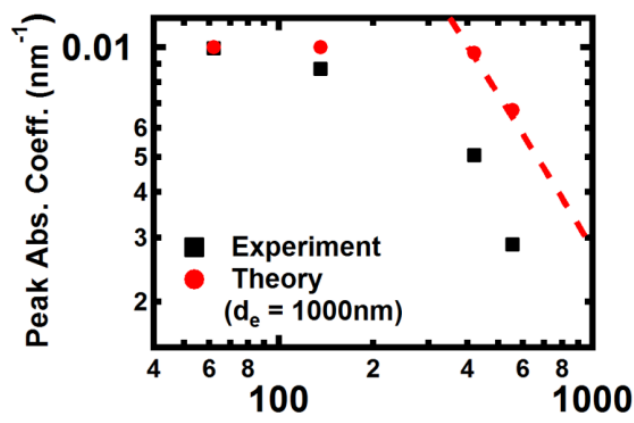

Thickness (nm)

Figure 4.8: The absorption coefficient value corresponds to peak positions of P3HT samples plotted as a function of device thickness for experimental and theory (a) $d_{e}=10$

$$
\text { nm, (b) } d_{e}=125 \mathrm{~nm}, \text { (c) } d_{e}=500 \mathrm{~nm} \text { and (d) } d_{e}=1000 \mathrm{~nm} \text {. }
$$

A similar analysis has been done to P3HT: PCBM solar cells as shown in the graph above (Figure 4.8). The absorbance for peak photocurrent plotted as a function of device thickness is plotted along with model calculation to show the comparison. According to Figure 4.8, the charge collection length of $125 \mathrm{~nm}$ gives the best fit to the experimental data. It is clear evident that the increasing device thickness reduces the peak absorbance of the charge collection for both MDMO-PPV: PCBM and P3HT: PCBM solar cells. The normal exponential curve fitting is used for both cases to fit the experimental data points. By comparing charge collection lengths that fit with experimental data of P3HT and MDMO-PPV samples, concluding that the P3HT samples 
have a shorter $(125 \mathrm{~nm})$ collection length than the MDMO-PPV $(750 \mathrm{~nm})$ samples. This is probably due to the difference in morphology of two structures.

\subsection{Charge collection dependence on composition of P3HT: PCBM solar cells}

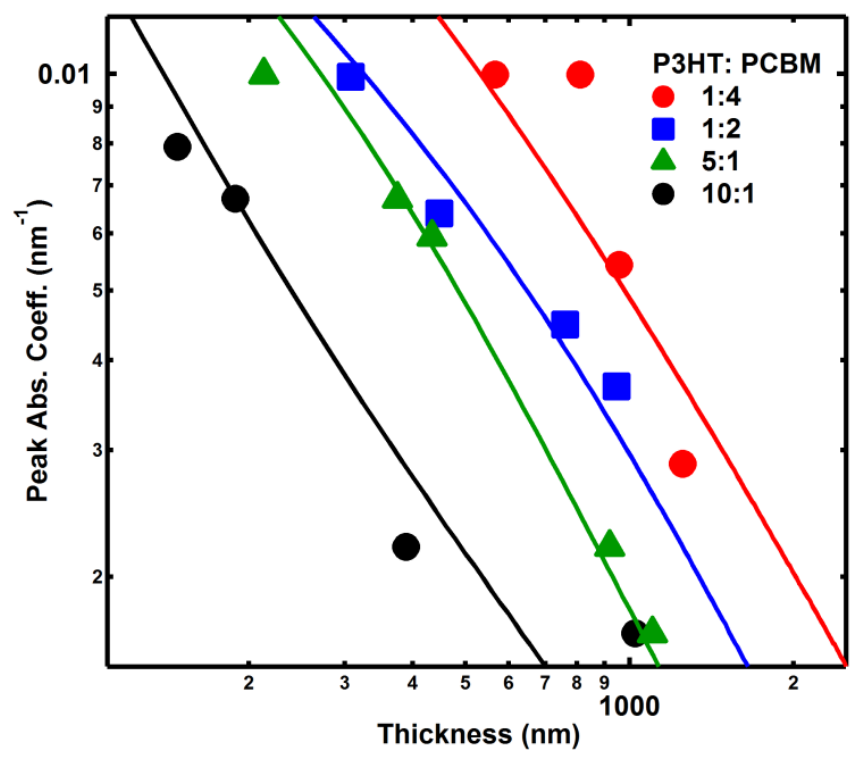

Figure 4.9: Peak absorption coefficient plotted as a function of device thickness of P3HT:

PCBM solar cells for different weight ratios.

This section describes the weight ration dependence on sample absorption in P3HT: PCBM solar cells. Fabrication procedure kept constant except the weight ratio between P3HT and PCBM has been changed in order to obtain weight ratios of 1(10 $\mathrm{mg}): 4(40 \mathrm{mg}), 1(10 \mathrm{mg}): 2(20 \mathrm{mg}), 5(50 \mathrm{mg}): 1(10 \mathrm{mg})$ and $10(10 \mathrm{mg}): 1(1 \mathrm{mg})$. Mixing was done using $1 \mathrm{ml}$ chlorobenzene. Then photocurrent spectra were measured by varying device thicknesses. Figure 4.9 shows the peak absorption coefficient plotted as a function of device thickness for all the weight ratios. There is a huge fluctuation of device thickness is observed due to the agglomeration of poor mixing of P3HT and PCBM. According to the graph, thinnest samples of almost all different weight ratios give the highest absorption coefficient for the peak photocurrent. And also increasing thickness 
reduces the peak absorption coefficient for all weight compositions. Other feature here is that the 1:4 weight ratio gives the highest device thicknesses which may be due to agglomeration and poor mixing of material on the surface. And the least device thicknesses occur for the weight ratio of 10:1 due to low material content.

This result is being compared with mobility measurements obtained in chapter 2. The graph 4.10 shows the electron mobility plotted as a function of PCBM\%. The charge collection length is also plotted for the comparison.

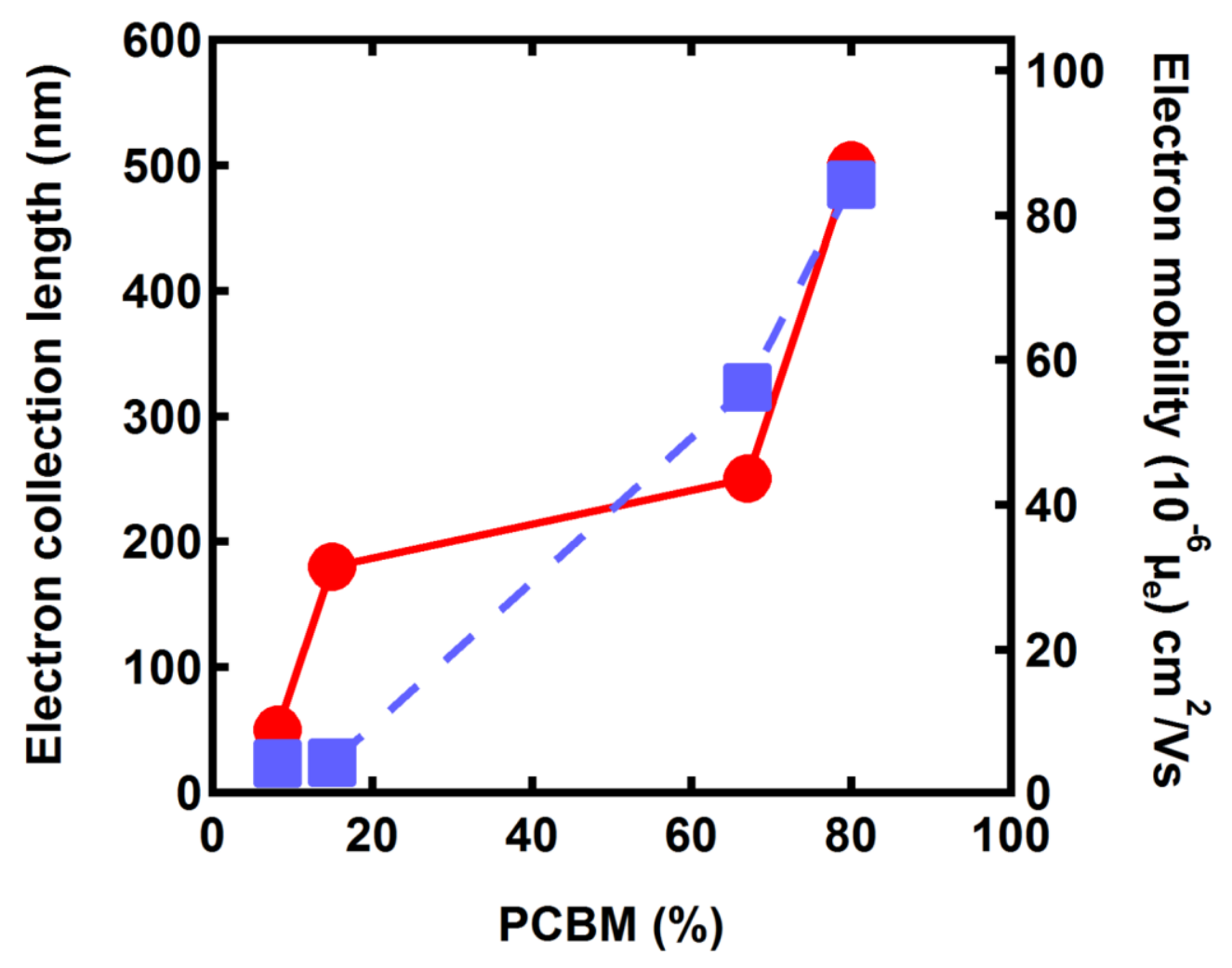

Figure 4.10: Electron mobility plotted as a function of PCBM\%. Electron collection length is also shown for the PCBM\%.

According to the above graph, electron mobility increases with increasing PCBM\%. This is an important conclusion that PCBM is the electron transporting material in these 
systems. This result is matched with the electron collection length derived from the model. Electron collection length increases with increasing PCBM\% which implies that longer electron collection lengths are obtained in high PCBM\%.

\subsection{Charge collection variation in front/back illumination}

A weight ratio of 5:4 of P3HT: PCBM was used for front/back illumination measurement. All the parameters in fabricating these devices kept the same in order to compare with other experiments. The light is illuminated from ITO side and it is called the front and when the illumination is done from $\mathrm{Al}$ side, it is called the back illumination. The peak of the absorption coefficients were measured with respect to the device thickness and plotted as shown below for the cases of $d_{e}>d_{h}$ and $d_{h}>d_{e}$. 

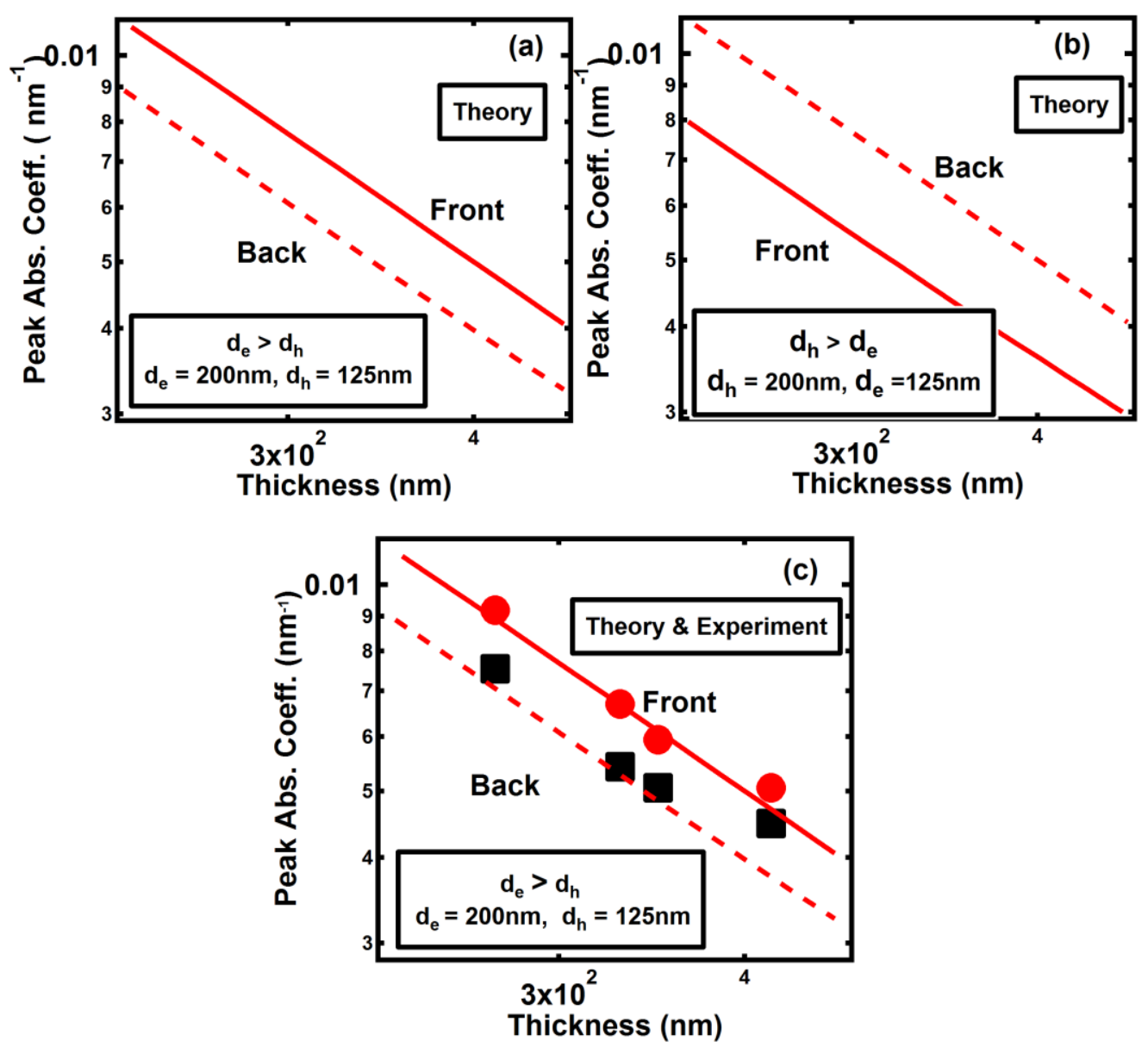

Figure 4.11: The absorption coefficient measured at the extracted photocurrent peak for the case of front and back illumination in P3HT/PCBM sample for $(a) d_{e}>d_{h},(b) d_{h}>d_{e}$ and (c) experiment $\left(d_{e}>d_{h}\right)$.

A curve fitting for the log-log plot is done using the model calculations that fits well the experimental data. The surface recombination distance for the fitting parameters kept at zero to easily compared. For front illumination, the absorption values are higher compared to that of back illumination. More importantly, the experimental data is fitted with the model giving the appropriate values of $d_{e}=200 \mathrm{~nm}$ and $d_{h}=125 \mathrm{~nm}$ for the case of front illumination. Then samples are flipped back side and the illumination is done 
from back side (Al side) of the samples to observe how the absorption coefficients are changed with thickness. The experimental data are fitted with the model calculation by interchanging the values of $d_{h}$ and $d_{e}$ (ie. for the back side illumination, $d_{h}=200 \mathrm{~nm}$ and $\mathrm{d}_{\mathrm{e}}=125 \mathrm{~nm}$ ). This is analogous with the model calculation results obtained in the theory chapter (Chapter 3 of this dissertation). Because when the illumination is done from the back side, the fraction of electron and holes collections interchanges each other. In other words, as described in previous chapter, the electron collection charge becomes holes and holes collection charge becomes electrons for the case of back illumination. The important feature here is that according to the figure 4.10(b), when $d_{h}>d_{e}$, the back illumination has higher absorption coefficient compared to that of front. This explains that the holes collection length plays the important role in charge collection for back illuminating devices. The same devices are used for this experiment in order to avoid the fluctuations in device thickness. 


\section{CHAPTER V: ANALYSIS OF AC PHOTOCURRENT SPECTRA}

This chapter covers three main measurements done with organic solar cells of P3HT: PCBM. The first part of the chapter covers AC measurements and the frequency dependence with a chopped white light source. Then the degradation of P3HT: PCBM solar cells will be discussed. Final section of this chapter is devoted to the new probing technique called capacitive photocurrent spectroscopy to investigate the dissociating efficiency of organic solar cells.

\subsection{AC photocurrent spectroscopy of organic solar cells}

Charge transfer time of the carriers generated by the photo-absorption depends on many factors. One of the important parameters is the collection length of the free carriers by the electrodes which most of previous chapters described. Collection length can also be estimated using the response time of the carriers. Hence it is worth to discuss the importance of this $\mathrm{AC}$ measurement to understand the properties of BHJ structures in organic solar cells.

In $\mathrm{AC}$ photocurrent measurements, the signal is detected only at a certain pulse width of light that is controlled by mechanical chopper (Figure 5.1). The signal is measured at modulation frequency of light pulse by the lockin amplifier. There are two reasons the signal is measured at chopping frequency. First is to make sure the signal measured is only due to light. This will avoid the electrical interference due to other 
frequency signals hence improving signal-to-noise ratio. The other reason is related with detection time of the carriers of the sample. The width of the light pulse incident on the device is inversely proportional to the frequency of light chopped. If the chopped frequency is $1 \mathrm{~Hz}$, duration of the detection time of the signal is one second. In other words, only the carriers generated and travelled to the electrodes in the sample within one second will only be collected by the system. In this way, it is possible to distinguish which carriers have shorter travel times and which have longer travel times that are not collected by the measurement. These travel times are determined by mobilities and the collection lengths of the carriers generated in the sample.

The Alternatiing current (AC) measurement at the open circuit voltage was measured for P3HT: PCBM organic solar cells using a low power white light QTH source. The schematic used to obtain AC photocurrent measurements is shown in Figure 5.1. The white light is resolved using a monochromator and mechanically chopped at different frequencies. These frequencies are sunk into a dual phase lockin amplifier and read through a digital voltmeter. The fabrication of the sample for this measurement was same as before and the sample is measured in an optical cryostat under inert atmosphere. The photovoltage is measured in P3HT samples at different chopping frequencies to observe the effect on thickness. 


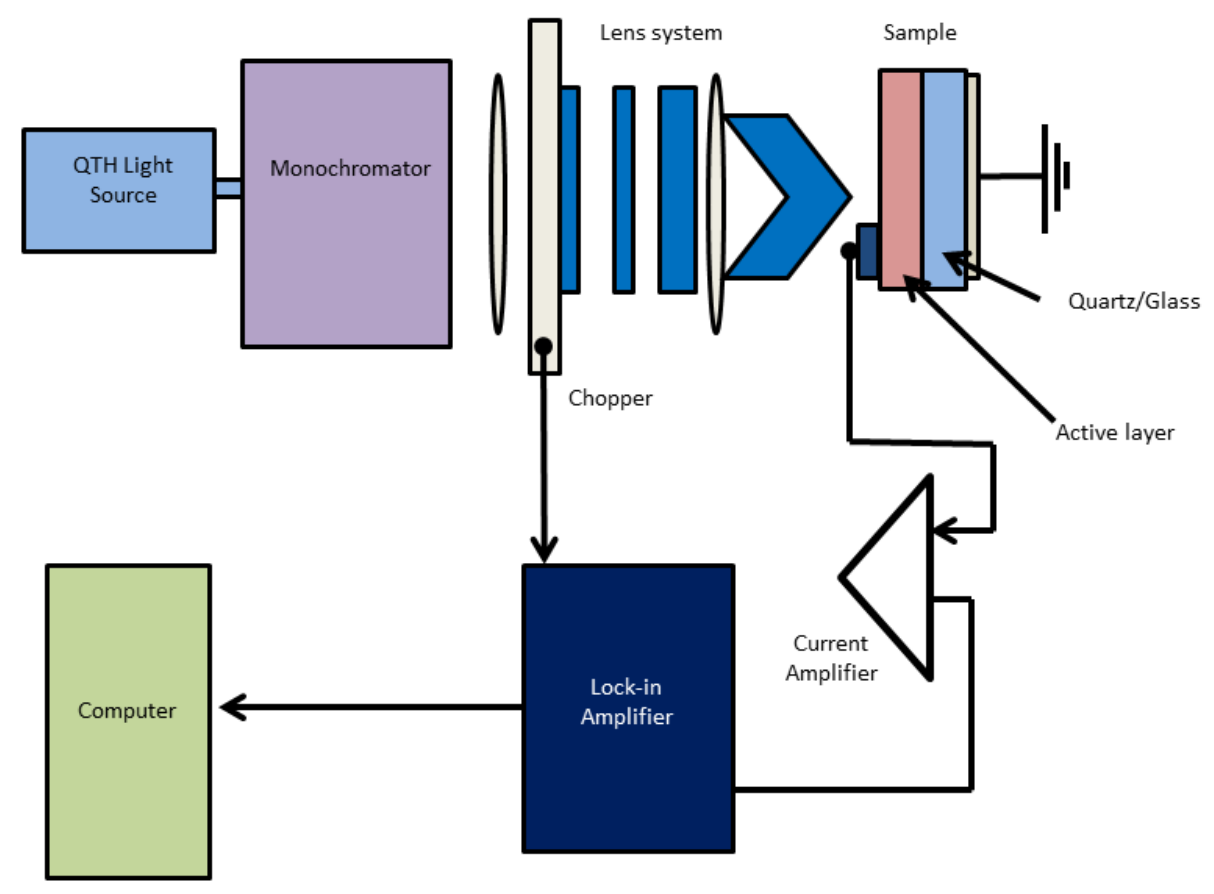

Figure 5.1: The schematic diagram of the AC photocurrent setup to measure the frequency dependence in organic bulk hetero junction solar cells.

The following figure shows the open circuit voltage $\left(\mathrm{V}_{\text {oc }}\right)$ dependence on wavelength for different chopping frequencies of P3HT: PCBM organic solar cells with 5:4 wt $\%$. The chopped light introduced in this case shows a clear dependence on photovoltage. When increasing the frequency, the signal decreases which explain that all the charges generated in the bulk are not collected by the electrodes due to short time periods. 


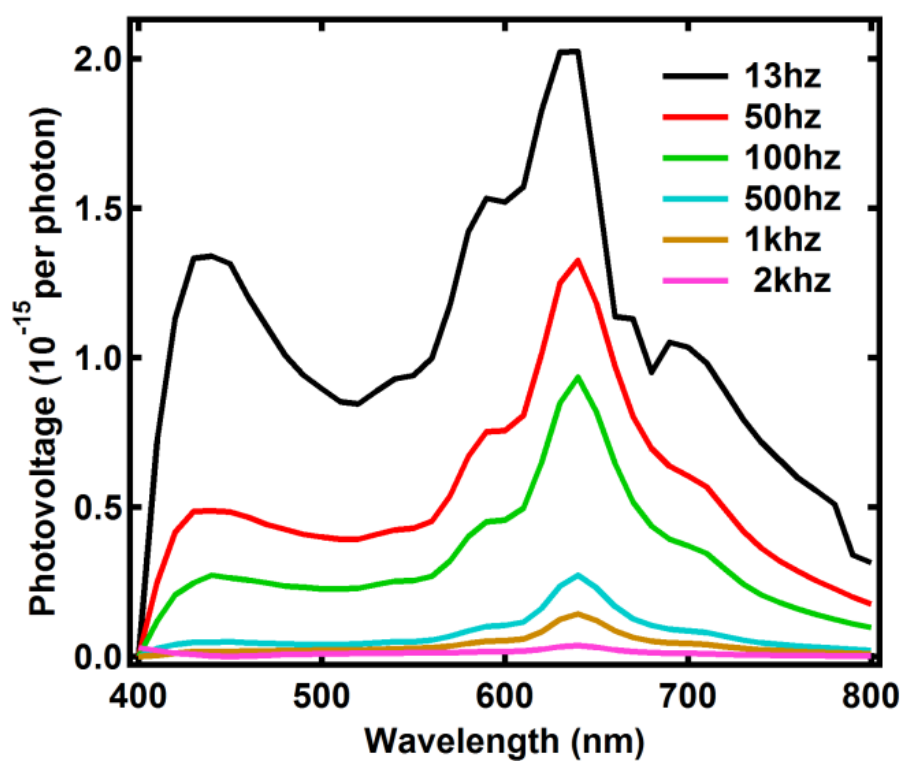

Figure 5.2. The AC photovoltage measured for P3HT: PCBM 5:4 wt\% samples for different frequencies.

This is for a device of $904 \mathrm{~nm}$ with $\mathrm{V}_{\text {oc }}$ signal is normalized per photon. Another important feature is that the high energy feature that is shown at $450 \mathrm{~nm}$ reduces as the frequency increases that suggest the high energy photogenerated carriers become neglected with short light pulses due to longer collection lengths and possible high order recombinations.

In order to observe the peak shift in the normalized photovoltage, the peak signal is plotted as a function of frequency for the samples of same 5:4 wt $\%$ ratio for four different devices which is shown in the graph below. The fitting is done to extract the frequency corresponds to maximum signal. 


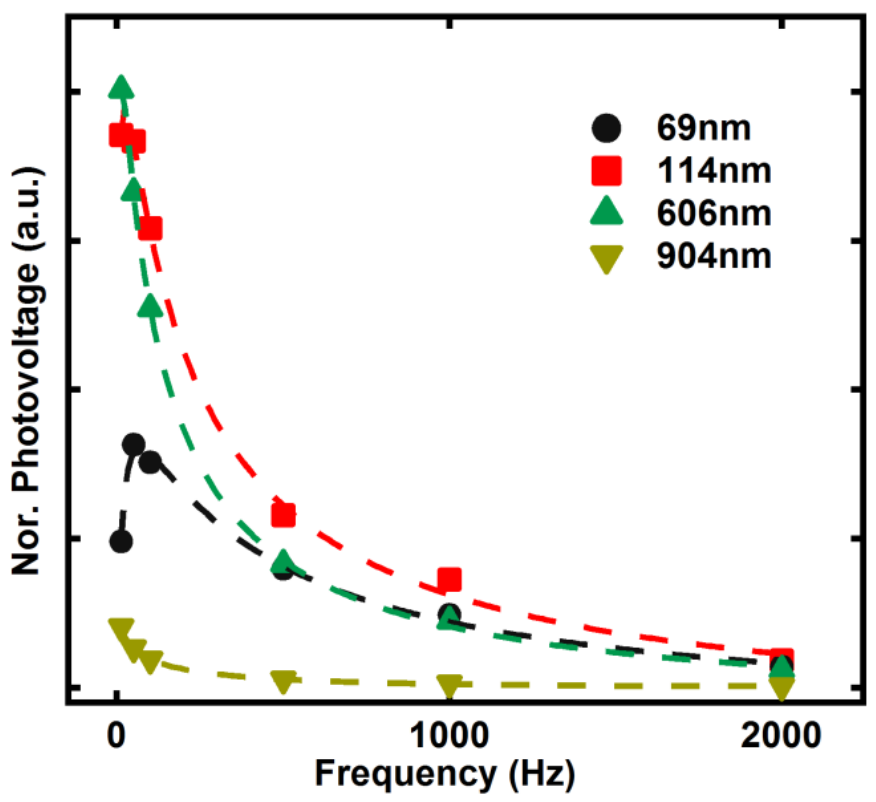

Figure. 5.3: The normalized photovoltage peak measured for P3HT: PCBM 5:4 wt\% samples for different frequencies.

It is clear evident that the peak signal decreases with increasing chopping frequency for all the thicknesses. Dash line shows the fittings to the experimental data. Using this fitting, the frequency corresponds to the maximum photovoltage is obtained. This measurement has been done with different wt $\%$ ratios in order to observe the frequency dependence on thickness. Following graph (Figure. 5.4) shows this frequency dependence for different wt\% ratio by taking the frequency corresponds to the peak signal of the fitting and plotted as a function of thickness. 


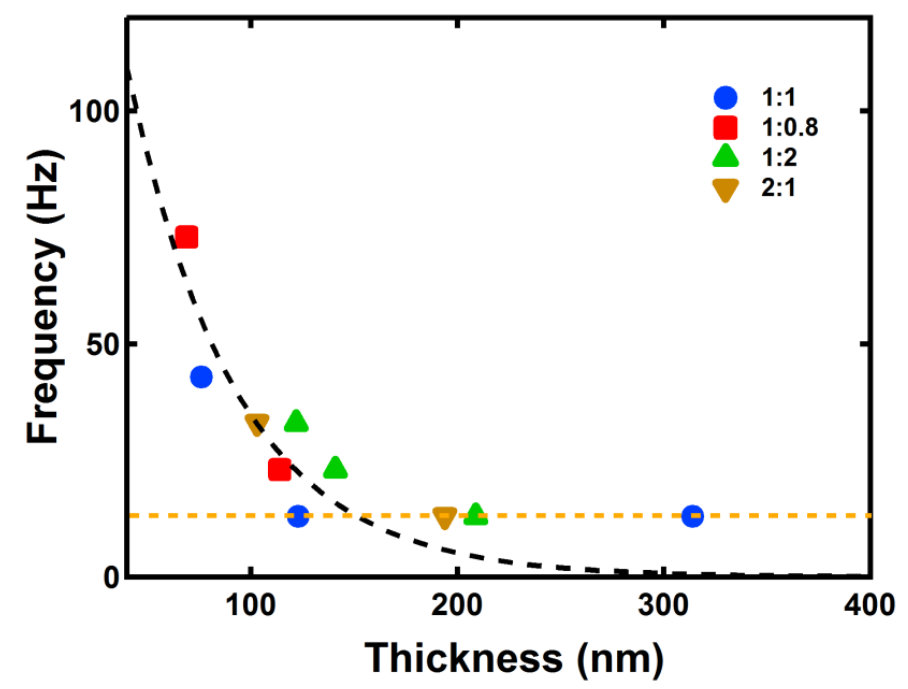

Fig. 5.4: The frequency corresponds to photovoltage peak plotted as a function of device thickness for P3HT: PCBM samples for different wt\% ratios.

The dash yellow line in the above graph is the minimum frequency used in the experiment $(13 \mathrm{~Hz})$. All devices peak frequencies limited by this value at higher thicknesses. According Figure 5.4, frequency corresponds to peak photovoltage decreases with increasing thickness and limit by the minimum frequency used in the experiment. It is clearly observed that for thin devices the $\mathrm{V}_{\text {oc }}$ signal become more sensitive to frequency while for thick devices this dependence can be neglected.

\subsection{Degradation measurements of P3HT: PCBM solar cells}

The stability of the organic solar cells has been a huge issue since its development. This is because of the active oxidation due to water/oxygen in the ambient atmosphere, reducing the short circuit current and open circuit voltage in the devices [6668]. A stability measurement of P3HT: PCBM organic solar cell done for a period of one week is shown here. The photocurrent spectrum was measured on the same device of P3HT: PCBM of 5:4 wt\% ratio for a thickness of 350nm and normalized for a fixed 
number of photons. In order to see the total photocurrent degradation, the integrated photocurrent were measured from 400 to $800 \mathrm{~nm}$ and normalized with respect to the peak value. The following graph shows the normalized integrated photocurrent for eight days.

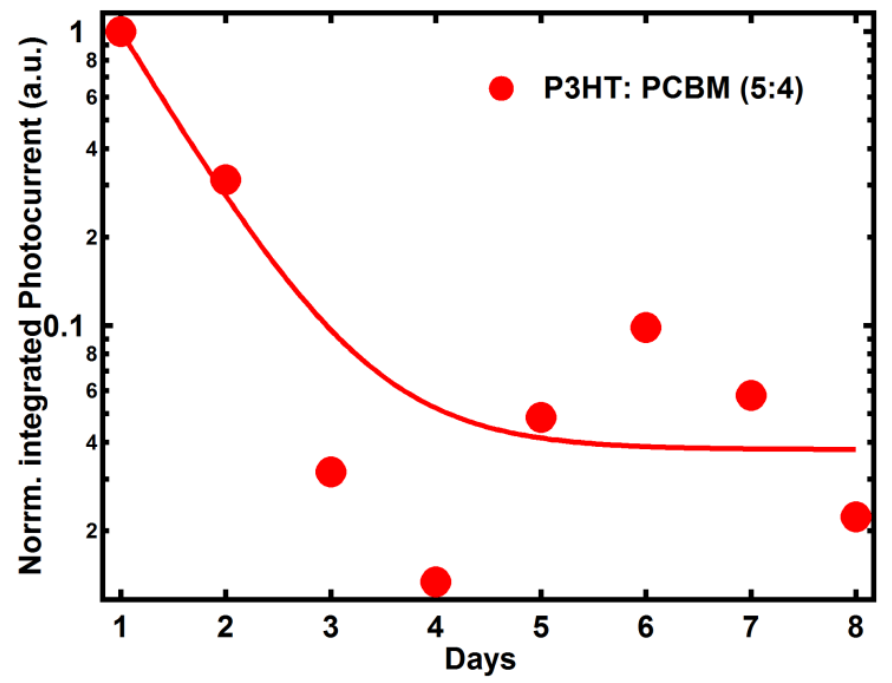

Figure 5.5: Normalized integrated photocurrent as a function of number of days for P3HT: PCBM solar cell.

According to the Figure 5.5, the integrated photocurrent was decreased with time. An exponential fit is also shown in the same graph. After three days the total photocurrent is reduced by 10 times is also evident from the graph.

Further analysis has been done for photocurrent degradation using different thicknesses shown in Figure 5.6. 


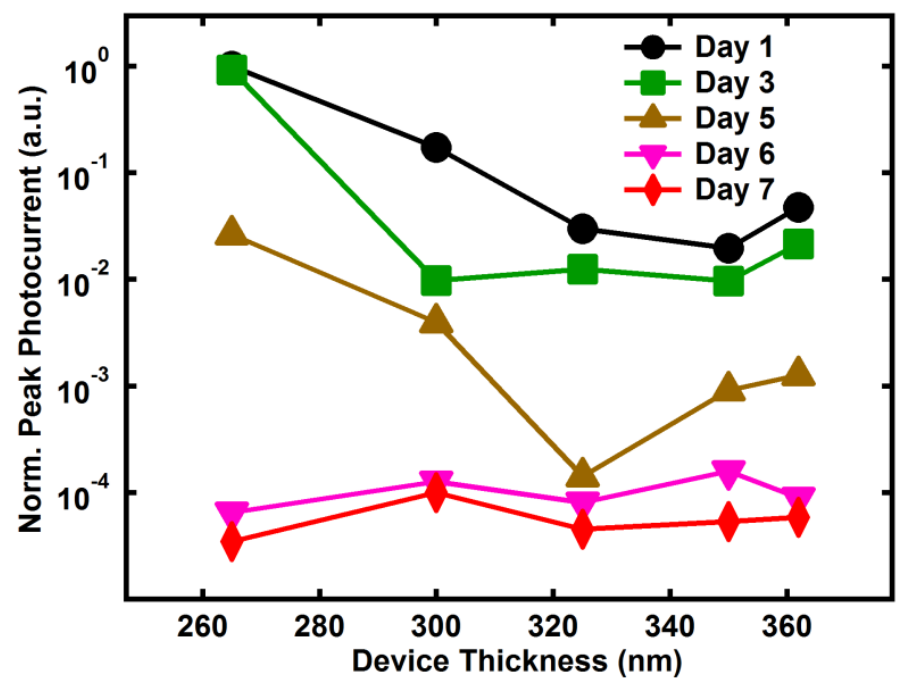

Figure 5.6: The Degradation photocurrent measurement of P3HT: PCBM solar cells with different thicknesses over a period of seven days.

Thickness was varied from $250 \mathrm{~nm}$ to $350 \mathrm{~nm}$ and the peak height of the photocurrent was measured for seven consecutive days. Then the data is plotted with respect to the maximum value as shown in Figure 5.6. The peak photocurrent decreases by $10^{4}$ within seven days. The samples were kept outside in ambient conditions to degrade for all seven days. It is also observed that after six days, the rate of degradation was reduced compared to first few days in all devices.

\subsection{Capacitive photocurrent Spectroscopy}

This section covers a unique technique used to measure the dissociation efficiency of band gap and sub-band gap exciton states of organic solar cells. This technique measures the potential energy generated due to photon absorption at the interface of bulk heterojunction solar cells. The device architecture for the capacitive photocurrent spectroscopy measurement is different to resistive structures used in normal photocurrent 
measurements. The active layer of P3HT: PCBM organic solar cell is spin coated on top of quartz/glass side of substrate and other side is coated with ITO. The ITO side is grounded by a copper block. Finally aluminum is coated on top of active layer in which the potential is measured with respect to the ground electrode. Quartz/glass acts as the dielectric medium of this capacitive structure (Figure 5.6).

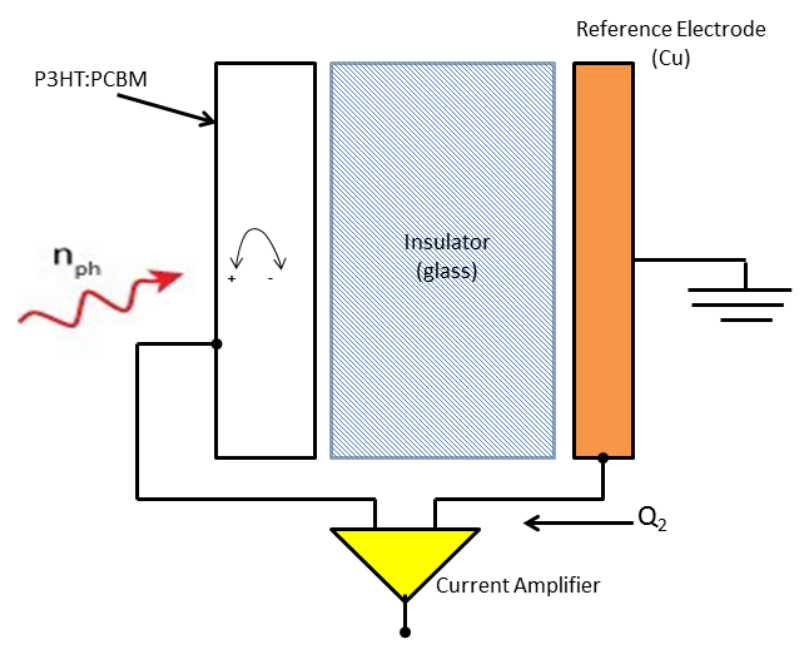

Figure 5.7 Schematic design of capacitive photocurrent spectroscopy.

When light is absorbed by the active layer of the cell, excitons generated and they dissociate at the interface. Once they dissociate an additional potential $\Delta \mathrm{V}$ is created with respect to the ground electrode. This potential created at the electrode due to light is given by $\frac{Q_{1}}{C_{1}}$ where $Q_{1}$ is the total charge per area generated at the exciton and $C_{1}$ is the capacitance of the charge separation. This potential is proportional to $Q_{1} d_{1}$ where $d_{1}$ is the exciton separation distance. If the e-h pairs remain bounded or recombined there is no 
potential change detected. Hence this technique is sensitive to exciton dissociation. Since the other end of the circuit is connected to zero potential ground electrode, a charge equals to potential of $\Delta \mathrm{V}$ will flow from copper electrode. This will occur to maintain equilibrium until the charge flow from the ground electrode equals to the potential generated due to light at the other electrode.

Suppose the amount of charge flown from the copper electrode is $\mathrm{Q}_{2}$. Then the amount of charge generated per unit area for a particular dielectric medium is given by

$$
Q_{2} \approx \frac{V}{d_{2}}
$$

Where $d_{2}$ is the charge separation distance between the bottom electrode and the active material exciton which is approximately equal to insulator (glass/quartz) thickness. By dividing Equation 5.1 by charge $\mathrm{Q}_{1}$,

$$
Q_{2} \approx Q_{1} \frac{d_{1}}{d_{2}}
$$

Assume the charge conversion efficiency of $0.01 \%$ with a $10^{16}$ number of incident photons and the glass thickness of $1 \mathrm{~mm}$. Also the charge separation distance of the exciton of $1 \mathrm{~nm}$ is assumed. Then a pico-amp (pA) photocurrent can be estimated from Equation 5.2 which is detected from the electrical circuit.

In practice a pulse or chopped light source is used and the resulting current is measured by a locking amplifier. The frequency of the chopped light sunk into locking amplifier so that only the signal of locked frequency will be measured. A better signal-tonoise ratio (SNR) will be achieved in this setup. Further review of this technique can be found in literature [69-71]. 

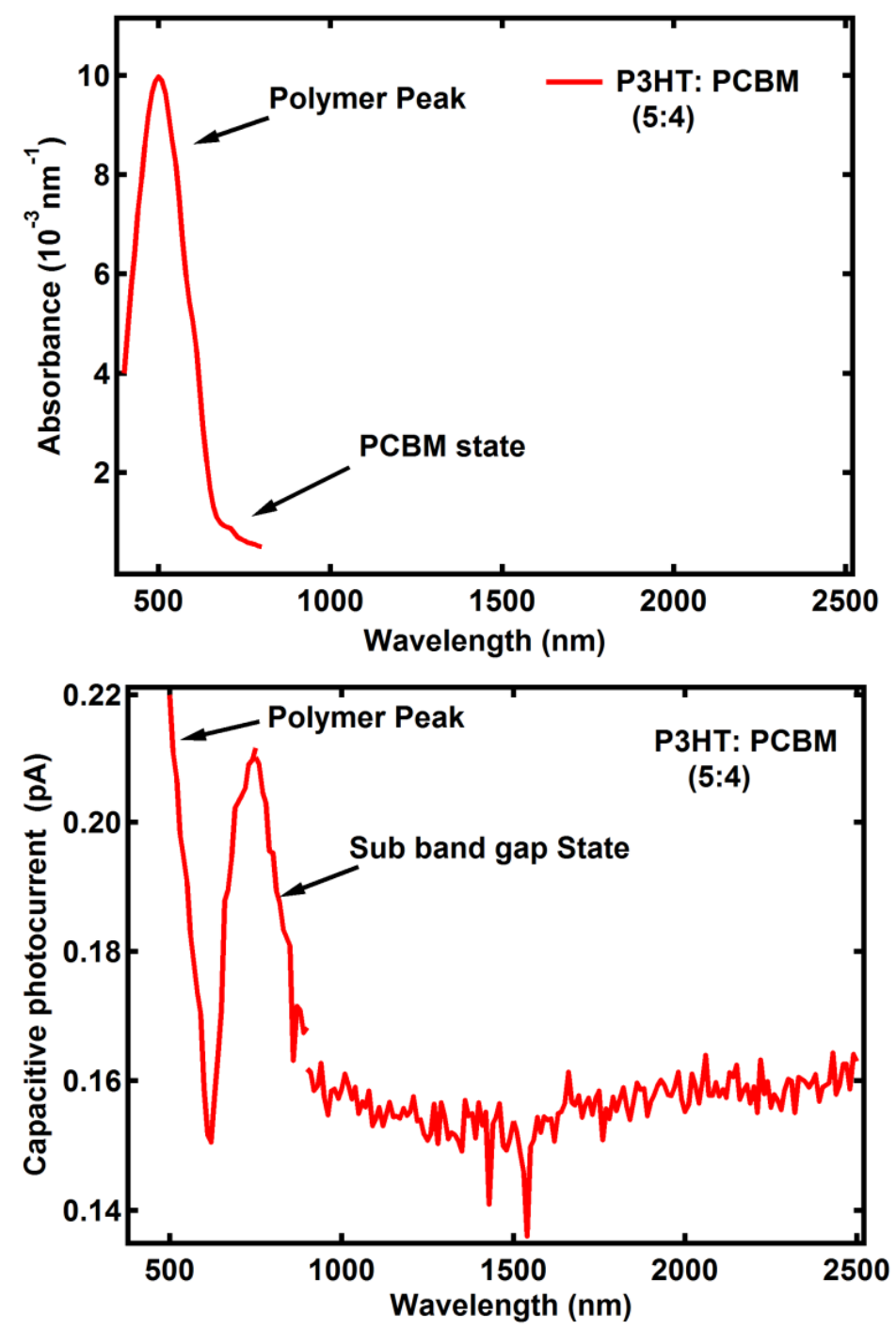

Figure 5.8 Capacitive photocurrent spectroscopy plotted along with the absorbance measurement of P3HT: PCBM of 5:4 wt\% ratio.

Figure 5.7 shows the capacitive measurement of P3HT: PCBM solar cell (bottom) plotted along the absorbance of the same structure (top). The polymer (P3HT) absorbance maximizes at $500 \mathrm{~nm}$ and started to drop off. A small shoulder is shown at $700 \mathrm{~nm}$ 
corresponds to singlet state (S1) of PCBM. This singlet state peak is enhanced by the capacitive photocurrent spectroscopy as shown in the bottom graph. It is worthwhile to investigate even though the high charge dissociation in PCBM evident in capacitive photocurrent results in low currents in these structures. Photobleaching experiments have been done to MDMO-PPV: PCBM systems suggested [70] this singlet state acts as a trap state in charge transfer to Aluminum that explains the lack of contribution to photocurrent extraction from PCBM. Further it is been suggested that filtering out this state can be used to enhance the efficiency in solar cells. 


\section{CHAPTER VI: CONCLUSIONS AND DISCUSSION}

Low efficiency of organic polymer solar cells has been a major issue since its initial development. It is believed that lack of understanding in several major steps including charge generation, dissociation, transfer mechanism, recombination and charge extraction could have attributed to this difficulty in enhancing efficiencies. This dissertation focuses on expanding the current understanding of charge transfer, recombination and extraction mechanisms in organic polymer solar cells.

Addressing these issues began from an analysis of the photocurrent dependence on thickness of the active materials used in organic solar cells. Preliminary measurements suggest that the total photocurrent obtained from a solar cell device has an inverse relationship with the thickness of its active material even though they absorb more percentage of light with respective to the solar spectrum. Due to this effect, thick solar cells have not been widely used in optoelectronic applications. A systematic study was conducted on the photocurrent spectra of organic polymer solar cell structures in order to examine its dependence to thickness. Preliminary experimental results suggest a red shift of the peak of the normalized photocurrent with respect to the device thickness of two major material systems used in organic polymer solar cells, namely MDMO-PPV: PCBM and P3HT: PCBM. In order to understand these results further, a theoretical model has been implemented in Chapter 3 including charge carrier collection length, 
absorption variation and surface recombination. This model has led to estimate two important parameters associated with charge transfer, recombination and extraction mechanisms of organic solar cells. They are the charge collection and surface recombination lengths.

Chapter 3 mainly demonstrates the derivation of the model of charge extraction as a function of device thickness. This was obtained to fit the experimental photocurrent measurements presented in chapter four. The electron collection lengths were estimated for P3HT and MDMO-PPV solar cell devices. In the preliminary analysis, DC photocurrent was measured by resolved white light from a monochromator with varying active layer thicknesses. For P3HT devices, the photocurrent of thinnest device of $62 \mathrm{~nm}$ matches the absorbance of that material. When thickness increased, the peak of the polymer shifted to longer wavelength region of visible spectrum that matches with the photoluminescence. This behavior was observed in MDMO-PPV devices too. Again the thinnest device of $125 \mathrm{~nm}$ matches with the absorbance of MDMO-PPV and increase of thickness shifts to longer wavelength region of the spectrum that aligns with photoluminescence. A larger thickness variation was obtained in MDMO-PPV devices than that of $\mathrm{P} 3 \mathrm{HT}$ devices due to lower absorption coefficients associated with MDMOPPV. Theoretical model was introduced to fit the experimental data that measures the carrier extraction from front and back electrodes as a function of device thickness.

Model also showed a red shift of the carrier collection for electrons with increasing thickness when the illumination was done from front side. There was no red shift of the holes collection observed with varying thickness for front illuminating devices. According to the model calculation, electron collection photocurrent value was 
low compared to that of the holes collection photocurrent. Hence, electrons, smaller of carrier type determine the overall photocurrent of the solar cell device for front illumination. As a further analysis, this shift was analyzed with different charge collection lengths in order to fit with the experimental data to determine charge collection length for the organic solar cells. In chapter four, peak absorption coefficient was plotted as a function of the device thickness for MDMO-PPV and P3HT solar cells with different charge collection lengths. P3HT and MDMO-PPV devices yielded a charge collection length of $100 \mathrm{~nm}$ and $750 \mathrm{~nm}$ respectively that fits well with experimental data. MDMOPPV devices displayed a larger collection length than that of P3HT devices. This implicate that carrier collection in MDMO-PPV devices is more efficient than that of the P3HT devices. On the other hand, larger collection lengths increase the probability of recombination during their journeys to the respective electrodes.

Back illumination was conducted to observe the dependence of holes collection charge on thickness of these devices. Experimental data indicated that the front illumination has a higher absorption by the material compared to that of the back illumination. Model fittings with experimental data suggested that the electron collection charge for these devices is higher than that of holes. When the holes collection charge was higher than electrons, the back illumination resulted in a higher absorption effect by the material. This clearly indicates that holes collection plays a major role in the back illuminating devices while electrons play the major role in the front illuminating devices.

Different interfacial layers were introduced to observe the effect on surface recombination length for P3HT solar cell devices. PEDOT-PSS and thin layer of P3HT was used for interfacial layers. Model was fitted with the experimental data by keeping 
the electron and holes collection lengths constant and varying the surface recombination length to determine which interfacial layers give the least surface recombination length. Experimental data suggested that the device with PEDOT-PSS yields the least surface recombination length of $0 \mathrm{~nm}$. Longer surface recombinations are showed for the devices with P3HT and without interfacial layer. This suggests that interfacial layer of PEDOTPSS should be used to optimize the carrier collection efficiency by minimizing the surface recombination. Hence this model provides an efficient method to find the surface recombination, and thus useful to choose a suitable interfacial layer to optimize the device efficiency.

AC photocurrent measurements were conducted to investigate how sensitive the frequency of the chopped AC light on the thickness of P3HT solar cells. These experiments revealed that the thinner samples are more sensitive to AC light while thicker samples are more sensitive to DC light as lowering the frequency reduces the AC photocurrent. This pattern mainly occurred due to the decrease of response time with increasing short light pulses. Also, the frequency of the peak photovoltage dependence on device thickness suggests that ultra-short pulse light can be used to further improve the voltages of thinner devices. Hence the devices with short carrier collection lengths will improve the efficiency of thinner devices, while long carrier collection lengths will improve the efficiency of thicker devices. This suggests that thicker devices can be used in solar cell applications with materials of longer carrier collection lengths.

Degradation measurements have been done with P3HT: PCBM solar cells in order to investigate the stability on device thickness. The degradation of short circuit current of solar cell devices has been measured for over a period of seven days for 
different thicknesses. The results suggested that the degradation occurs in all the devices regardless of the device thickness. The devices have been shown stability after five days with respect to the initial degradation rate. The degradation rate is fitted well with exponential decay function.

Capacitive photocurrent technique was used to investigate the sub bandgap states in P3HT: PCBM solar cells using a low power white light source. The results suggest that the huge enhancement in capacitive photocurrent measurement corresponds to the sub band gap states in addition to the main absorbance peak. This clearly indicate that the sub bang gap states in P3HT: PCBM solar cells have high charge carrier dissociation efficiency even with low power white light that may not contribute to the overall photocurrent generation. Furthermore populating more carriers or introducing windowing layer to extract these charge carriers in sub band gap states can be used to enhance the efficiency of organic solar cells.

\section{Future work:}

As demonstrated in the model and the experimental data, this method of charge carrier extraction can be used to identify the materials with large collection lengths which could be potentially useful in optimizing the device efficiency of organic solar cells. Different interfacial layers can be used with new materials to analyze the surface recombination lengths which will lead to an efficient interfacial layer and hence a better charge collection efficiency.

A systematic photocurrent study can be carried out with different wt $\%$ ratios of organic solar cells. The model developed in this dissertation can be used to identify the 
appropriate charge collections and mobilities that will lead to an optimized wt\% composition in organic bulk heterojunction solar cells.

Capacitive photocurrent spectroscopy results identify a high dissociation from the sub bandgap states in these organic solar cells. This will open up a whole new avenue to enhance the charge collections of organic solar cells by identifying the sub bandgap states which cannot be detected using the existing characterization methods. 


\section{REFERENCES}

1. Yu, G., ;Heeger,A.,J., Charge Seperation and Photovoltaic Conversion in polymer composites with internal donar/acceptor heterojunctions. Journal of Applied Phyiscs, 1995. 78(7).

2. Chapin, D.M., C.S. Fuller, and G.L. Pearson, A New Silicon $p-n$ Junction Photocell for Converting Solar Radiation into Electrical Power. Journal of Applied Physics, 1954. 25(5): p. 676-677.

3. Tang, C.W., Two-layer organic photovoltaic cell. Applied Physics Letters, 1986. 48(2): p. 183.

4. Jin Young Kim, 2 Kwanghee Lee,1,2* Nelson E. Coates, 1 Daniel Moses, 1 and M.D. Thuc-Quyen Nguyen, 1 Alan J. Heeg, Efficient Tandem Polymer Solar Cells Fabricated by All-Solution Processing. SCIENCE, 2007. 317(13): p. 222-224.

5. Li, G., et al., High-efficiency solution processable polymer photovoltaic cells by self-organization of polymer blends. Nature Materials, 2005. 4(11): p. 864-868.

6. Francis Garnier, R.H., Abderrahim Yassar, and P. Srivastava, All-Polymer FieldEffect Transistor Realized by Printing Techniques. Science, 1994. 265: p. 16841686.

7. Thompson, B.C. and J.M. Frechet, Polymer-fullerene composite solar cells. Angewandte Chemie, 2008. 47(1): p. 58-77. 
8. Swaraj, S., et al., Nanomorphology of bulk heterojunction photovoltaic thin films probed with resonant soft X-ray scattering. Nano Lett, 2010. 10(8): p. 2863-9.

9. Hoppe, H. and N.S. Sariciftci, Polymer Solar Cells, in Photoresponsive Polymers II, S. Marder and K.-S. Lee, Editors. 2008, Springer Berlin Heidelberg. p. 1-86.

10. G. Grancini, et al., Hot exciton dissociation in polymer solar cells. Nature Materials, 2013. 12(1): p. 29-33.

11. Hoppe, H. and N.S. Sariciftci, Organic solar cells: An overview. Journal of Materials Research, 2004. 19(7): p. 1924-1945.

12. Keevers, M.J. and M.A. Green, Absorption edge of silicon from solar cell spectral response measurements. Applied Physics Letters, 1995. 66(2): p. 174.

13. Servaites, J.D., M.A. Ratner, and T.J. Marks, Organic solar cells: A new look at traditional models. Energy \& Environmental Science, 2011. 4(11): p. 4410.

14. Gregg, B., Excitonic Solar Cells. Journal of Physical Chemistry B, 2003. 107(20): p. $4688-4698$.

15. H.W. Kroto, J.W.H., S.C. O'Brien, R.F.Curl, R.E. Smalley, C60:Buckministerfullerene. Nature, 1985. 318(6042): p. 162-163.

16. W. Kratschmer, L.W.L., K. Fostiropoulos, D.R. Huffman, Solid C60: a new form of carbon. Nature, 1990. 347: p. 354-357.

17. Jan C. Hummelen, B.W.K., F. LePeq, Fred Wudl, Jie Yao and Charles L. Wilkins, Preparation and Characterization of Fulleroid and Methanofullerene Derivatives. The Journal of Organic Chemistry 1995. 60( 3): p. 532-538.

18. Pfuetzner, S., et al., Improved bulk heterojunction organic solar cells employing C[sub 70] fullerenes. Applied Physics Letters, 2009. 94(22): p. 223307. 
19. Yao, Y., et al., Effects of C[sub 70] derivative in low band gap polymer photovoltaic devices: Spectral complementation and morphology optimization. Applied Physics Letters, 2006. 89(15): p. 153507.

20. Gregg, B.A. and M.C. Hanna, Comparing organic to inorganic photovoltaic cells: Theory, experiment, and simulation. Journal of Applied Physics, 2003. 93(6): p. 3605 .

21. Deibel, C., T. Strobel, and V. Dyakonov, Role of the charge transfer state in organic donor-acceptor solar cells. Adv Mater, 2010. 22(37): p. 4097-111.

22. Veldman, D., S.C.J. Meskers, and R.A.J. Janssen, The Energy of Charge-Transfer States in Electron Donor-Acceptor Blends: Insight into the Energy Losses in Organic Solar Cells. Advanced Functional Materials, 2009. 19(12): p. 1939-1948.

23. Street, R.A. and M. Schoendorf, Interface state recombination in organic solar cells. Physical Review B, 2010. 81(20).

24. Vithanage, D.A., et al., Visualizing charge separation in bulk heterojunction organic solar cells. Nat Commun, 2013. 4.

25. Veaceslav Coropceanu, J.r.m.C., $\$, \dagger$ Demetrio A. da Silva Filho, $\uparrow$ Yoann Olivier, $\$$ Robert Silbey,\# and and J.-L. Bre’das*, Charge Transport in Organic Semiconductors. Chemical Review, 2007. 107(4): p. 926-952.

26. Peumans, P., A. Yakimov, and S.R. Forrest, Small molecular weight organic thinfilm photodetectors and solar cells. Journal of Applied Physics, 2003. 93(7): p. 3693. 
27. Irwin, M.D., et al., p-Type semiconducting nickel oxide as an efficiencyenhancing anode interfacial layer in polymer bulk-heterojunction solar cells. Proceedings of the National Academy of Sciences, 2008. 105(8): p. 2783-2787.

28. Dong-Ho Kim*, J.-W.K., Hye-Ri Kim, Yong-Jin Kang, Sun-Young Park, and Yong-Soo Jeong, Sputter deposited p-type nickel oxide thin films as an anode buffer layer in organic solar cells, in Photovoltaic Specialists Conference (PVSC), 2011 37th IEEE 2011. p. 001173 - 001175.

29. Shrotriya, V., et al., Transition metal oxides as the buffer layer for polymer photovoltaic cells. Applied Physics Letters, 2006. 88(7): p. 073508.

30. Hau, S.K., et al., Air-stable inverted flexible polymer solar cells using zinc oxide nanoparticles as an electron selective layer. Applied Physics Letters, 2008. 92(25): p. 253301.

31. Waldauf, C., et al., Highly efficient inverted organic photovoltaics using solution based titanium oxide as electron selective contact. Applied Physics Letters, 2006. 89(23): p. 233517.

32. Liu, Z. and E.-C. Lee, Efficiency improvement in fullerene-layer-inserted organic bulk-heterojunction solar cells. Journal of Applied Physics, 2012. 111(2): p. 023104.

33. M. Granstro"m*, K.P., A. C. Arias*, A. Lux ${ }^{\dagger}$, and M.R.A.R.H. Friend, Laminated fabrication of polymeric photovoltaic diodes. Nature, 1998. 395(6699): p. 257-259.

34. Gunes, S., H. Neugebauer, and N.S. Sariciftci, Conjugated polymer-based organic solar cells. Chem Rev, 2007. 107(4): p. 1324-38. 
35. Gang Li, R.Z.a.Y.Y., Polymer solar cells. Nature Photonics, 2012. 6: p. 153-161.

36. Brabec, C.J., et al., Effect of LiF/metal electrodes on the performance of plastic solar cells. Applied Physics Letters, 2002. 80(7): p. 1288.

37. I. Riedel, J.P., V. Dyakonov, L. Lutsen, D. Vanderzande, J. C. Hummelen Effect of temperature and illumination on electrical charateristics of polymer fullerene bulk heterojunction solar cells. Adv. Funct. Mater., 2004. 14(1): p. 38-44.

38. Pivrikas, A., et al., Bimolecular Recombination Coefficient as a Sensitive Testing Parameter for Low-Mobility Solar-Cell Materials. Physical Review Letters, 2005. 94(17).

39. Diebel, C., Charge Carrier Dissociation and Recombination in Polymer Solar Cells. Phys. Status Solidi A, 2009. 206: p. 2731.

40. Abkowitz, M., J.S. Facci, and J. Rehm, Direct evaluation of contact injection efficiency into small molecule based transport layers: Influence of extrinsic factors. Journal of Applied Physics, 1998. 83(5): p. 2670.

41. Campbell, A.J., D.D.C. Bradley, and H. Antoniadis, Quantifying the efficiency of electrodes for positive carrier injection into poly(9,9-dioctylfluorene) and representative copolymers. Journal of Applied Physics, 2001. 89(6): p. 3343.

42. Charlesby, G.P.O.a.A., On charge-carrier trapping in insulating solids as investigated by the space-charge-limited current (SCLC) technique: a cautionary note. J. Phys. C: Solid State Phys, 1974. 7: p. L400-L402.

43. Lampert, M.A.a.P.M., Current injection in solids. Academic Press. 1970: New York. 
44. Many, A. and G. Rakavy, Theory of Transient Space-Charge-Limited Currents in Solids in the Presence of Trapping. Physical Review, 1962. 126(6): p. 1980-1988.

45. Poplavskyy, D. and J. Nelson, Nondispersive hole transport in amorphous films of methoxy-spirofluorene-arylamine organic compound. Journal of Applied Physics, 2003. 93(1): p. 341.

46. Jingyao, S., Charge transport and excited states in organic semiconductors, 2010, University of London.

47. Kepler, R., Charge Carrier Production and Mobility in Anthracene Crystals. Physical Review, 1960. 119(4): p. 1226-1229.

48. LeBlanc, O.H., Electron Drift Mobility in Liquid n-Hexane. The Journal of Chemical Physics, 1959. 30(6): p. 1443.

49. Sachetan M Tuladhar, D.P., Stelios A. Choulis, James R. Durrant, Donald D.C. Bradley, Jenny Nelson, Ambipolar charge transport in films of Methonafullerene and Poly(phenylenevinylene) Methanofullerene Blends. Advanced Functional Materials, 2005. 15: p. 1171-1182.

50. Yasuhiko Shirota*, a.H.K., Charge Carrier Transporting Molecular Materials and Their Applications in Devices. Chemical Review, 2007. 107: p. 953-1010.

51. Wallace, J.U., Carrier Mobility in Organic Charge Transport Materials:Methods of Measurement, Analysis, and Modulation, in Department of Chemical Engineering2009, University of Rochester.

52. Hoppe H., S.N.S., Oranic Solar Cells:An overview. Journal of Mater. Res, 2004. 19(7). 
53. J.J.M. Halls, C.A.W., N.C. Greenham, E.A. Marsgella, R.H. Friend, S.C.Moratti, A.B. Holmes Efficient photodiodes from interpenetrating polymer networks. Nature, 1995. 376: p. 498-500.

54. McGehee, K.M.C.a.M.D., Conjugated Polymer Photovoltaic Cells. Chem Rev, 2004.

55. Krebs, F.C., Polymer Photovoltaics: A practical approach, ed. F.C. Krebs. 2008: SPIE Press.

56. Ma, W., et al., Thermally Stable, Efficient Polymer Solar Cells with Nanoscale Control of the Interpenetrating Network Morphology. Advanced Functional Materials, 2005. 15(10): p. 1617-1622.

57. Shah, H.M., Excition generation and dissociation mechanisms in organic bulk heterojunction solar cell materials, in Electrical Engineering 2012, University of Louisville: Louisville. p. 152.

58. Sariciftci, C.W.a.N.S., Low bandgap polymers for photon harvesting in bulk heterojunction solar cells. Journal of Materials Chemistry, 2004(14): p. 1077 1086 .

59. Hoppe, H., S. Shokhovets, and G. Gobsch, Inverse relation between photocurrent and absorption layer thickness in polymer solar cells. physica status solidi (RRL) - Rapid Research Letters, 2007. 1(1): p. R40-R42.

60. Hallermann, M., et al., Charge Transfer Excitons in Polymer/Fullerene Blends: The Role of Morphology and Polymer Chain Conformation. Advanced Functional Materials, 2009. 19(22): p. 3662-3668. 
61. Colladet, K.F., S.; Cleij, T, J,; Lutsen, L.; Gelan, J.; Vanderzande, D., Low Band Gap Donor-Acceptor Conjugated Polymers toward Organic Solar Cells Applications. Macromolecules, 2007. 40(1): p. 65-72.

62. Goh, C., et al., Molecular-weight-dependent mobilities in regioregular poly(3hexyl-thiophene) diodes. Applied Physics Letters, 2005. 86(12): p. 122110.

63. Chirvase, D., et al., Temperature dependent characteristics of poly(3 hexylthiophene)-fullerene based heterojunction organic solar cells. Journal of Applied Physics, 2003. 93(6): p. 3376.

64. Kristof Colladet, S.F., $\uparrow$ Thomas J. Cleij, $\uparrow$ Laurence Lutsen, $\uparrow$ Jan Gelan, $\uparrow$ and and L.H.N. Dirk Vanderzande, Helmut Neugebauer, and Serdar Sariciftci, Aranzazu Aguirre, Griet Janssen, and Etienne Goovaerts, Low Band Gap Donor-Acceptor Conjugated Polymers toward Organic Solar Cells Applications. Macromolecules, 2007. 40(1): p. 65-72.

65. Abeyweera, B.K. and B.W. Alphenaar, Thickness dependent red shift of the photocurrent spectrum in bulk heterojunction solar cells. Applied Physics Letters, 2013. 102(4): p. 041113.

66. Singh, V., et al., Degradation in bulk heterojunction organic solar cells: changes in electrode interface and reduction in the occupation probability of the interface states. Journal of Polymer Research, 2012. 19(6).

67. Kawano, K., et al., Degradation of organic solar cells due to air exposure. Solar Energy Materials and Solar Cells, 2006. 90(20): p. 3520-3530. 
68. Yang, W., Y. Yao, and C.-Q. Wu, Mechanisms of device degradation in organic solar cells: Influence of charge injection at the metal/organic contacts. Organic Electronics, 2013. 14(8): p. 1992-2000.

69. Mohite, A.D., et al., Observation of the triplet exciton in EuS-coated singlewalled nanotubes. Nat Nanotechnol, 2009. 4(7): p. 425-9.

70. Shah, H.M., et al., Photovoltage bleaching in bulk heterojunction solar cells through population of the singlet exciton. Applied Physics Letters, 2010. 97(26): p. 263301.

71. Mohite, A.D., et al., Exciton Dissociation and Stark Effect in the Carbon Nanotube Photocurrent Spectrum. Nano Letters, 2007. 8(1): p. 142-146. 


\section{CURRULUM VITAE}

\section{BUDDIKA K ABEYWEERA}

776 David Fairleigh ct, Apt. 03, Louisville, KY 40217.

Email: e2buddika@gmail.com

Phone: (502)2967901

\section{RESEARCH INTERESTS}

Nano-electronics, Micro/nano-fabrication, characterization (electrical and optical) of nano-materials, Renewable energy research with emphasis on Organic photo-voltaic devices, electrodes and sensors, Carbon electronics and optoelectronics.

\section{PROFESSIONAL PROFILE}

- Pursuing the Ph.D. in Electrical and Computer Engineering under Prof. Bruce W. Alphenaar.

- 6+ years of hands on experience in applied and fundamental research in the field of Micro/Nano-scale device fabrication, Optical characterization, Material Science, Semi-conductor Physics, Cryogenics and Vacuum technologies.

- Proven research track with 4 peer reviewed journal papers, 2 Oral and 5 poster presentations at various international conferences (SPIE, MRS, APS, KYEPSCoR).

- Supervision and training of M.S. and new Ph.D. students for PVD systems (Ebeam/Thermal evaporation) and Optical characterization techniques such as IPCE systems, AFM, SEM and Spectra Physics femto-second pulse laser system with tunable OPA capabilities.

- Proven ability to work both independently and as a part of multi-disciplinary research teams.

\section{EDUCATION}

Ph.D. Electrical Engineering (May 2009- Dec 2013)

University of Louisville, Louisville, KY under Prof. Bruce W. Alphenaar

M.S. Physics (Aug 2007 - May 2009)

University of Louisville, Louisville, KY under Prof. Gamini U. Sumanasekera 
BSc. Physics (Hons.) (Feb 2002 - June 2006)

University of Peradeniya, Sri Lanka

\section{ACCOMPLISHMENTS}

- Designed and constructed a complete material characterization system that controls a monochromater to obtain the Photocurrent Spectrum of Solar cells and calculate the IPCE (QE\%). This low budget system enables an accurate data acquisition and faster sample characterization.

- First to demonstrate the variation in photocurrent with thickness in organic solar cells and the limitations of the efficiency of organic solar cells.

- Demonstrated an innovative optical-electronic technique: Capacitive Photocurrent Spectroscopy (CPS) that is sensitive to sub band-gap states in organic bulk heterojunction solar cells.

- Designed and assembled a Thermal Evaporator system that enables rapid thin film deposition for solar cell applications.

- Designed and assembled a surface characterization system that allows to sense different gasses under controlled pressure by carbon nanostructures. This system acts as a chemical sensor for identifying gases that use in chemical weapons.

- Designed a new overlapping technique for fabricating Solar Cell module that enhances the device productivity and yield by $80 \%$.

- Maintained and upgraded LabVIEW based data acquisition systems for sample characterization.

\section{TECHNICAL EXPERTISE}

- Expertise in micro/nano-scale device fabrication processes

- Wafer cleaning.

- Lithography: Optical lithography.

- Etching: Wet etching (experience in handling HF, Nano-strip).

- Thin film Deposition: RF/DC sputter, Thermal / E-beam evaporation, Spin coating of Photo-voltaic polymers.

- Wire Bonding: Ball bonder.

- 2+ years of experience with Chemical Vapor Deposition (CVD): synthesis of Single wall carbon nanotubes and 1+ years of experience with CVD growth of graphene on $\mathrm{Ni}$ and $\mathrm{Cu}$ films.

- Material characterization: 6+ years of experience with IPCE, SEM, AFM, XRD, FTIR, UV-Vis NIR, Raman Spectroscopy Capacitive Photocurrent Spectroscopy (CPS). 
- Experienced in operating Spectra Physics femto-second pulsed Laser; Hurricane (MaiTai, Evolution, Regenerative Amplifier) pumped OPA-800 CF.

- Experienced in troubleshooting of equipment problems and Labview programs for data acquisition.

- Software Packages: LabView, Matlab, L-edit, DesignCad, IGOR, Peakfit, Origin.

\section{RESEARCH EXPERIENCE}

\section{Organic Photo-voltaic (Solar Cell)}

- First experimental evidence of the variation of photocurrent spectra with thickness in organic solar cell materials.

- Theoretical model provides the charge collection length and recombination lengths of bulk hetero junction solar cells.

- Developing the Capacitive photocurrent technique to investigate the charge dissociation efficiency of organic photovoltaics.

- Thermoelectric Studies

- Experimental evidence of change of conductivity in Single-walled carbon nanotube (SWNT) and other carbon structures due to ambient gas exposure.

- Use the knowledge of Design of Experiments principles (DOE) to optimize the major variables in solar cell device fabrication to enhance the yield from $25 \%$ to $80 \%$.

- Preventive Maintenance, training and have the primary responsibility of Torr international EB-4P E-beam evaporation system.

- Troubleshooting of equipment problems and LabVIEW programs for data acquisition.

- Experience in handling Spectra Physics femto-second pulsed Laser system; Hurricane (MaiTai, Evolution, Regenerative Amplifier) pumped OPA-800 CF.

\section{Device Physics \& Electron Transport}

- Electrical characterization of carbon devices (under degassing and in-situ conditions) Single-walled carbon nanotubes and other carbon materials.

- Thermoelectric Characterization of Single-walled carbon nanotubes under various environments.

- CVD synthesis of Graphene and Single wall carbon nanotubes on $\mathrm{Si} / \mathrm{SiO} 2$ and quartz substrates. 


\section{PEER REVIEWED JOURNAL PAPERS}

- B. Abeyweera, B.W. Alphenaar, "Thickness Dependent Red Shift of the Photocurrent in Bulk Heterojunction Solar Cells", Appl. Phy. Lett., 102,041113(2013).

- B. Abeyweera, B.W. Alphenaar, "Variation of the Photocurrent Spectra due to Energy Dependent Hole Mobility in Organic Bulk Hetero-junction Solar Cells", Proceedings of Material Research Society, Vol.1435, Spring 2012.

- H. Shah, H. Liu, J. Liu, K. Fernando, A. Carver, S. Kolli, B. Abeyweera, and B. Alphenaar, "Detection of Dark States in Organic Materials through Optically Generated Electron Collisions", under review, Journal of Physical Chemistry, 2012.

- B. Abeyweera, B.W. Alphenaar, Vol. 8830, 88301O-10, Proceedings of SPIE Optics \& Photonics Conference, 2013.

- V. Chakrapani, B. Abeyweera, G. U. Sumanasekera, Andriy Sherehiy, J. C. Angus, "Electrochemically Induced p-Type Conductivity in Carbon Nanotubes", ECS Solid State Letters, 2 (11) M57-M60 (2013).

- H. Shah, H. Liu, J. Liu, K. Fernando, A. Carver, S. Kolli, B. Abeyweera, and B. Alphenaar, "Photo-induced charge transfer across an organic / contact interface: polaronic state spectroscopy", Vol 8811, Proc. SPIE 8811, Phys. Chem. of Interfaces and Nanomaterials 2013.

\section{CONFERENCE PRESENTATIONS}

- B. Abeyweera, B.W. Alphenaar, "Variation of the Photocurrent Spectra due to Energy Dependent Hole Mobility in Organic Bulk Hetero-junction Solar Cells", presented at Material Research Society, San Francisco, CA, April 2012Spring 2012.

- B. Abeyweera, B.W. Alphenaar, "Understanding the thickness dependent Photocurrent spectra of organic bulk hetero junction Solar cells" presented at the International Society for Optics and Photonics, (SPIE) San Diego, CA, August 2013.

- H. Shah, H. Liu, J. Liu, K. Fernando, A. Carver, S. Kolli, B. Abeyweera, and B. Alphenaar, "Photo-induced charge transfer across an organic / contact interface: polaronic state spectroscopy" presented at Nanomaterials \& Engineering of the International Society for Optics and Photonics (SPIE), San Diego, CA, August 2013. 
- B. Abeyweera, S.Desai \& G. Sumanasekara "Electrochemically mediated charge transfer effects in Single Walled Carbon Nanotubes (SWCNTS)" presented at the American Physical Society March meeting, Pittsburgh, Pennsylvania 2009.

- B. Abeyweera \& B. W. Alphenaar "Variation of the Photocurrent Spectra due to Energy Dependent Hole Mobility in Organic Bulk Hetero-junction Solar Cells", presented at 17th Annual Kentucky EPSCoR Conference, Lexington, KY May 2012.

- B. Abeyweera \& B. W. Alphenaar "Use of Photobleaching technique to understand the effect of photovoltage on the stability \& efficiency of organic solar cells" presented at 16th Annual Kentucky EPSCoR Conference, Louisville, KY May 2011.

- B. K. Abeyweera, H. Shah, T. Bansal, K. Medepalli \& B. W. Alphenaar "Fabrication techniques for high efficient organic polymer solar cell" presented at Engineers Day, University of Louisville, 2010.

- B. Abeyweera, S.Desai \& G. Sumanasekara "Electrochemically mediated charge transfer effects on Single-walled carbon nanotubes" presented at Graduate Student Symposium, University of Louisville, 2009.

\section{PROFESSIONAL AFFLIATIONS}

- Student member of the International Society for Optics and Photonics (SPIE)

- Student member of the Materials Research Society (MRS)

- Student member of the American Physical Society (APS)

\section{ACHIEVEMENTS AND AWARDS}

- Research Assistantship, August 2011 - Present.

- Grosscurth Fellowship, Electrical \& Computer Engineering (August 2009- July 2011)

- $\quad$ Teaching Assistantship, August 2007 - August 2009. 